\title{
Weak Lewis Pairs as Catalysts for Highly Isoselective Ring-Opening Polymerization of Epimerically Labile $\mathrm{rac}-\mathrm{O}$-Carboxyanhydride of Mandelic Acid
}

\author{
Jinxing Jiang, ${ }^{a}$ Yaqin Cui, ${ }^{a}$ Yaguang Lu, ${ }^{\mathrm{b}}$ Bin Zhang, ${ }^{\mathrm{b}}$ Xiaobo Pan, ${ }^{\mathrm{a}}$ Jincai $\mathrm{Wu}^{* \mathrm{a}}$
}

aState Key Laboratory of Applied Organic Chemistry, College of Chemistry and Chemical

Engineering, Lanzhou University, Lanzhou 730000 (P.R. China). ${ }^{b}$ School of Materials Science and Engineering, Zhengzhou University, Zhengzhou, 450002, China.

*Correspondence to:wujc@1zu.edu.cn.

This PDF file includes:

Experimental details

Figures. S1 to S57

Tables S1 to S8

Scheme S1

References for SI reference citations 


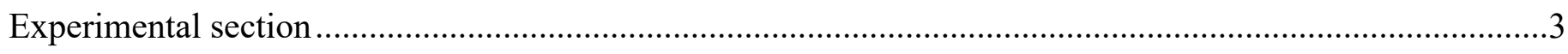

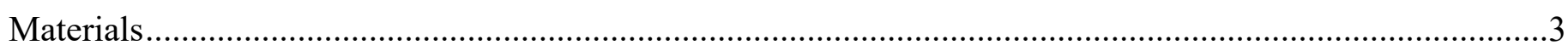

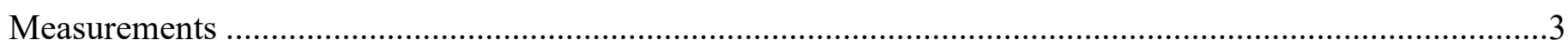

General procedure for polymerization of man OCA by Lewis pairs. ...................................................6

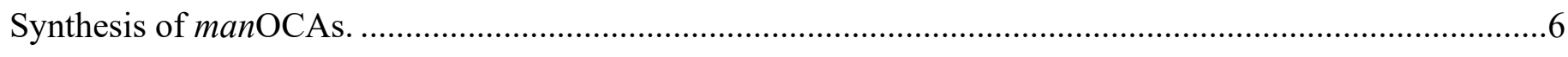

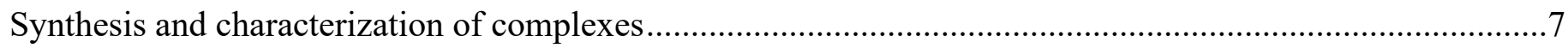

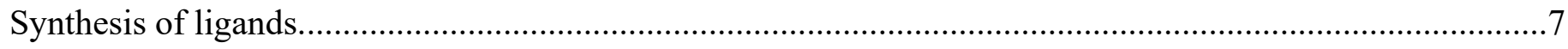

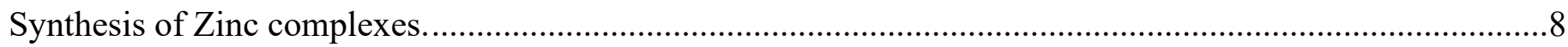

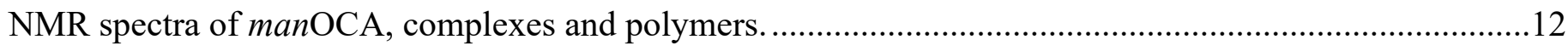

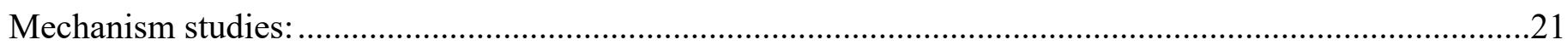

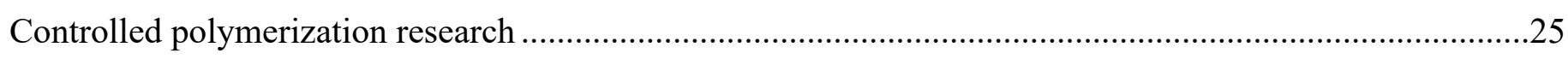

Analysis of the stereoselectivies for the ROP rac-manOCA via the weak Lewis pairs ...............................32

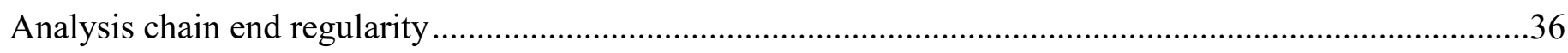

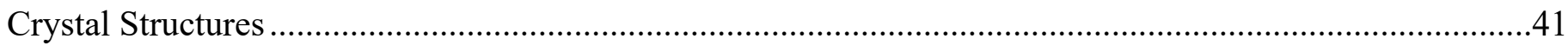

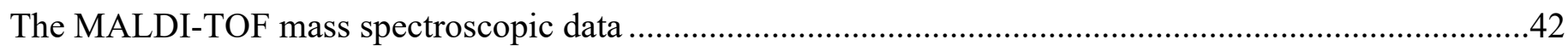

Differential scanning calorimetry (DSC) and thermal gravimetric (TG) ..............................................43

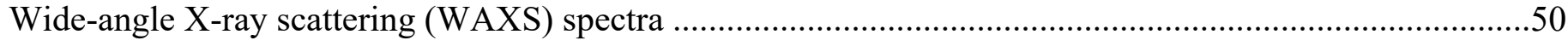

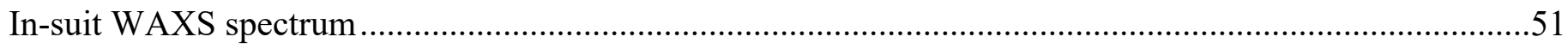

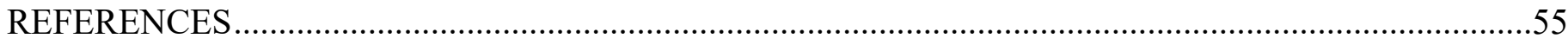




\section{Experimental section}

\section{Materials}

Toluene, THF, and n-hexane were dried by refluxing with sodium and benzophenone, the latter serve as an indicator. $\mathrm{CH}_{2} \mathrm{Cl}_{2}$ was distilled from $\mathrm{P}_{2} \mathrm{O}_{5}, \mathrm{CDCl}_{3}$, toluene- $d_{8}$ were purchased from J\&K Scientific, Ltd. in Beijing and were dried over activated molecular sieves. $\mathrm{Zn}\left[\mathrm{N}\left(\mathrm{SiMe}_{3}\right)_{2}\right]_{2}$ were synthesized according to literature procedures. ${ }^{1-2}$ D-mandelic acid, $\mathrm{L}^{-}$-mandelic acid and rac-mandelic acid were purchased from Energy Chemical and used as received, triphosgene were purchased from Beijing HWRK Chem Co. LTD. $\mathrm{D}^{-m a n O C A}, \mathrm{~L}^{-m a n O C A}$ and rac-manOCA, were synthesized according to the previous literature ${ }^{3}$. All the other employed chemicals were purchased from the local reagent company, and used as received without further purification unless otherwise stated.

\section{Measurements}

${ }^{1} \mathrm{H}$ NMR and ${ }^{13} \mathrm{C}$ NMR were recorded on Varian Mercury Plus $300 \mathrm{MHz}$ spectrometer, JNM-ECS 400 MHz and Varian INOVA $600 \mathrm{MHz} .{ }^{1} \mathrm{H}$ NMR chemical shifts are reported in ppm versus residual protons in deuterated solvents as follows: $\delta 7.26$ ppm for chloroform- $d$ and $\delta 2.08$ ppm for toluene- $d_{8} \cdot{ }^{13} \mathrm{C}$ NMR chemical shifts are reported in ppm versus residual ${ }^{13} \mathrm{C}$ in the solvents: $\delta 77.0 \mathrm{ppm}$ for chloroform- $d$.

The molecular weights $\left(M_{\mathrm{n}}\right.$ and $\left.M_{\mathrm{w}}\right)$ and the molecular mass distributions $\left(M_{\mathrm{w}} / M_{\mathrm{n}}\right)$ of the polymer samples were measured by gel permeation chromatography (GPC) at $40{ }^{\circ} \mathrm{C}$ using $\mathrm{THF}$ as an eluent, an eluent flow rate of $1.0 \mathrm{~mL} / \mathrm{min}$, and narrow polystyrene standards as reference samples. The measurements were performed using a Shodex GPC KF-800 system that was equipped with a Shodex RI-201H detector, using Shodex-KF-803 and shodex-KF-804 connected Styragel columns (400 - $400000 \mathrm{~kg} / \mathrm{mol})$. Poly(manOCA) is not corrected.

Matrix-Assisted Laser Desorption/ Ionization Time of Flight (MALDI-TOF) Mass Spectrometry conditions were as follows. Instrument type: Shimadzu MALDI-7090, Tuning Reflectron, Power 25, P.Ext at 5000.00 
(bin 353), Ion Gate Blanking: 400.00, Laser Diameter: 100. The MALDI-TOF mass spectroscopic data were obtained using trans-2-[3-(4-tert-butylphenyl)-2-methyl-2-propenylidene]malonitrile (DCTB) as the matrix (10 $\mathrm{mgmL}^{-1}$ in THF), and sodium trifluoroacetate as the cationization agent $\left(10 \mathrm{mg} / \mathrm{mL}^{-1}\right.$ in THF). Samples were dissolved in THF $\left(10 \mathrm{mg} / \mathrm{mL}^{-1}\right)$. Solutions of matrix and salt were mixed in a volume ratio of 4: 1 . Then the mixed solution $(0.5 \mu \mathrm{L})$, samples $(0.5 \mu \mathrm{L})$ and mixed solution $(0.5 \mu \mathrm{L})$ was hand-spotted on a stainless steel MALDI target in order, which allowed to be dried completely.

X-ray diffraction measurements were performed on a SuperNova (Dual) X-ray diffraction diffractometer with a graphite-monochromated $\mathrm{Cu} \mathrm{K} \alpha$ radiation source $(\lambda=1.54184 \AA)$. The structures were solved by direct methods using the Siemens SHELXTL PLUS program. ${ }^{4}$ Non-hydrogen atoms were refined with anisotropic displacement parameters during the final cycles. All hydrogen atoms were placed by geometrical considerations and were added to the structure-factor calculations.

Differential scanning calorimetry (DSC) measurements, using 5-10 $\mathrm{mg}$ of material, were performed on a DSC $2500 \mathrm{TA}$ instrument in using a heat $\left(25-220^{\circ} \mathrm{C}\right) / \operatorname{cool}\left(220-25^{\circ} \mathrm{C}\right) /$ heat $\left(25-220^{\circ} \mathrm{C}\right)$ cycle at a rate of $10{ }^{\circ} \mathrm{C} \mathrm{min}^{-1}$ under a $\mathrm{N}_{2}$ atmosphere. (the data of Figure S49-51 were measured by NETZSCH STA 449F3 instrument)

Thermal gravimetric analyzer: using 5-10 mg of material, were performed on a NETZSCH STA 449F3 instrument in using a heat $\left(40-400^{\circ} \mathrm{C}\right)$ at a rate of $10{ }^{\circ} \mathrm{C}$ min- -1 under a $\mathrm{N}_{2}$ atmosphere.

Method for preparing DSC and TGA samples. These samples were prepared by the following method: about $40 \mathrm{mg}$ poly $(\operatorname{manOCA})\left(M_{\mathrm{n}} \approx 5300\right)$ was dissolved in $2 \mathrm{~mL}$ of DCM, stirred slowly for $6 \mathrm{~h}$, then $4 \mathrm{~mL}$ of methanol was added dropwise, the solution formed two phases. After stirring for several hours, a white powder was precipitated. After Stop stirring for 30 minutes, the filtrate was poured out and the sample was vacuum dried. The obtained polymers were vacuum dried under a $\mathrm{N}_{2}$ atmosphere at a specific temperature for 3 hours in order to eliminate the influence of the solvent: poly $\left.{ }_{L}-\operatorname{manOCA}\right)(5.3 \mathrm{~kg} / \mathrm{mol}, Ð=1.10$; Table 


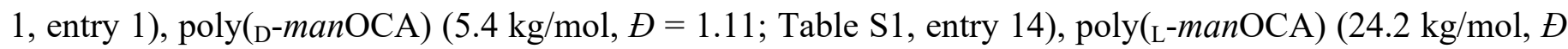

$=1.13 ;$ Table 1 , entry 6$)$ and poly $($ rac-manOCA $)(1: 1$ mixture enantiopure poly $(\mathrm{L}-m a n \mathrm{OCA})$ and $\left.\operatorname{poly}\left(\mathrm{D}^{-m a n O C A}\right)\right)(5.3 \mathrm{~kg} / \mathrm{mol}, Ð=1.11)$ were heated at $130{ }^{\circ} \mathrm{C}$, poly $(\mathrm{rac}-m a n \mathrm{OCA})\left(P_{\mathrm{m}}=0.6,5.4 \mathrm{~kg} / \mathrm{mol}\right.$, $Ð=1.10$; Table 2, entry 1$)$ and poly(rac-manOCA $)\left(P_{\mathrm{m}}=0.92,5.3 \mathrm{~kg} / \mathrm{mol}, Ð=1.12\right.$; Table 2, entry 4) were heated at $90^{\circ} \mathrm{C}$.

X-ray characterization

In-suit WAXS measurement are performed by a Nano-inXider vertical system (Xenocs, Sassenage, France) equipped with two semiconductor detectors (Pilatus $100 \mathrm{~K}$ for WAXS and Pilatus 200K for SAXS, DECTRIS, Swiss). The sample-to-detector distances are $79 \mathrm{~nm}$. A multilayer focused the $\mathrm{Cu} \mathrm{K} \alpha \mathrm{X}$-ray source (the X-ray wavelength is $0.154 \mathrm{~nm}, \mathrm{Genix}^{3 \mathrm{D}} \mathrm{Cu}$ ULD, Xenocs SA, France) is generated at $50 \mathrm{kv}$ and $0.6 \mathrm{~mA}$. The evacuated chamber is achieved by using a vacuum pump to reduce air scatting and absorption. The data are normalized with respect to the primary beam intensity and corrected for background scatting. 


\section{General procedure for polymerization of manOCA by Lewis pairs.}

A typical polymerization procedure is illustrated by the synthesis of Poly $\left(\mathrm{L}_{\mathrm{L}}-\mathrm{man} \mathrm{OCA}\right)$ (Table 1 , entry 1). $\mathrm{L}_{\text {-manOCA }}(0.089 \mathrm{~g}, 0.5 \mathrm{mmol})$ was added into a $2 \mathrm{~mL}$ toluene solution of $0.005 \mathrm{mmol}$ of 1, $0.01 \mathrm{mmol}$ 3-fluoropyridine (3-F-Py), $0.01 \mathrm{mmol}$ methyl lactate (ML). The solution was rapidly stirred at room temperature for $30 \mathrm{~min}$. An aliquot was withdrawn to determine monomer conversion by ${ }^{1} \mathrm{H}$ NMR spectroscopy. The polymer was precipitated via adding methanol $(2 \mathrm{~mL})$. A white solid was obtained by recrystallization from a $\mathrm{CH}_{2} \mathrm{Cl}_{2} /$ methanol mixed solvent (if the mixed solvent of n-hexane/methanol was used, no melting point was found in DSC experiments) and dried under vacuum.

Poly(L-mandelic acid): ${ }^{1} \mathrm{H}$ NMR (400 MHz, $\left.\mathrm{CDCl}_{3}\right): \delta 7.14-7.40(\mathrm{~m}, 5 \mathrm{H}), 5.94(\mathrm{~s}, 1 \mathrm{H}) .{ }^{13} \mathrm{C}$ NMR $\left(100 \mathrm{MHz}, \mathrm{CDCl}_{3}\right): \delta 166.75,132.25,129.35,128.64,127.79,74.70$.

\section{Synthesis of manOCAs.}

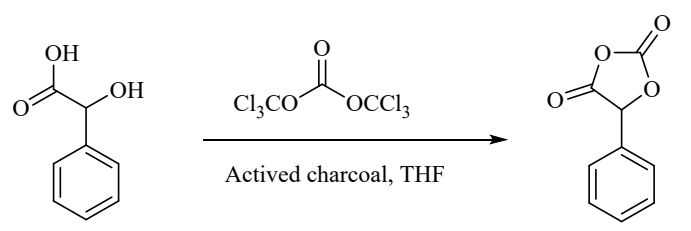

According to literature procedures. ${ }^{3}$ To a solution of mandelic acid $(100.0 \mathrm{mmol}, 15.2 \mathrm{~g})$ and activated charcoal $(\sim 1.3 \mathrm{~g})$ in anhydrous THF $(100 \mathrm{~mL})$, a solution of triphosgene $(90.0 \mathrm{mmol}, 26.7$ g) in $50 \mathrm{~mL}$ THF was added at $0{ }^{\circ} \mathrm{C}$. The reaction mixture was stirred for 12 hours at room temperature. The mixture was filtered through celite, the filtrate was removed under reduced pressure and trapped with liquid nitrogen. *** CAUTION: The latter solution was carefully neutralized at $0{ }^{\circ} \mathrm{C}$ with aqueous ammoniac. $* * *$ Recrystallization from dry DCM/n-hexane at -30 ${ }^{\circ} \mathrm{C}$ three times afforded colorless crystals. ${ }^{1} \mathrm{H} \mathrm{NMR}\left(\mathrm{CDCl}_{3}, 400 \mathrm{MHz}\right): \delta 6.02(\mathrm{~s}, 1 \mathrm{H}), 7.40-7.46(\mathrm{~m}$, $5 \mathrm{H}) ;{ }^{13} \mathrm{C} \mathrm{NMR}\left(100 \mathrm{MHz}, \mathrm{CDCl}_{3}\right): \delta 80.40,126.11,129.22,129.53,130.78,147.96,165.28$; 
rac-manOCA: yield, $67 \%,[\alpha]_{\mathrm{D}}^{20.0}=0.0\left(10 \mathrm{mg} / \mathrm{mL}, \mathrm{CHCl}_{3}\right)$.

L-manOCA: yield, $78 \%,[\alpha]_{\mathrm{D}}^{20.0}=+20.0\left(10 \mathrm{mg} / \mathrm{mL}, \mathrm{CHCl}_{3}\right)$.

D-manOCA: yield, $76 \%,[\alpha]_{\mathrm{D}}^{20.0}=-20.0\left(10 \mathrm{mg} / \mathrm{mL}, \mathrm{CHCl}_{3}\right)$.

\section{Synthesis and characterization of complexes}

\section{Synthesis of ligands.}

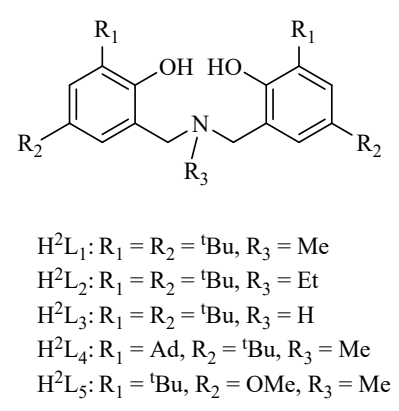

$\mathrm{H}^{2} \mathrm{~L}_{1}$ and $\mathrm{H}^{2} \mathrm{~L}_{4}$ were synthesized according to literature procedures, and the data of NMR is consistent with the literature. ${ }^{5}$

$\mathrm{H}^{2} \mathrm{~L}_{1}:{ }^{1} \mathrm{H}$ NMR (400 MHz, Chloroform-d) $\delta 7.99(\mathrm{~s}, 2 \mathrm{H}, \mathrm{OH}), 7.24(\mathrm{~d}, J=2.4 \mathrm{~Hz}, 2 \mathrm{H}, \mathrm{Ph} H), 6.92$ (d, $J=2.4 \mathrm{~Hz}, 2 \mathrm{H}, \mathrm{PhH}), 3.64\left(\mathrm{~s}, 4 \mathrm{H}, \mathrm{CH}_{2}\right), 2.33\left(\mathrm{~s}, 3 \mathrm{H}, \mathrm{CH}_{3}\right), 1.41\left(\mathrm{~s}, 18 \mathrm{H},{ }^{t} \mathrm{Bu}\right), 1.28\left(\mathrm{~s}, 18 \mathrm{H},{ }^{t} \mathrm{Bu}\right)$. ${ }^{13} \mathrm{C}$ NMR (100 MHz, Chloroform- $d$ ) $\delta 152.42,141.51,136.01,124.92,123.57,121.54,59.98,42.07$, $34.85,34.16,31.63,29.69$.

$\mathrm{H}^{2} \mathrm{~L}_{4}:{ }^{1} \mathrm{H}$ NMR (400 MHz, Chloroform- $d$ ) $\delta 8.20(\mathrm{~s}, 2 \mathrm{H}, \mathrm{OH}), 7.18(\mathrm{~d}, J=2.3 \mathrm{~Hz}, 2 \mathrm{H}, \mathrm{Ph} H), 6.92$ (d, $J=2.3 \mathrm{~Hz}, 2 \mathrm{H}, \mathrm{PhH}), 3.63\left(\mathrm{~s}, 4 \mathrm{H}, \mathrm{CH}_{2}\right), 2.36$ (s, 3H, $\left.\mathrm{CH}_{3}\right), 2.15$ (s, 12H, $\mathrm{CH}_{2}$-adamantyl), 2.06 (bs, 6H, CH-adamantyl), 1.77 (s, 12H, $\mathrm{CH}_{2}$-adamantyl), 1.29 (s, $\left.18 \mathrm{H},{ }^{t} \mathrm{Bu}\right) .{ }^{13} \mathrm{C} \mathrm{NMR}(100 \mathrm{MHz}$, Chloroform-d) $\delta 152.64,141.66,136.48,124.79,123.51,121.76,59.60,42.14,40.55,37.15,34.23$, $31.64,29.18$. 
$\mathrm{H}^{2} \mathrm{~L}_{2}$ was synthesized according to literature procedures. ${ }^{6}$ The data of NMR is consistent with the literature. ${ }^{1} \mathrm{H}$ NMR (300 MHz, Chloroform- $d$ ) $\delta 8.02(\mathrm{~s}, 2 \mathrm{H}, \mathrm{OH}), 7.23(\mathrm{~d}, J=2.5 \mathrm{~Hz}, 2 \mathrm{H}, \mathrm{Ph} H)$, $6.93(\mathrm{~d}, J=2.4 \mathrm{~Hz}, 2 \mathrm{H}, \mathrm{PhH}), 3.70(\mathrm{~s}, 4 \mathrm{H}, \mathrm{CH}), 2.65\left(\mathrm{q}, J=7.2 \mathrm{~Hz}, 2 \mathrm{H}, \mathrm{CH}_{2} \mathrm{CH}_{3}\right), 1.41(\mathrm{~s}, 18 \mathrm{H}$, $\left.{ }^{t} \mathrm{Bu}\right), 1.28\left(\mathrm{~s}, 18 \mathrm{H}, \quad{ }^{t} \mathrm{Bu}\right), 1.19\left(\mathrm{t}, J=7.2 \mathrm{~Hz}, 3 \mathrm{H}, \mathrm{CH}_{2} \mathrm{CH}_{3}\right) \cdot{ }^{13} \mathrm{C}$ NMR $(75 \mathrm{MHz}$, Chloroform- $d) \delta$ $152.42,141.45,135.98,125.00,123.48,121.58,56.54,47.16,34.84,34.15,31.63,29.66,11.13$.

$\mathrm{H}^{2} \mathrm{~L}_{3}$ was synthesized according to literature procedures. ${ }^{7-8}$ The data of NMR is consistent with the literature. ${ }^{1} \mathrm{H}$ NMR (400 MHz, Chloroform- $d$ ) $\delta 7.25(\mathrm{~d}, J=2.4 \mathrm{~Hz}, 2 \mathrm{H}, \mathrm{Ph} H), 6.96(\mathrm{~d}, J=2.4 \mathrm{~Hz}$, $2 \mathrm{H}, \mathrm{PhH}), 3.92\left(\mathrm{~s}, 4 \mathrm{H}, \mathrm{CH}_{2}\right), 1.43\left(\mathrm{~s}, 18 \mathrm{H},{ }^{t} \mathrm{Bu}\right), 1.29\left(\mathrm{~s}, 18 \mathrm{H},{ }^{t} \mathrm{Bu}\right) .{ }^{13} \mathrm{C} \mathrm{NMR}(100 \mathrm{MHz}$, Chloroform- $d$ ) $\delta 152.64,141.64,135.88,124.39,123.46,122.81,51.44,34.79,34.21,31.65,29.84$.

$\mathrm{H}^{2} \mathrm{~L}_{5}: \mathrm{H}^{2} \mathrm{~L}_{5}$ was prepared via the same method for synthesis of $\mathrm{H}^{2} \mathrm{~L}_{1}$. Methylamine $(25-30 \% \mathrm{w} / \mathrm{w}$ solution in $\mathrm{H}_{2} \mathrm{O}, 1.24 \mathrm{~g}, 10.0 \mathrm{mmol}$ ) was added dropwise to a stirring solution of paraformaldehyde (0.6 g, $10.0 \mathrm{mmol})$ and 2-methoxy-4-tert-butylphenol $(3.6 \mathrm{~g}, 20.0 \mathrm{mmol})$ in a mixed solvent of 50 $\mathrm{mL}$ methanol and $50 \mathrm{~mL} \mathrm{H}_{2} \mathrm{O}$. And the reaction was refluxed for $12 \mathrm{~h}$. After the reaction was cooled, the product was obtained as a separate phase which could be easily isolated. And the crude product recrystallized from methanol to afford a white powder $\mathrm{H}^{2} \mathrm{~L}_{4}$, yield $(55 \%, 2.3 \mathrm{~g}) .{ }^{1} \mathrm{H} \mathrm{NMR}(400 \mathrm{MHz}$, Chloroform-d) $\delta 7.76(\mathrm{~s}, 2 \mathrm{H}, \mathrm{OH}), 6.83(\mathrm{~d}, \mathrm{~J}=3.1 \mathrm{~Hz}, 2 \mathrm{H}, \mathrm{Ph} H), 6.50(\mathrm{~d}, \mathrm{~J}=3.0 \mathrm{~Hz}, 2 \mathrm{H}, \mathrm{Ph} H), 3.76$ $\left(\mathrm{s}, 6 \mathrm{H}, \mathrm{OCH}_{3}\right), 3.62\left(\mathrm{~s}, 4 \mathrm{H}, \mathrm{CH}_{2}\right), 2.32\left(\mathrm{~s}, 3 \mathrm{H}, \mathrm{CH}_{3}\right), 1.40\left(\mathrm{~s}, 18 \mathrm{H},{ }^{\mathrm{t}} \mathrm{Bu}\right) .{ }^{13} \mathrm{C} \mathrm{NMR}(100 \mathrm{MHz}$, Chloroform- $d$ ) $\delta 152.31,148.71,138.50,122.98,113.33,112.36,59.82,55.63,41.98,34.85,29.53$.

\section{Synthesis of Zinc complexes.}



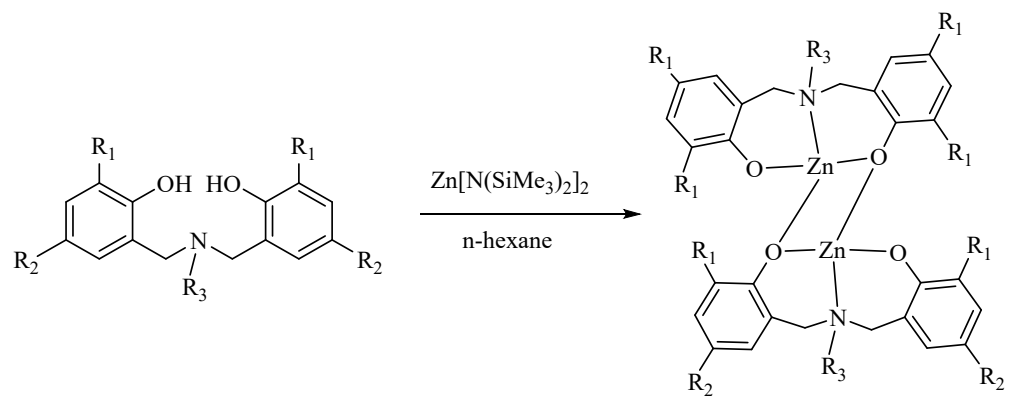

$$
\begin{array}{ll}
1 & \mathrm{R}_{1}=\mathrm{R}_{2}={ }^{t} \mathrm{Bu}, \mathrm{R}_{3}=\mathrm{Me} \\
2 & \mathrm{R}_{1}=\mathrm{R}_{2}={ }^{t} \mathrm{Bu}, \mathrm{R}_{3}=\mathrm{Et} \\
\text { 3 } \mathrm{R}_{1}=\mathrm{R}_{2}={ }^{t} \mathrm{Bu}, \mathrm{R}_{3}=\mathrm{H} \\
4 \mathrm{R}_{1}=\mathrm{Ad}, \mathrm{R}_{2}={ }^{t} \mathrm{Bu}, \mathrm{R}_{3}=\mathrm{Me} \\
5 \mathrm{R}_{1}={ }^{t} \mathrm{Bu}, \mathrm{R}_{2}=\mathrm{OMe}, \mathrm{R}_{3}=\mathrm{Me}
\end{array}
$$

Synthesis of complex 1.
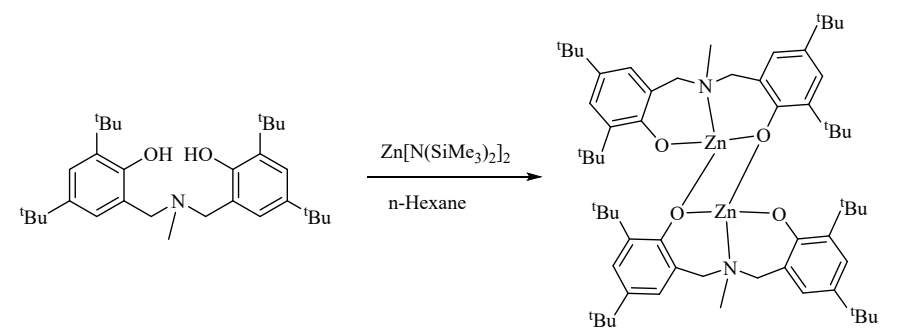

Complexes 1 were prepared via procedures in literature. ${ }^{5}$

$\mathrm{Zn}\left[\mathrm{N}\left(\mathrm{SiMe}_{3}\right)_{2}\right]_{2}(0.386 \mathrm{~g}, 1.0 \mathrm{mmol})$ was added into a solution of $\mathrm{H}^{2} \mathrm{~L}_{1}(0.467 \mathrm{~g}, 1.0 \mathrm{mmol})$ in $10 \mathrm{~mL}$ n-hexane. After stirring the mixture for $12 \mathrm{~h}$, a large amount of white solid precipitate formed and was filtered through celite on fritted glass. After washing with n-hexane twice and drying under vacuum for $6 \mathrm{~h}, 0.3 \mathrm{~g}$ white powder solid was obtained with a yield of $70 \%$. Single crystals of $\mathbf{L}^{1} \mathbf{Z n}$ + 3-F-Py were obtained from a mixture of 1/3-F-Py (1/2) in a saturated mixed solution of toluene and n-hexane at room temperature. ${ }^{1} \mathrm{H}$ NMR (400 MHz, Chloroform- $\left.d\right) \delta 7.24(\mathrm{~d}, J=2.5 \mathrm{~Hz}, 2 \mathrm{H}$, $\mathrm{Ph} H), 7.12(\mathrm{~d}, J=2.5 \mathrm{~Hz}, 2 \mathrm{H}, \mathrm{Ph} H), 6.95(\mathrm{~d}, J=2.5 \mathrm{~Hz}, 2 \mathrm{H}, \mathrm{Ph} H), 6.69(\mathrm{~d}, J=2.5 \mathrm{~Hz}, 2 \mathrm{H}, \mathrm{Ph} H)$, $5.04\left(\mathrm{~d}, J=12.7 \mathrm{~Hz}, 2 \mathrm{H}, \mathrm{CH}_{2}\right), 3.97\left(\mathrm{~d}, J=12.7 \mathrm{~Hz}, 2 \mathrm{H}, \mathrm{CH}_{2}\right), 3.35\left(\mathrm{~d}, J=12.7 \mathrm{~Hz}, 2 \mathrm{H}, \mathrm{CH}_{2}\right), 2.61$ $\left(\mathrm{s}, 6 \mathrm{H}, \mathrm{NCH}_{3}\right), 2.54\left(\mathrm{~d}, J=12.7 \mathrm{~Hz}, 2 \mathrm{H}, \mathrm{CH}_{2}\right), 1.39\left(\mathrm{~s}, 18 \mathrm{H},{ }^{t} \mathrm{Bu}\right), 1.27\left(\mathrm{~s}, 18 \mathrm{H},{ }^{t} \mathrm{Bu}\right), 1.21(\mathrm{~s}, 36 \mathrm{H}$, $\left.{ }^{t} \mathrm{Bu}\right) .{ }^{13} \mathrm{C}$ NMR (100 MHz, Chloroform- $\left.d\right) \delta 161.86,156.84,141.82,138.30,137.84,135.98,126.93$, 
$31.76,31.53,31.09,29.39$.

Synthesis of complex $2+3-F-P y$.
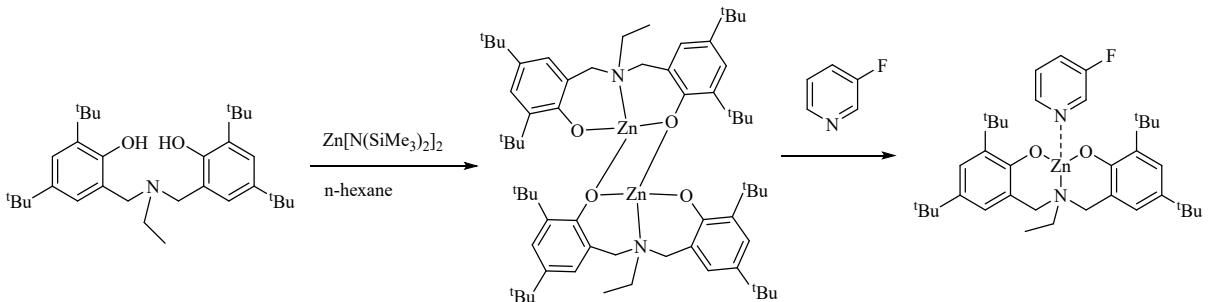

The synthesis of complex $\mathbf{L}^{2} \mathbf{Z n}+3$ F-Py:

$\mathrm{Zn}\left[\mathrm{N}\left(\mathrm{SiMe}_{3}\right)_{2}\right]_{2}(0.386 \mathrm{~g}, 1.0 \mathrm{mmol})$ was added into a solution of $\mathrm{H}^{2} \mathrm{~L}_{2}(0.481 \mathrm{~g}, 1.0 \mathrm{mmol})$ in 10 $\mathrm{mL}$-hexene. After stirring the reaction mixture for $6 \mathrm{~h}, 3-\mathrm{F}-\mathrm{Py}(0.11 \mathrm{~g}, 1.1 \mathrm{mmol})$ was added into the above solution. After stirring for another $3 \mathrm{~h}$, a large amount of white solid was precipitated. The precipitate was filtered through celite on fritted glass. After washing with n-hexane twice and drying under vacuum for $6 \mathrm{~h}, 0.4 \mathrm{~g}$ white powder solid was obtained with a yield $62 \%$. Single crystal was obtained from a saturated toluene and n-hexane solution at room temperature. ${ }^{1} \mathrm{H}$ NMR $(400 \mathrm{MHz}$, Chloroform- $d$ ) $\delta 8.67(\mathrm{~s}, 1 \mathrm{H}), 8.57(\mathrm{~d}, \mathrm{~J}=4.6 \mathrm{~Hz}, 1 \mathrm{H}), 7.73(\mathrm{td}, \mathrm{J}=8.6,1.9 \mathrm{~Hz}, 1 \mathrm{H}), 7.57(\mathrm{dt}, \mathrm{J}=$ 9.0, $5.0 \mathrm{~Hz}, 1 \mathrm{H}), 7.23(\mathrm{~d}, \mathrm{~J}=2.4 \mathrm{~Hz}, 2 \mathrm{H}), 6.83(\mathrm{~d}, \mathrm{~J}=2.3 \mathrm{~Hz}, 2 \mathrm{H}), 3.86-3.56(\mathrm{~m}, 4 \mathrm{H}), 2.72(\mathrm{q}, \mathrm{J}=$ $7.1 \mathrm{~Hz}, 2 \mathrm{H}), 1.43(\mathrm{~s}, 18 \mathrm{H}), 1.26(\mathrm{~s}, 18 \mathrm{H}), 0.98(\mathrm{t}, \mathrm{J}=7.2 \mathrm{~Hz}, 3 \mathrm{H}) .{ }^{13} \mathrm{C} \mathrm{NMR}(100 \mathrm{MHz}$, Chloroform- $d$ ) $\delta 163.09,145.75,138.81,138.53,137.71,135.85,127.35,126.45,126.13,124.14$, $120.85,58.63,48.17,35.33,33.87,31.83,29.69,8.72$.

Synthesis of complex 3 .
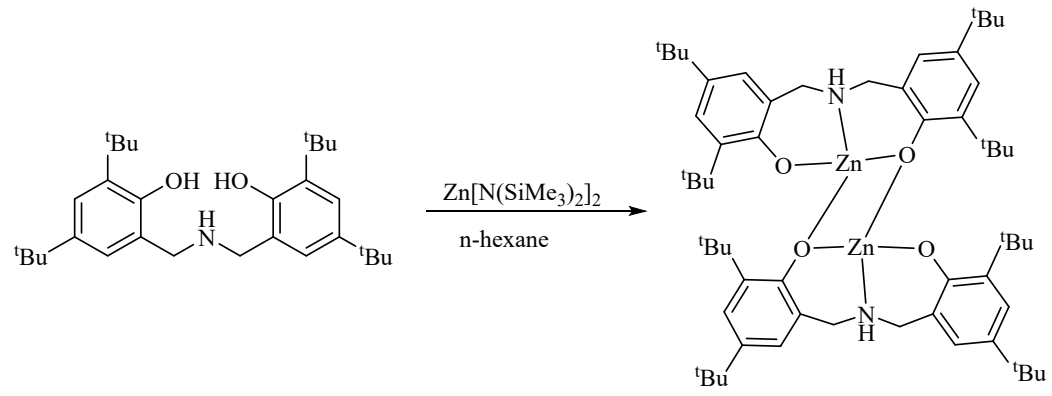

The method of synthesis of complex 3 is similar to complex 1 according reference. ${ }^{5}$ Yield $68 \% .{ }^{1} \mathrm{H}$ NMR (400 MHz, Chloroform- $d$ ) $\delta 7.18(\mathrm{~s}, 2 \mathrm{H}), 7.04(\mathrm{~s}, 2 \mathrm{H}), 6.96(\mathrm{~s}, 2 \mathrm{H}), 6.76(\mathrm{~s}, 2 \mathrm{H}), 4.74(\mathrm{~d}, \mathrm{~J}=$ $12.4 \mathrm{~Hz}, 2 \mathrm{H}), 4.02-3.86(\mathrm{~m}, 4 \mathrm{H}), 3.33(\mathrm{~d}, \mathrm{~J}=12.4 \mathrm{~Hz}, 2 \mathrm{H}), 2.75(\mathrm{~d}, \mathrm{~J}=14.5 \mathrm{~Hz}, 2 \mathrm{H}), 1.24$ (s, $36 \mathrm{H}), 1.19$ (s, 18H), 1.10 (s, 18H). ${ }^{13} \mathrm{C}$ NMR (100 MHz, Chloroform- $d$ ) $\delta 161.64,157.36,141.81$, 138.38, 138.26, 136.94, 126.70, 124.75, 124.13, 123.15, 121.96, 53.18, 51.57, 35.04, 34.89, 34.08, $33.86,31.71,31.58,30.23,29.21$.

Synthesis of complex 4. 


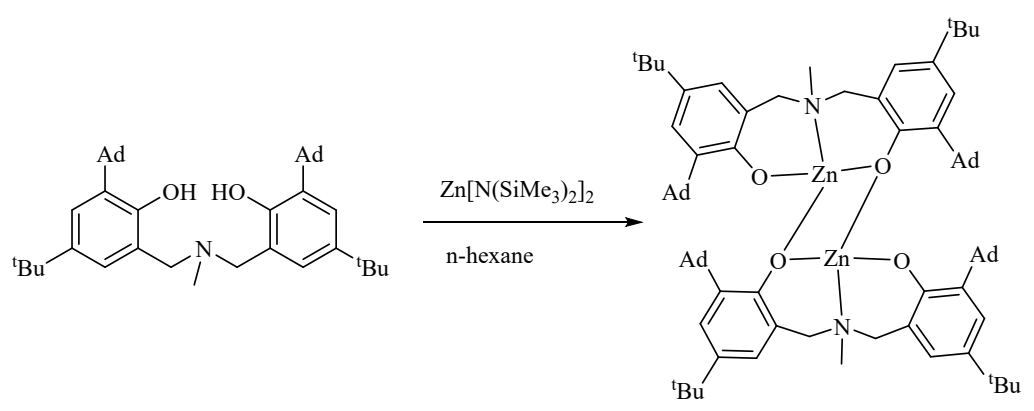

The method of synthesis of complex 4 is similar to complex 1 according reference. ${ }^{5}$ Yield $70 \% .{ }^{1} \mathrm{H}$ NMR (600 MHz, Chloroform- $d) \delta 7.28(\mathrm{~s}, 2 \mathrm{H}), 7.09(\mathrm{~s}, 2 \mathrm{H}), 6.92(\mathrm{~s}, 2 \mathrm{H}), 6.70(\mathrm{~s}, 2 \mathrm{H}), 5.21(\mathrm{~d}, J=$ $13.0 \mathrm{~Hz}, 2 \mathrm{H}), 4.05(\mathrm{~d}, J=13.0 \mathrm{~Hz}, 2 \mathrm{H}), 3.36(\mathrm{~d}, J=13.0 \mathrm{~Hz} 2 \mathrm{H}), 2.59-2.61(\mathrm{~m}, 8 \mathrm{H}), 2.19-2.03$ $(\mathrm{m}, 24 \mathrm{H}), 1.97(\mathrm{~s}, 6 \mathrm{H}), 1.78(\mathrm{~s}, 6 \mathrm{H}), 1.74-1.57(\mathrm{~m}, 24 \mathrm{H}), 1.27(\mathrm{~s}, 18 \mathrm{H}), 1.22(\mathrm{~s}, 18 \mathrm{H}) .{ }^{13} \mathrm{C} \mathrm{NMR}$ $(100 \mathrm{MHz}$, Chloroform- $d) \delta 162.59,157.68,141.51,138.31,138.02,136.57,127.00,126.33$, $125.94,124.25,124.00,119.82,65.31,60.69,45.10,42.46,40.34,38.79,37.43,37.29,36.79,34.05$, $33.85,31.75,31.59,31.52,29.28,28.79,22.66,14.14$.

Synthesis of complex $\mathbf{5}$.
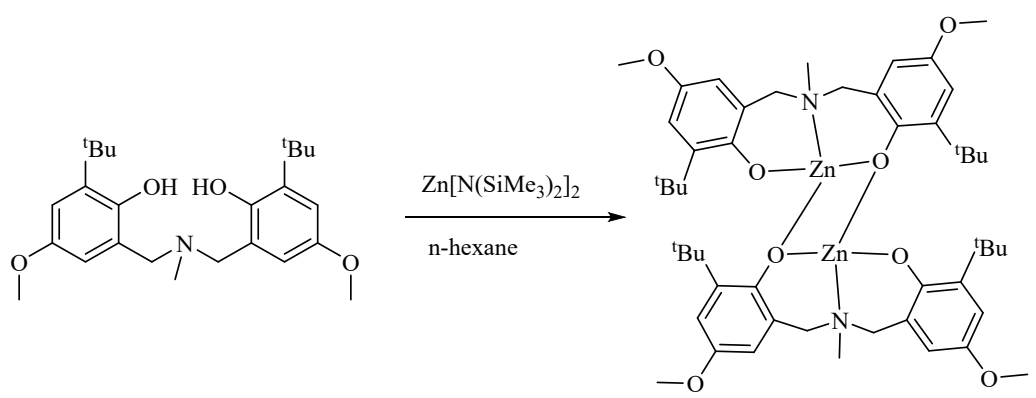

The method of synthesis of complex 5 is similar to complex $\mathbf{1}$ according reference. Yield $63 \% .{ }^{1} \mathrm{H}$ NMR (600 MHz, Chloroform- $d$ ) $\delta 6.85(\mathrm{~d}, \mathrm{~J}=3.0 \mathrm{~Hz}, 2 \mathrm{H}), 6.75(\mathrm{~d}, \mathrm{~J}=3.0 \mathrm{~Hz}, 2 \mathrm{H}), 6.56(\mathrm{~d}, \mathrm{~J}=3.0$ $\mathrm{Hz}, 2 \mathrm{H}), 6.31(\mathrm{~d}, \mathrm{~J}=3.0 \mathrm{~Hz}, 2 \mathrm{H}), 4.90(\mathrm{~d}, \mathrm{~J}=12.7 \mathrm{~Hz}, 2 \mathrm{H}), 4.01(\mathrm{~d}, \mathrm{~J}=12.7 \mathrm{~Hz}, 2 \mathrm{H}), 3.77(\mathrm{~s}, 6 \mathrm{H})$, $3.68(\mathrm{~s}, 6 \mathrm{H}), 3.31(\mathrm{~d}, \mathrm{~J}=12.7 \mathrm{~Hz}, 2 \mathrm{H}), 2.59(\mathrm{~s}, 6 \mathrm{H}), 2.53(\mathrm{~d}, \mathrm{~J}=12.7 \mathrm{~Hz}, 2 \mathrm{H}), 1.37(\mathrm{~s}, 18 \mathrm{H}), 1.17$ (s, $18 \mathrm{H}) .{ }^{13} \mathrm{C}$ NMR (100 MHz, Chloroform- $d$ ) $\delta$ 158.46, 153.25, 152.08, 148.44, 140.38, 140.02, $124.94,120.35,114.46,114.36,113.72,64.64,60.39$, 56.02, 55.68, 46.98, 35.16, 35.11, 30.86, 29.07. 
NMR spectra of manOCA, complexes and polymers.

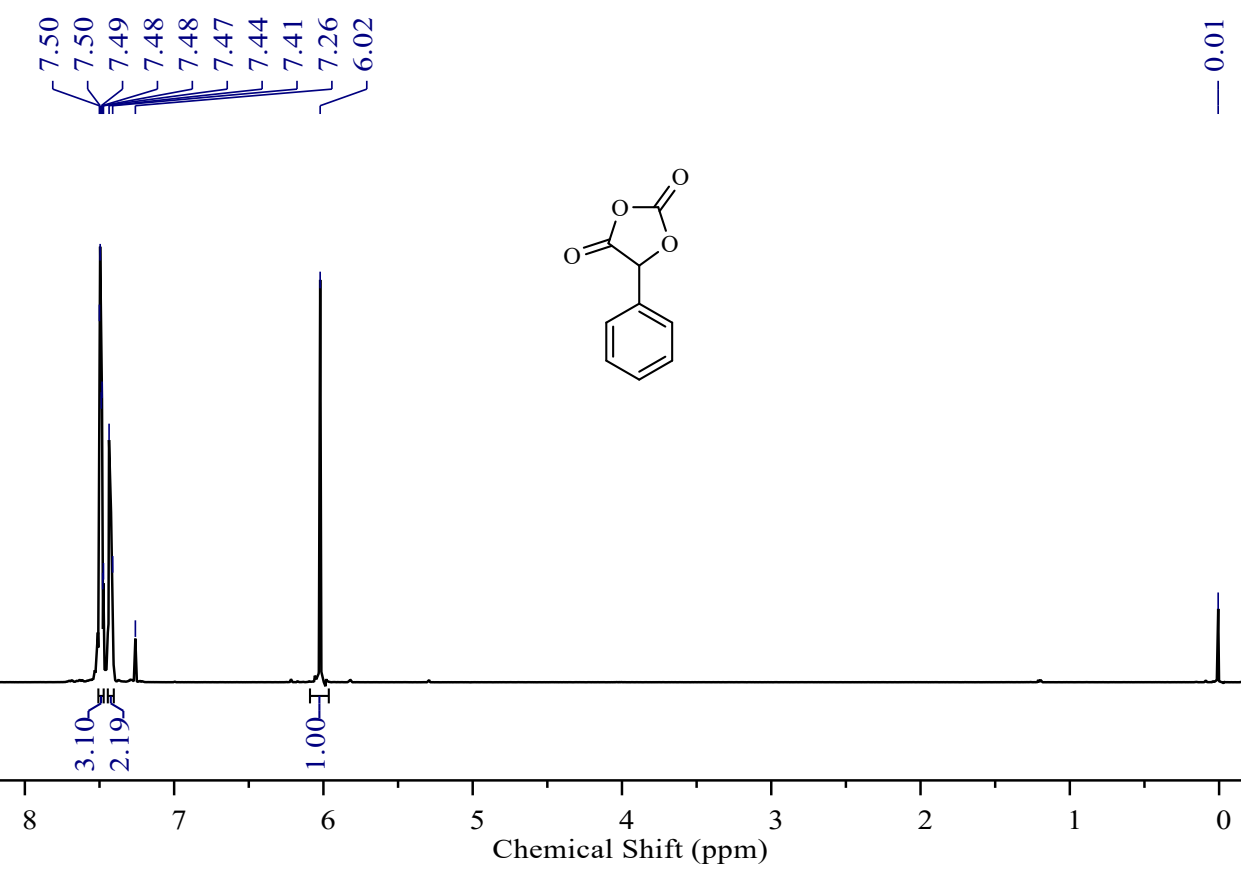

Figure $\mathrm{S} 1$. The ${ }^{1} \mathrm{H}$ NMR spectrum of man $\mathrm{OCA}\left(400 \mathrm{MHz}, \mathrm{CDCl}_{3}\right)$.

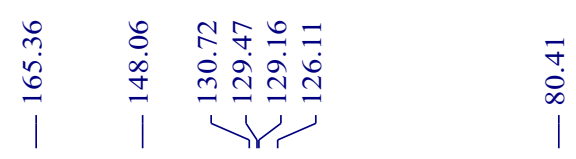

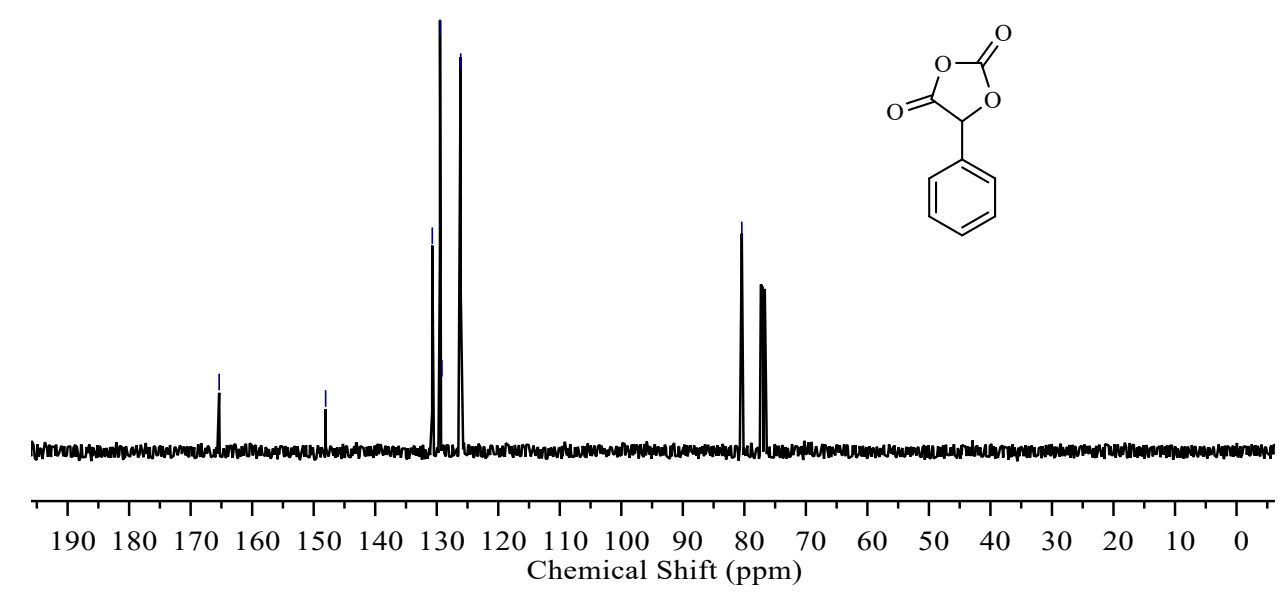

Figure S2. The ${ }^{13} \mathrm{C}$ NMR spectrum of $\operatorname{man} \mathrm{OCA}\left(100 \mathrm{MHz}, \mathrm{CDCl}_{3}\right)$. 


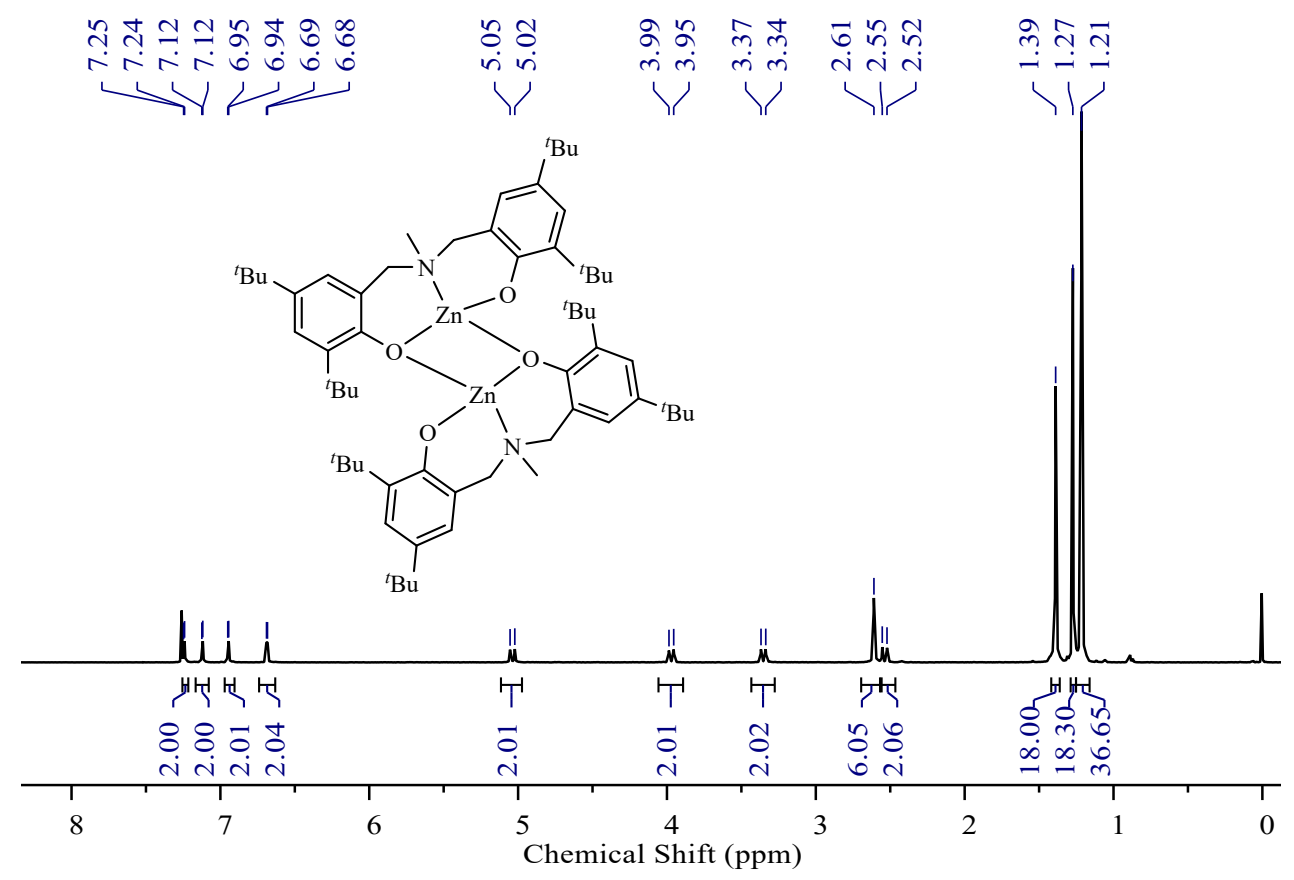

Figure S3. The ${ }^{1} \mathrm{H}$ NMR spectrum of complex $\mathbf{1}\left(\left(\mathbf{L}^{\mathbf{1}}\right)_{2} \mathbf{Z n}_{\mathbf{2}}\right)\left(\left(400 \mathrm{MHz}, \mathrm{CDCl}_{3}\right)\right.$.

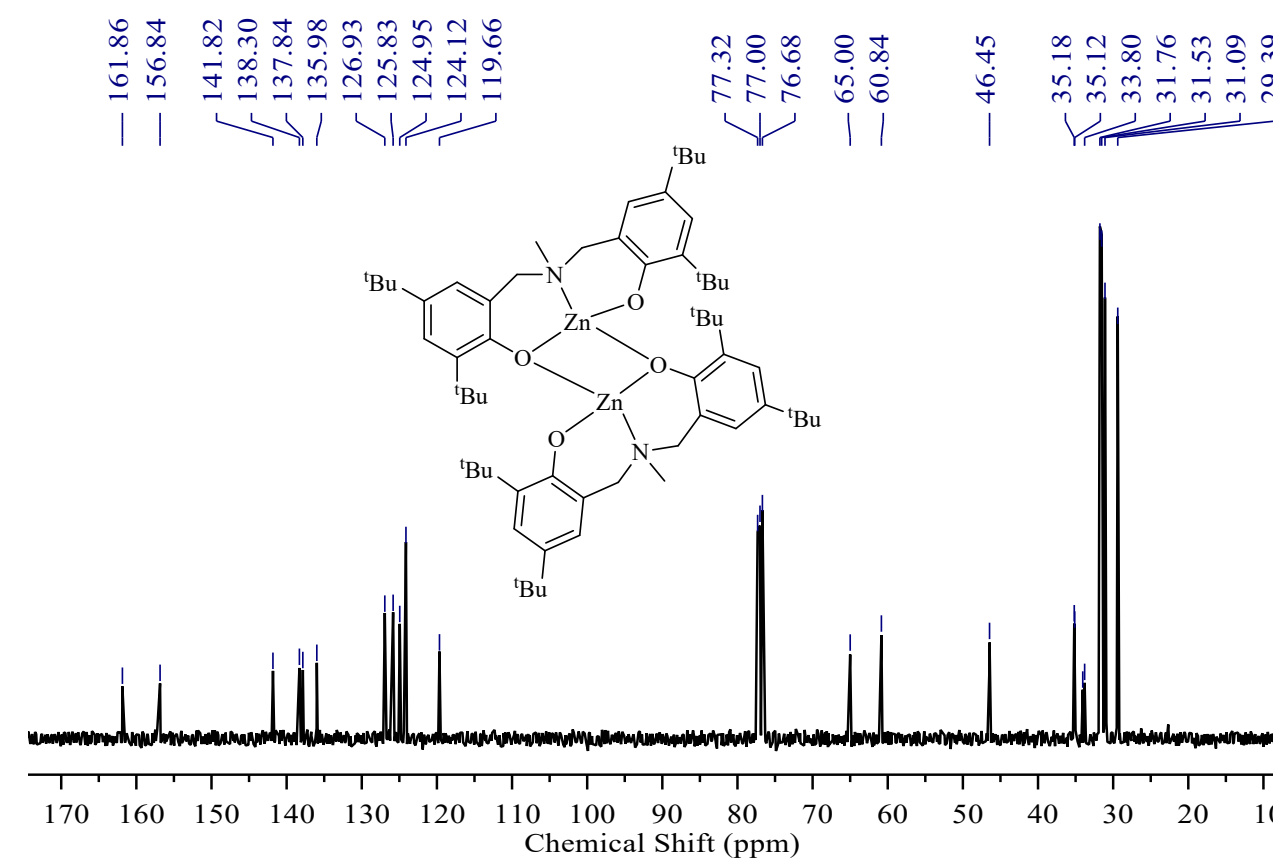

Figure S4. The ${ }^{13} \mathrm{C}$ NMR spectrum of complex $\mathbf{1}\left(\left(\mathbf{L}^{\mathbf{1}}\right)_{\mathbf{2}} \mathbf{Z n}_{\mathbf{2}}\right)\left(100 \mathrm{MHz}, \mathrm{CDCl}_{3}\right)$. 

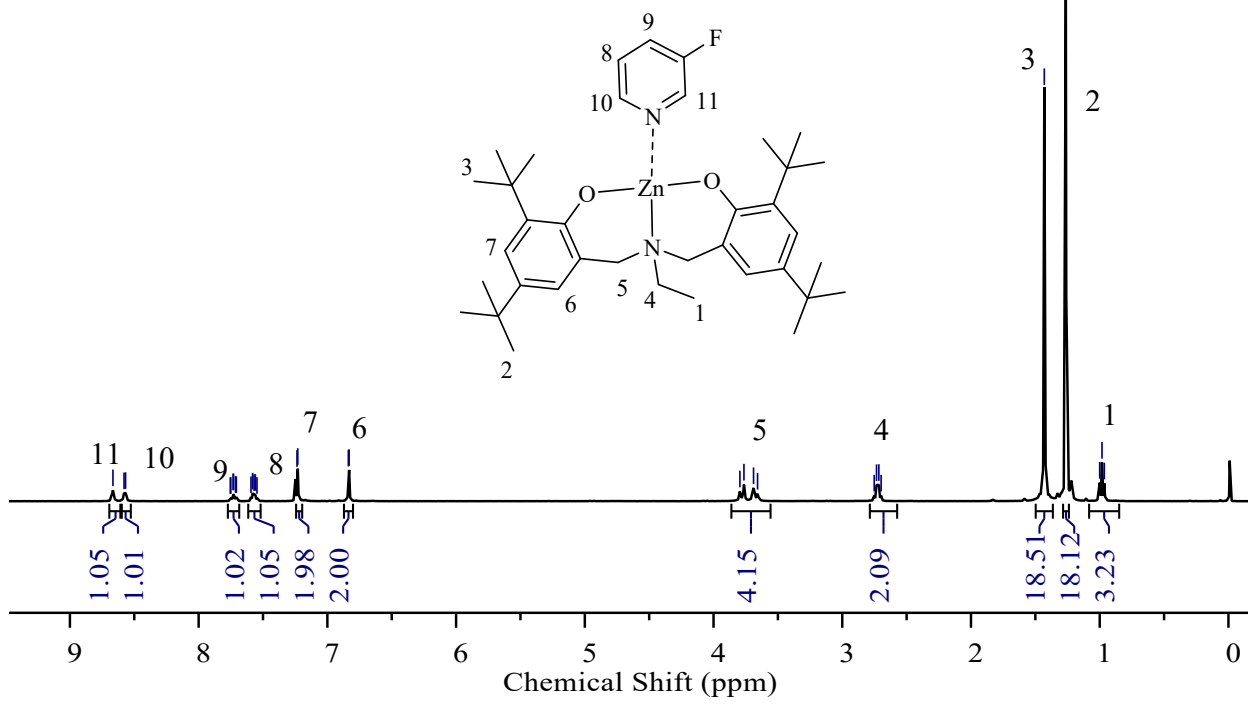

Figure S5. The ${ }^{1} \mathrm{H}$ NMR spectrum of the adduct of $\mathbf{L}^{2} \mathbf{Z n}+3-\mathrm{F}-\mathrm{Py}\left(400 \mathrm{MHz}, \mathrm{CDCl}_{3}\right)$.
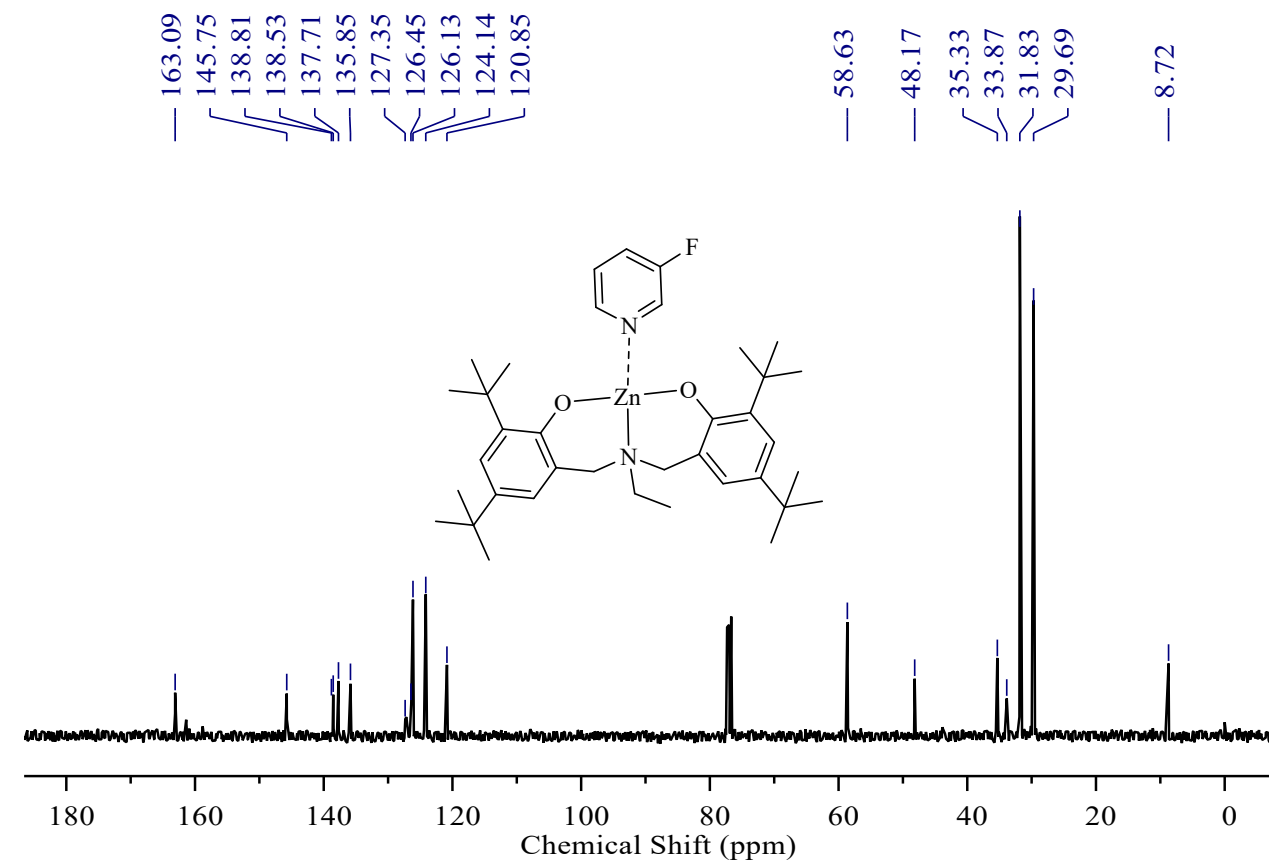

Figure S6. The ${ }^{13} \mathrm{C}$ NMR spectrum of the adduct of $\mathbf{L}^{2} \mathbf{Z n}+3$-F-Py $\left(100 \mathrm{MHz}, \mathrm{CDCl}_{3}\right)$. 


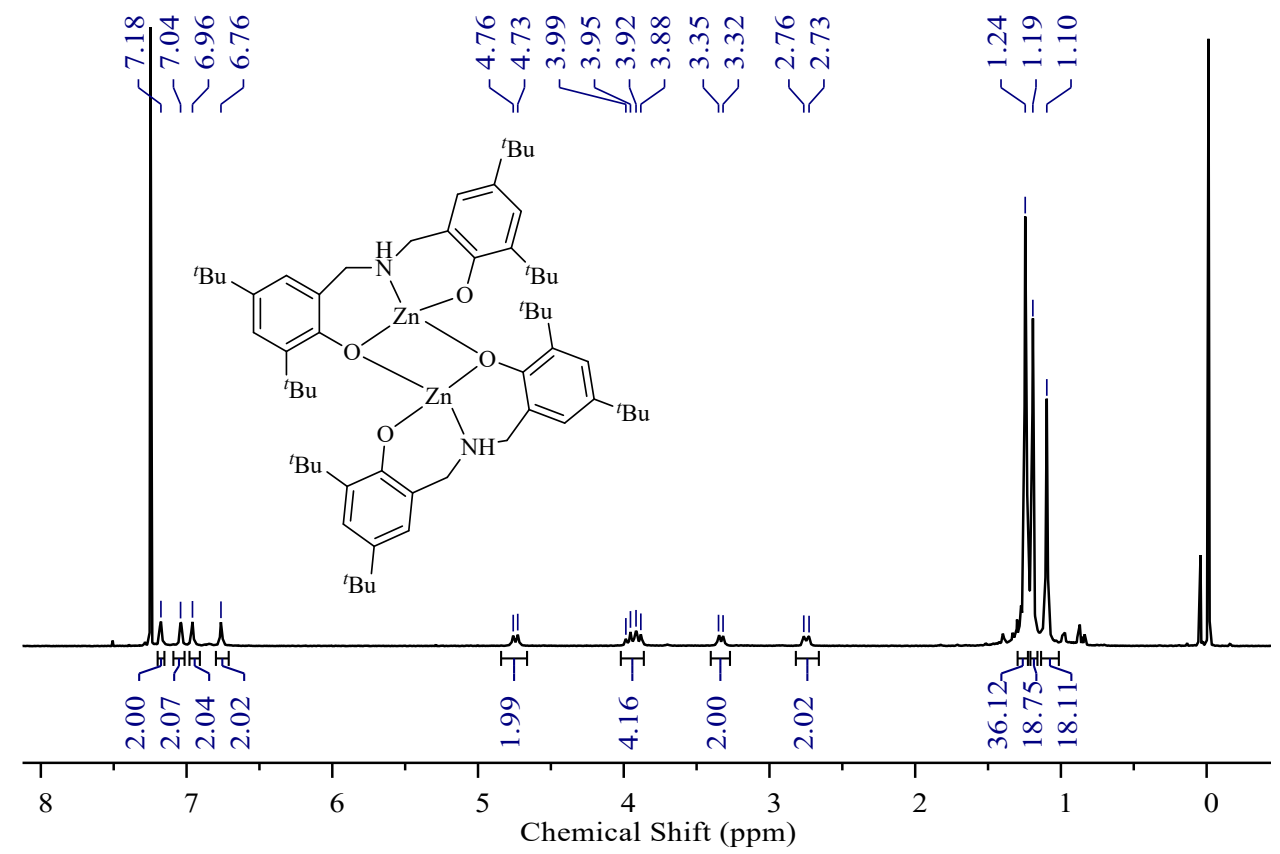

Figure S7. The ${ }^{1} \mathrm{H}$ NMR spectrum of complex $3\left(\left(\mathbf{L}^{3}\right)_{2} \mathbf{Z n}_{2}\right)\left(400 \mathrm{MHz}, \mathrm{CDCl}_{3}\right)$.

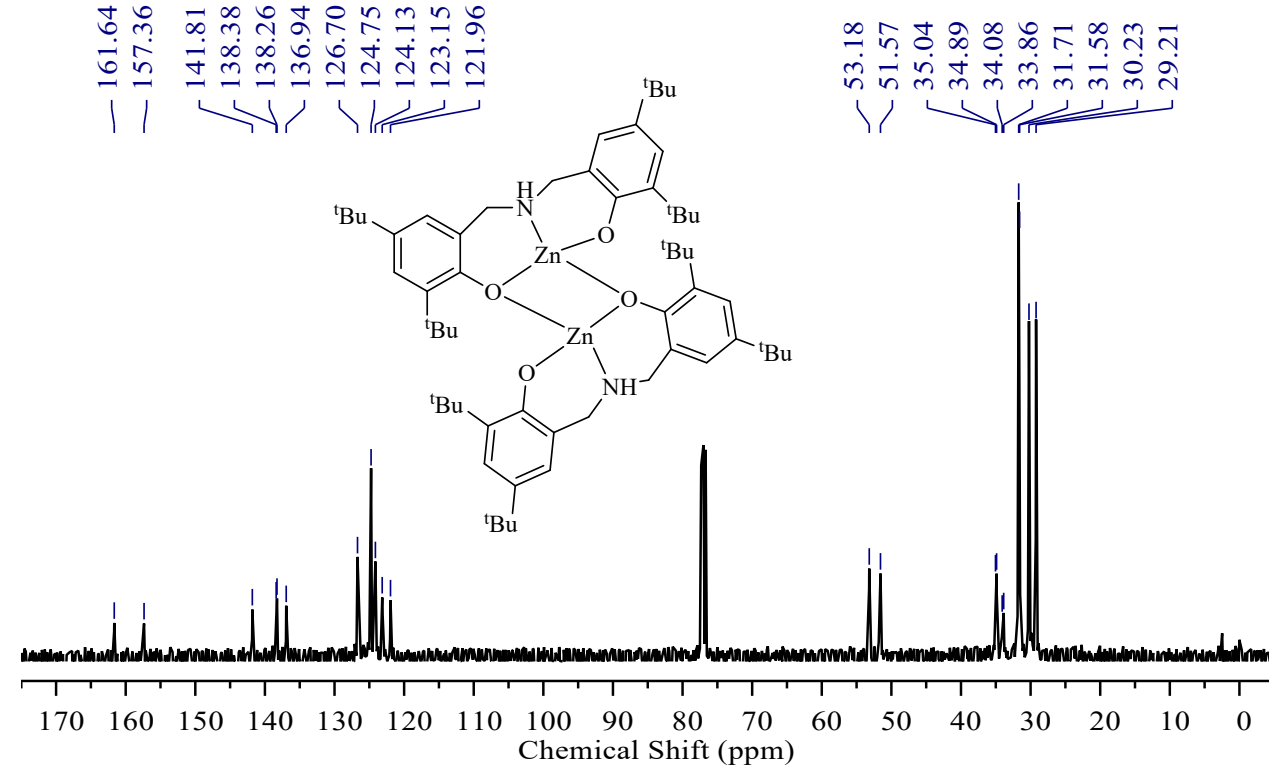

Figure S8. The ${ }^{13} \mathrm{C}$ NMR spectrum of complex $3\left(\left(\mathbf{L}^{\mathbf{3}}\right)_{2} \mathbf{Z n}_{2}\right)\left(100 \mathrm{MHz}, \mathrm{CDCl}_{3}\right)$. 


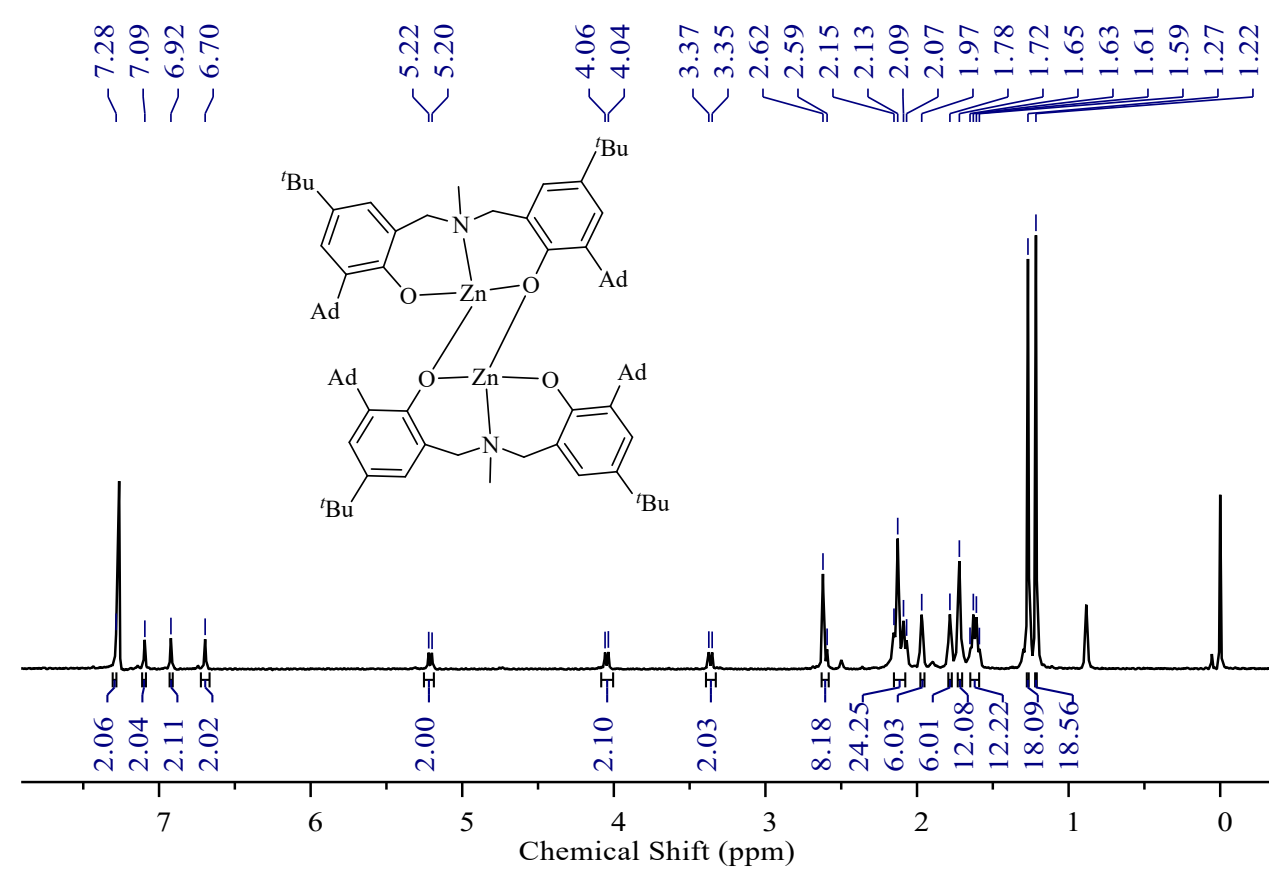

Figure S9. The ${ }^{1} \mathrm{H}$ NMR spectrum of complex $4\left(\left(\mathbf{L}^{4}\right)_{2} \mathbf{Z n}_{2}\right)\left(400 \mathrm{MHz}, \mathrm{CDCl}_{3}\right)$.

ทิ่

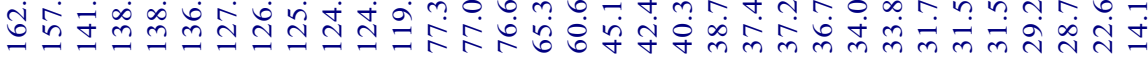

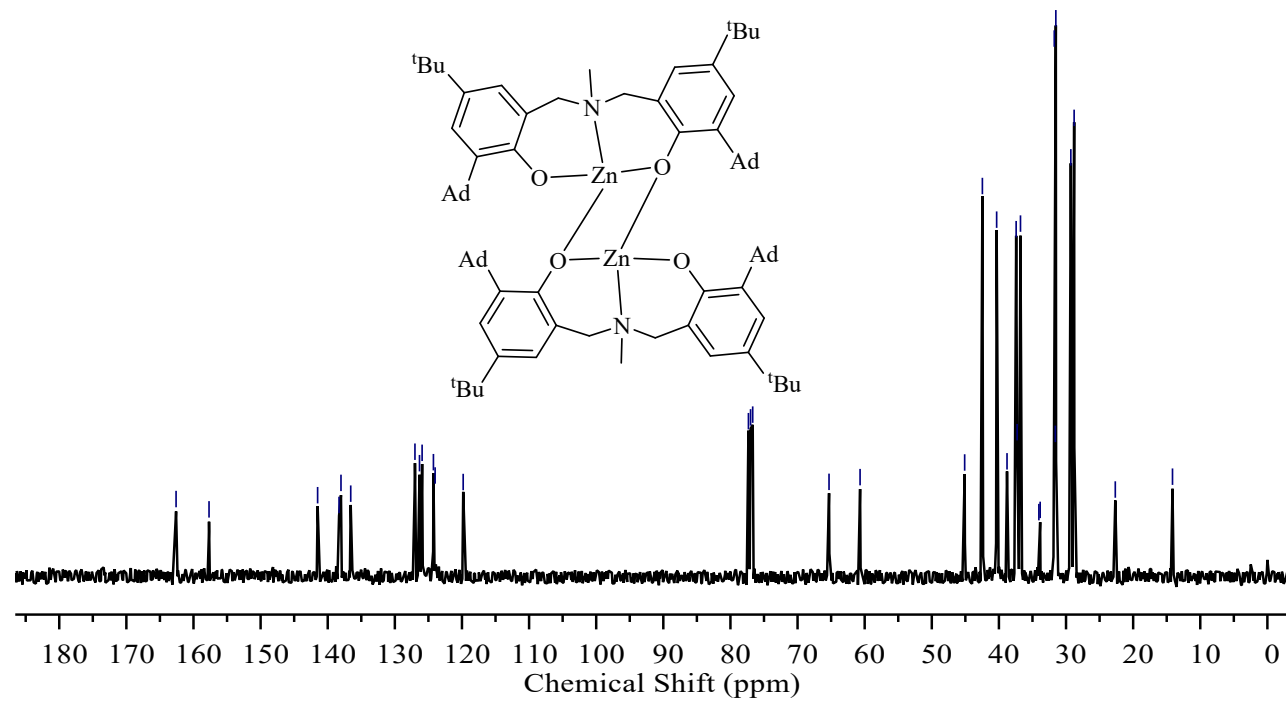

Figure S10. The ${ }^{13} \mathrm{C}$ NMR spectrum of complex $4\left(\left(\mathbf{L}^{4}\right)_{2} \mathbf{Z n}_{2}\right)\left(100 \mathrm{MHz}, \mathrm{CDCl}_{3}\right)$. 


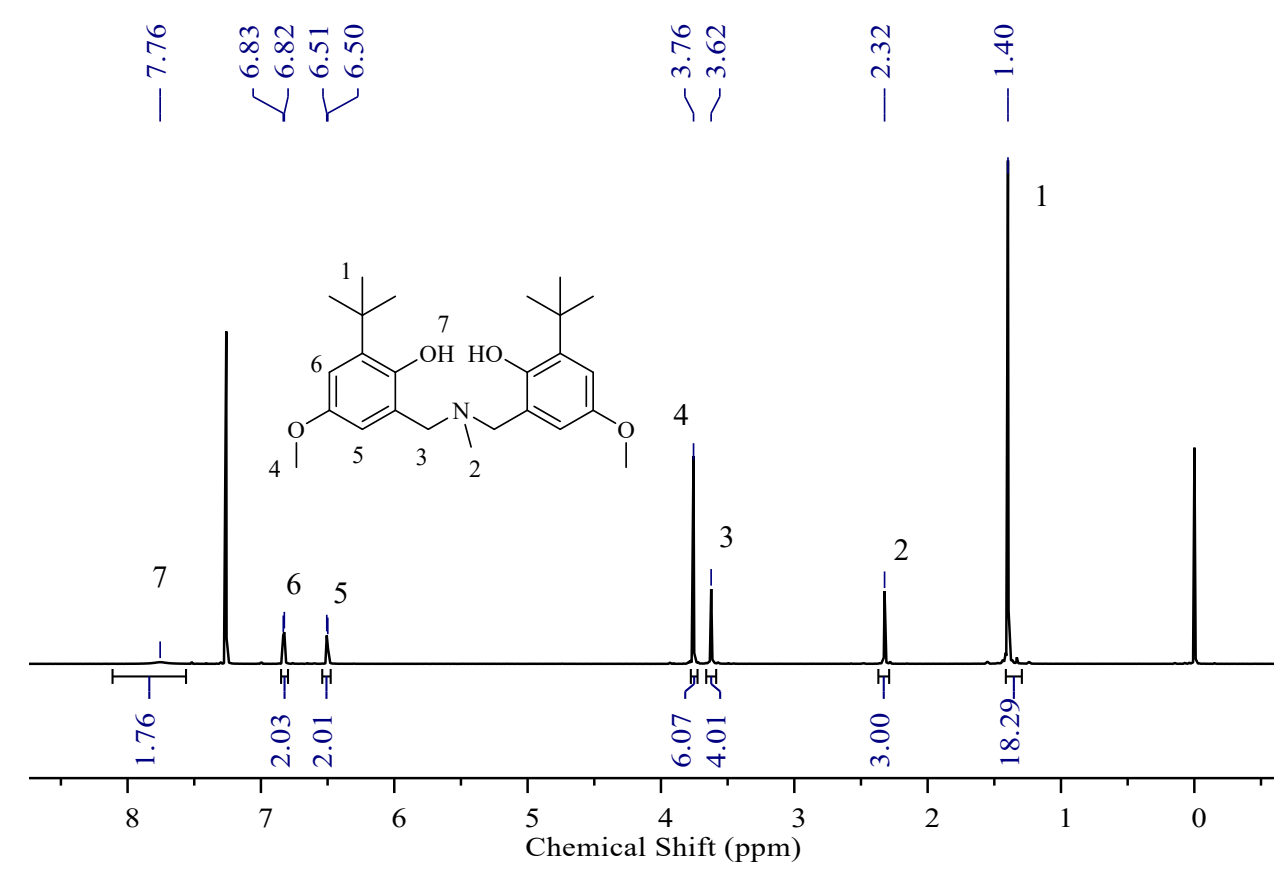

Figure $\mathrm{S} 11$. The ${ }^{1} \mathrm{H}$ NMR spectrum of $\mathrm{H}^{2} \mathrm{~L}_{5}\left(400 \mathrm{MHz}, \mathrm{CDCl}_{3}\right)$.
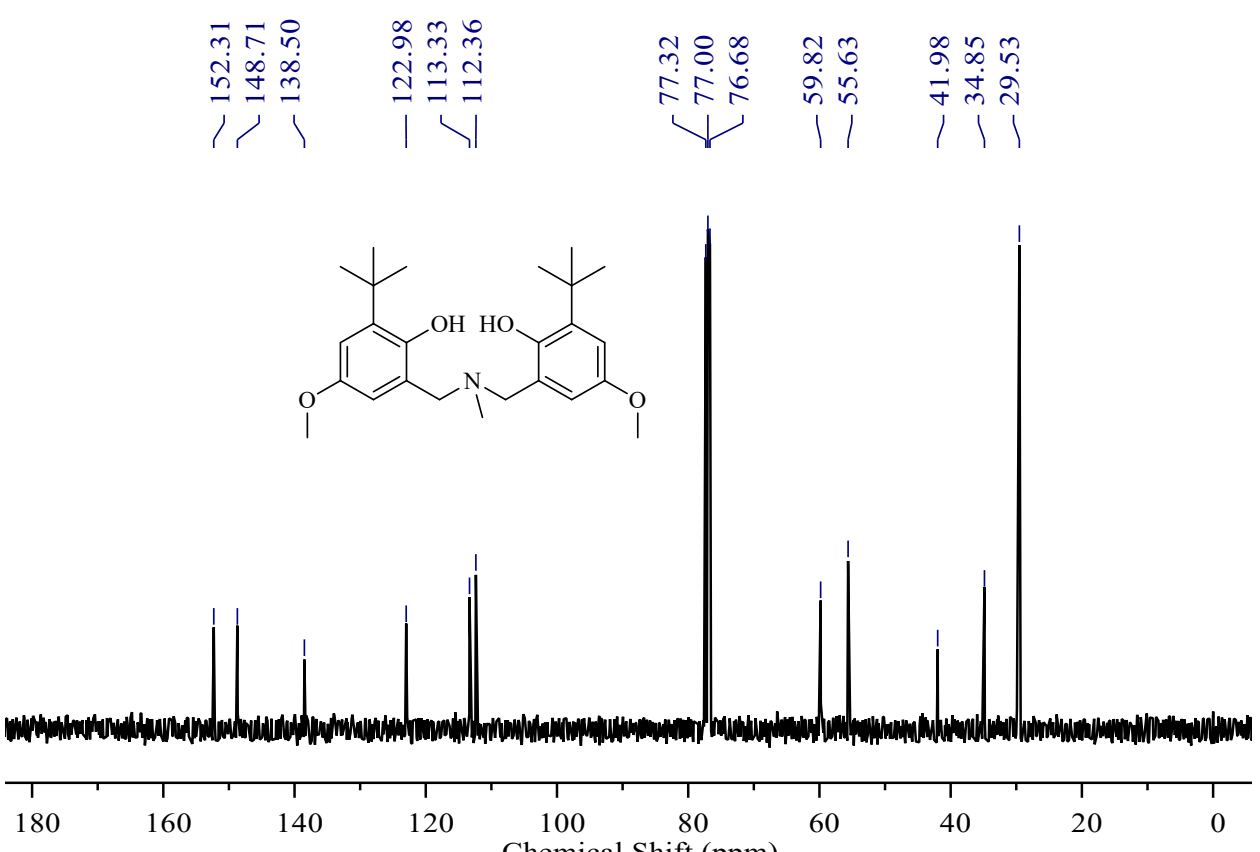

Figure $\mathrm{S} 12$. The ${ }^{13} \mathrm{C} \mathrm{NMR}$ spectrum of $\mathrm{H}^{2} \mathrm{~L}_{5}\left(100 \mathrm{MHz}, \mathrm{CDCl}_{3}\right)$. 


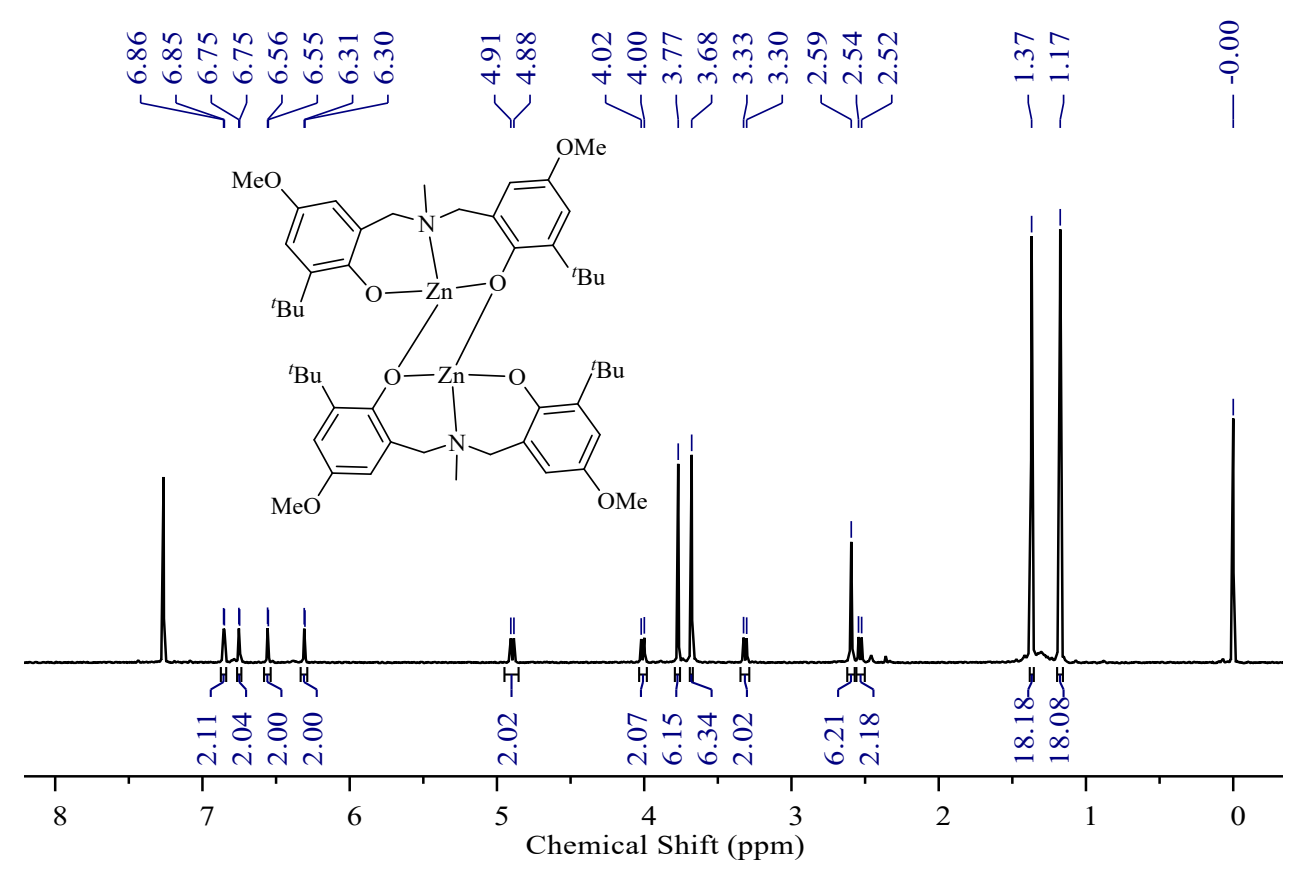

Figure S13. The ${ }^{1} \mathrm{H}$ NMR spectrum of complex $\mathbf{5}\left(\left(\mathbf{L}^{5}\right)_{2} \mathbf{Z n}_{2}\right)\left(400 \mathrm{MHz}, \mathrm{CDCl}_{3}\right)$.

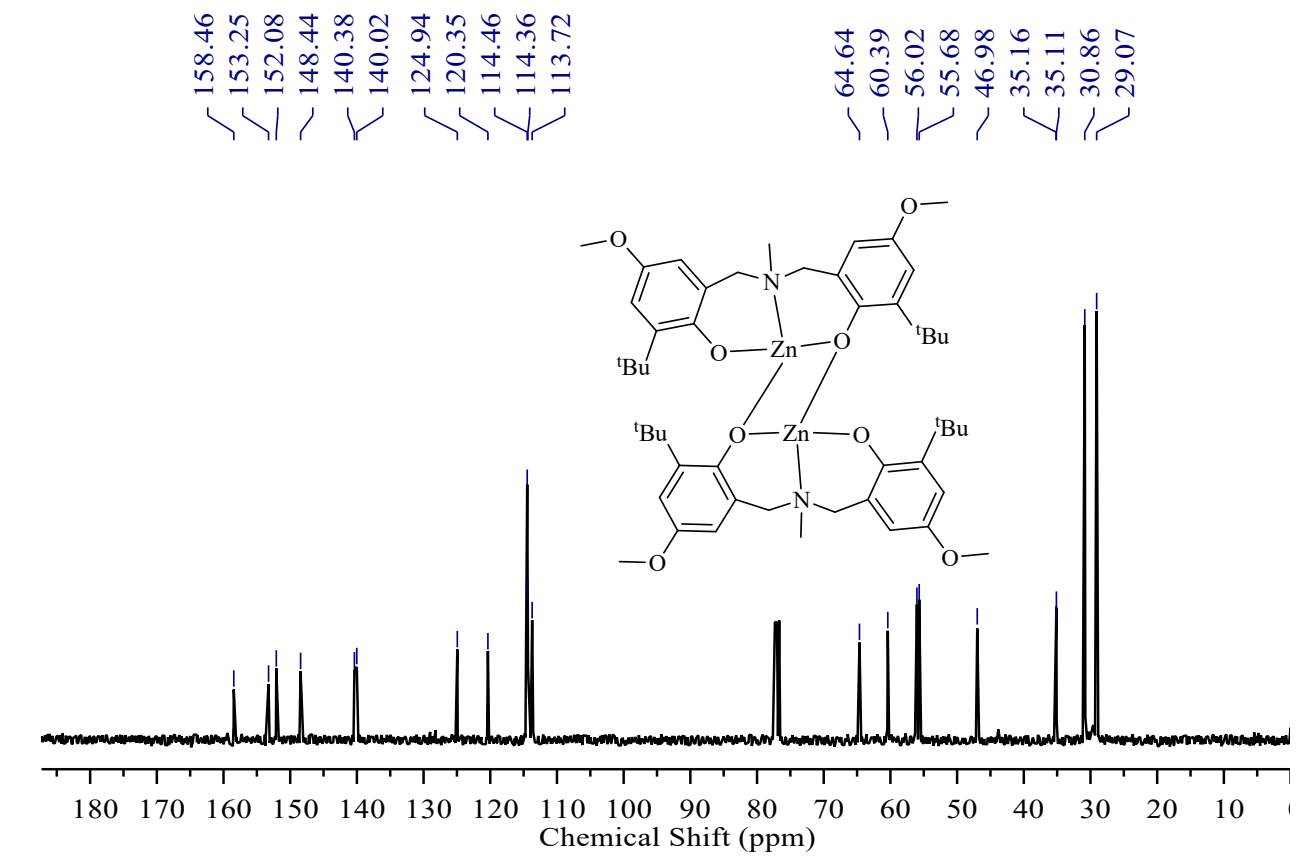

Figure S14. The ${ }^{13} \mathrm{C}$ NMR spectrum of complex $\mathbf{5}\left(\left(\mathbf{L}^{5}\right)_{2} \mathbf{Z n}_{2}\right)\left(100 \mathrm{MHz}, \mathrm{CDCl}_{3}\right)$. 


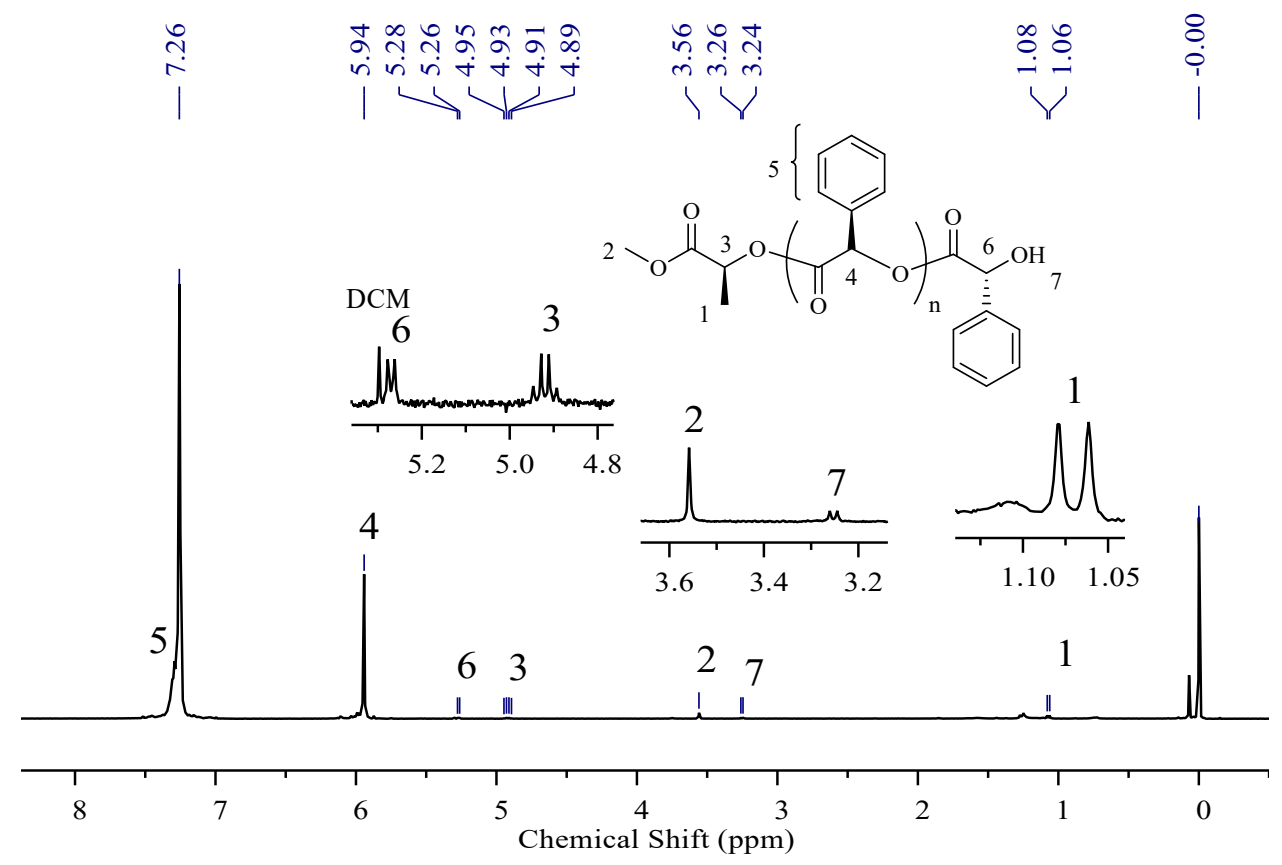

Figure S15. ${ }^{1} \mathrm{H}$ NMR spectrum of poly $\left(\mathrm{D}^{-m a n O C A}\right)$ produced by complex $1\left(400 \mathrm{M}, \mathrm{CDCl}_{3}\right)$.

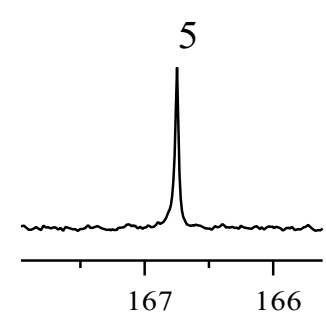

तิ

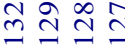

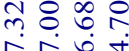

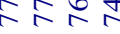

$\lambda \backslash 1$

Nit

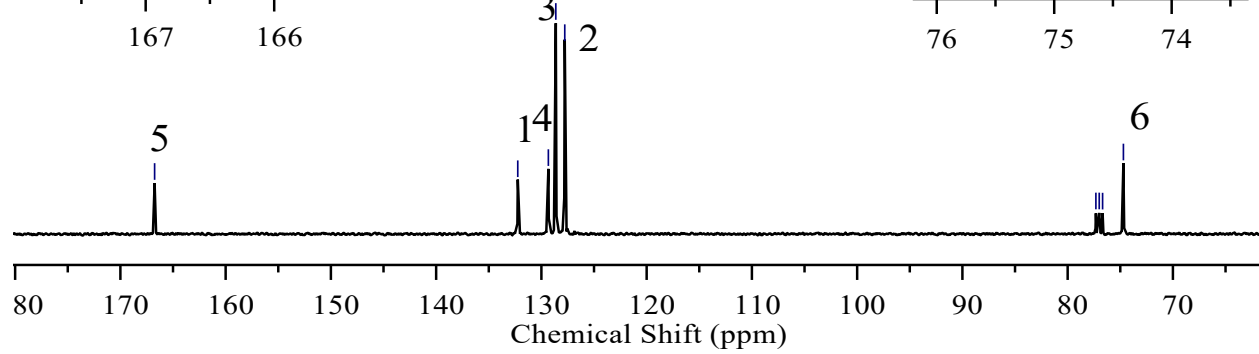

Figure S16. ${ }^{13} \mathrm{C}$ NMR spectrum of poly $\left({ }^{-}\right.$-manOCA) produced by complex $1\left(100 \mathrm{M}, \mathrm{CDCl}_{3}\right)$. 


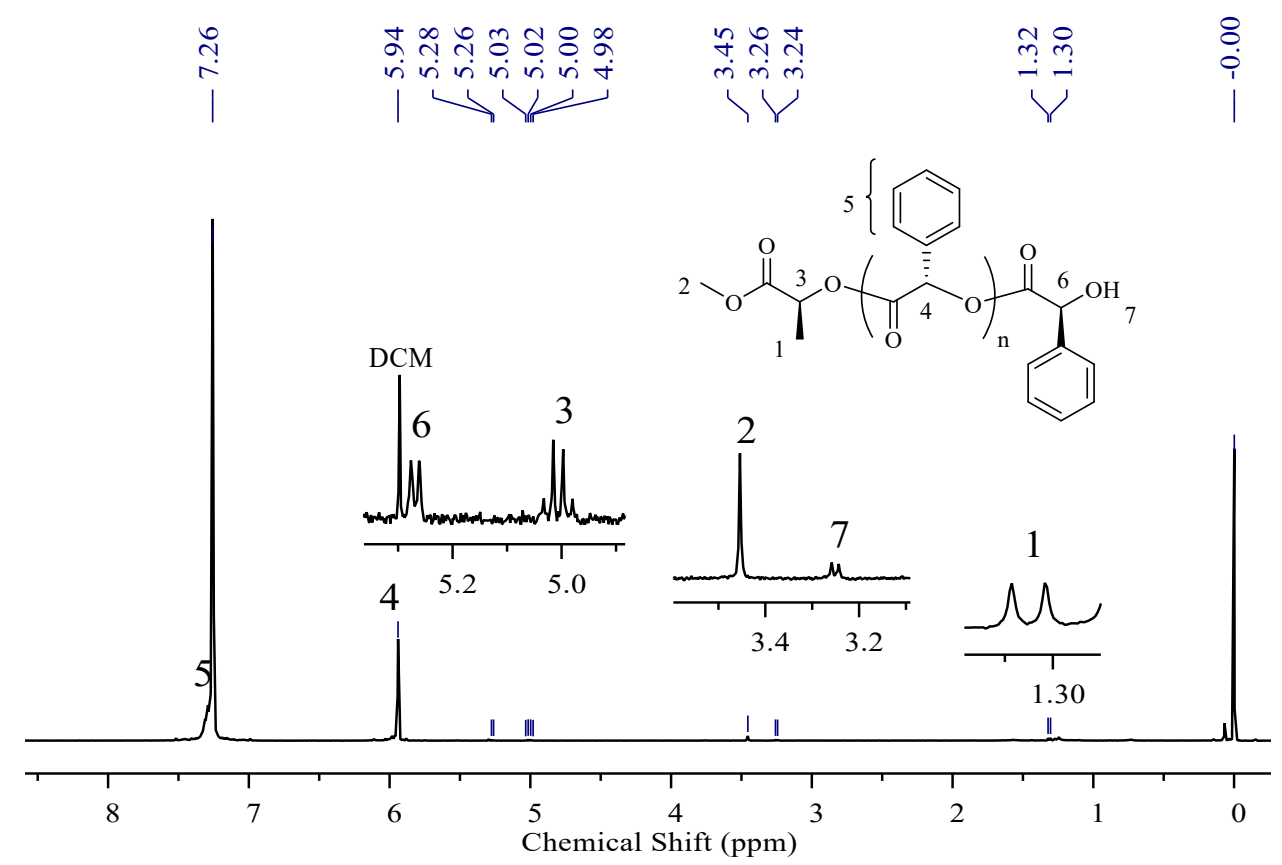

Figure S17. ${ }^{1} \mathrm{H}$ NMR spectrum of poly $\left({ }_{L}-\operatorname{manOCA}\right)$ produced by complex $1\left(400 \mathrm{M}, \mathrm{CDCl}_{3}\right)$.

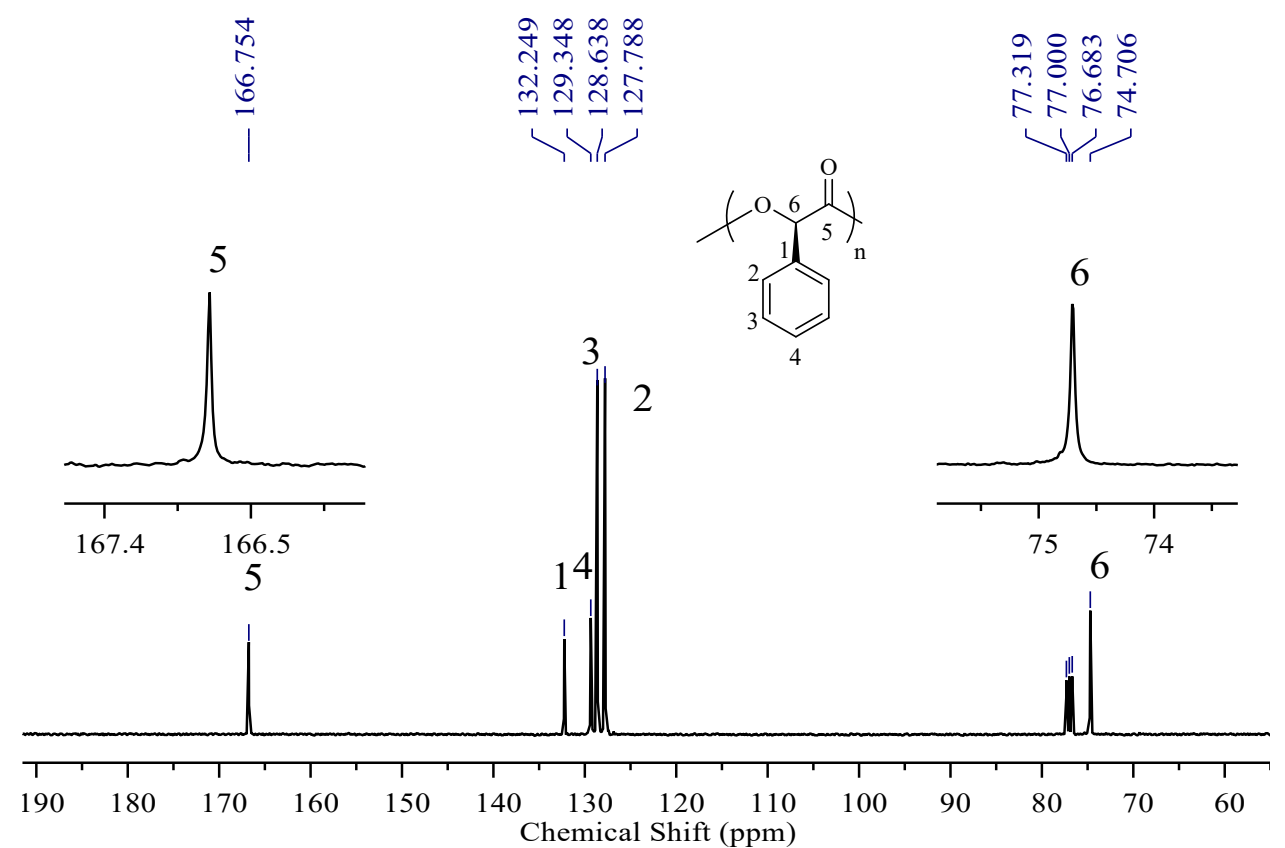

Figure $\mathrm{S} 18 .{ }^{13} \mathrm{C}$ NMR spectrum of poly $(\mathrm{L}-\operatorname{man} \mathrm{OCA})$ produced by complex $1\left(100 \mathrm{M}, \mathrm{CDCl}_{3}\right)$. 


\section{Mechanism studies:}

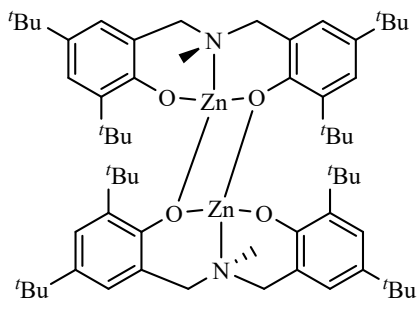

$\left(\mathbf{L}^{1}\right)_{2} \mathrm{Zn}_{2}$
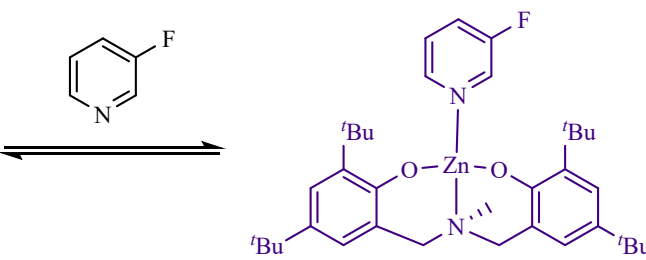

$\mathbf{L}^{1} \mathbf{Z n}+3-\mathrm{F}-\mathrm{Py}$

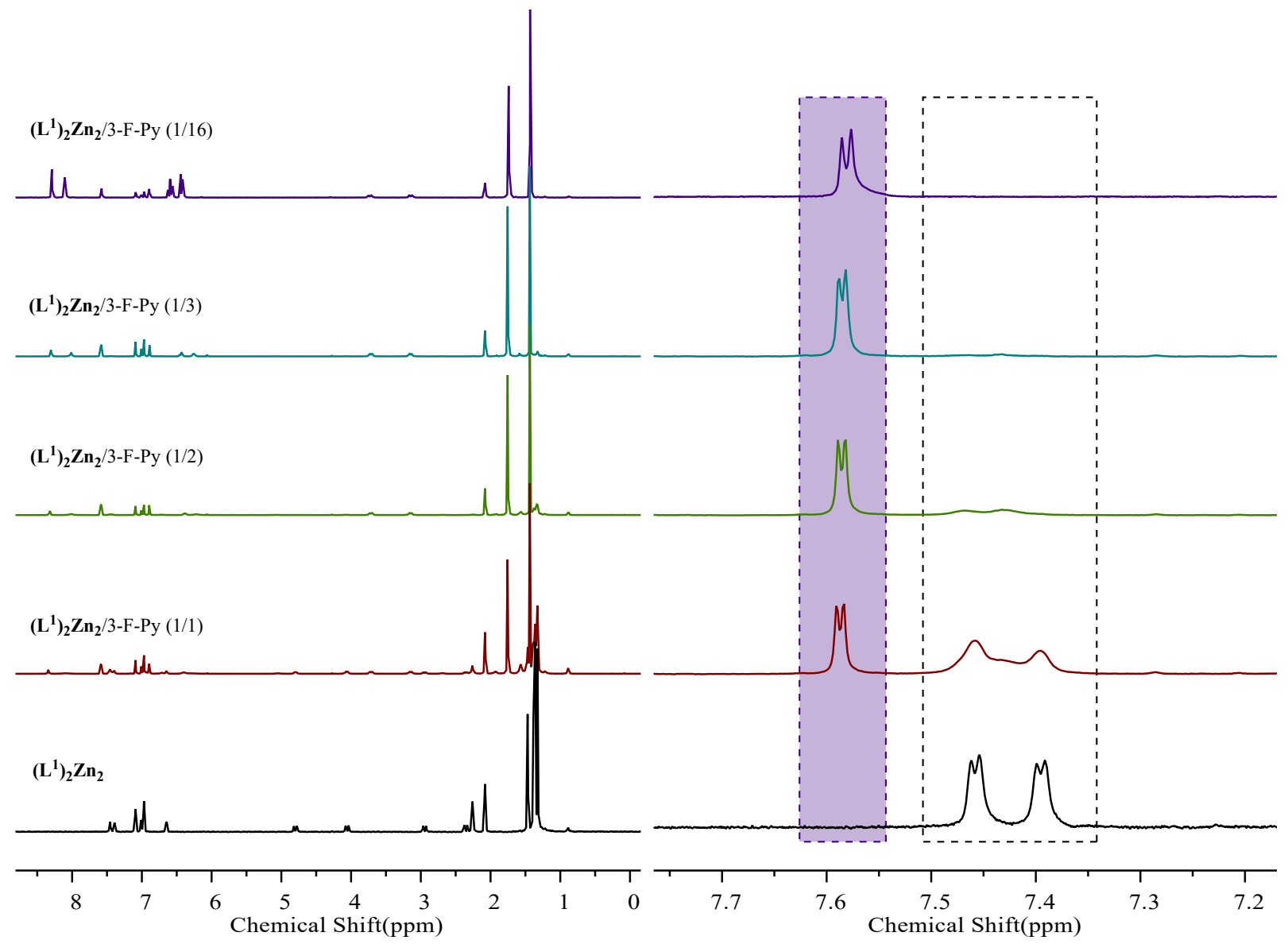

Figure S19. ${ }^{1} \mathrm{H}$ NMR spectra of the adducts of $\left(\mathbf{L}^{1}\right)_{2} \mathbf{Z n}_{\mathbf{2}}$ and 3-fluoropyridine with different ratios in toluene- $d_{8}$. 


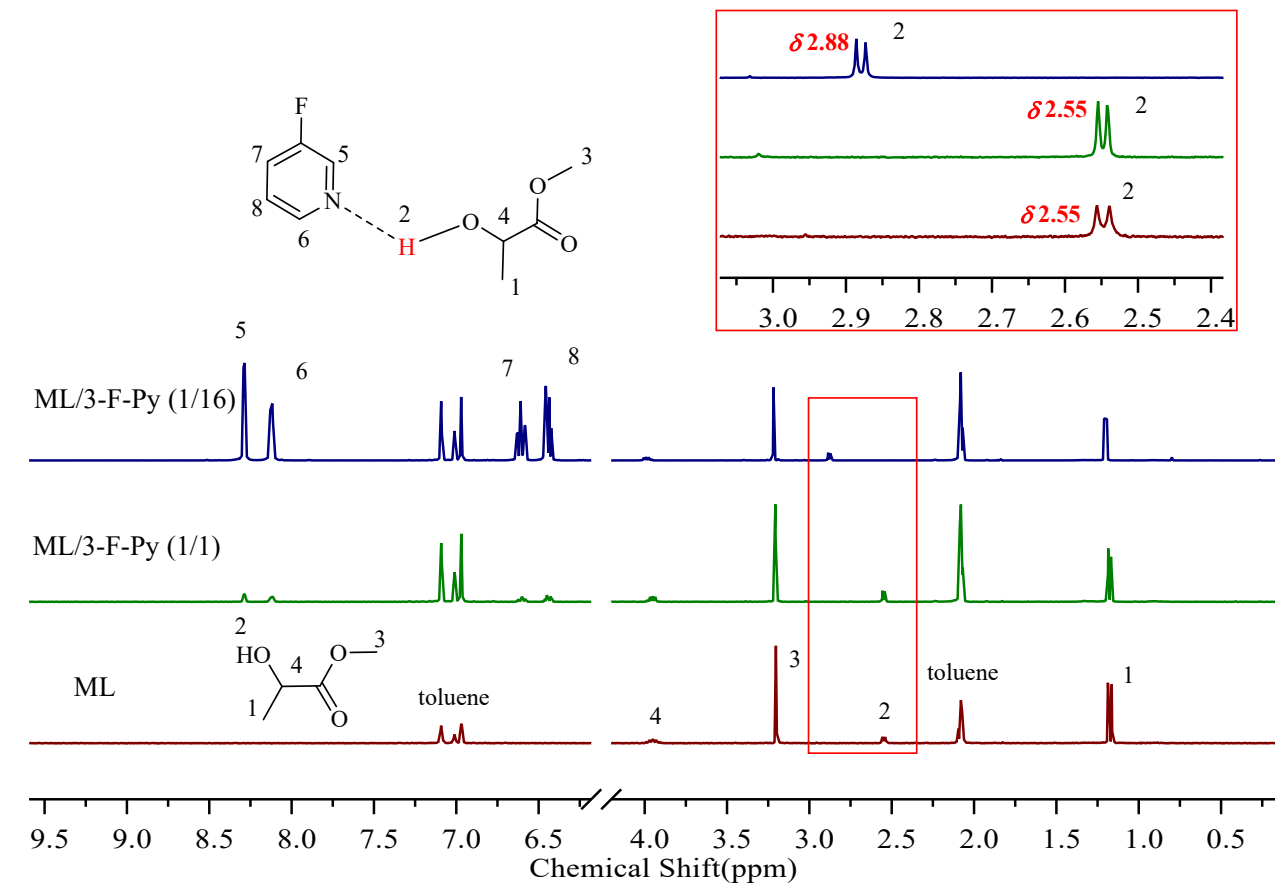

Figure S20. ${ }^{1} \mathrm{H}$ NMR spectra of the mixture of 3-F-Py and $\mathrm{L}_{\mathrm{H}} \mathrm{ML}$ with different ratios in toluene- $d_{8}$.

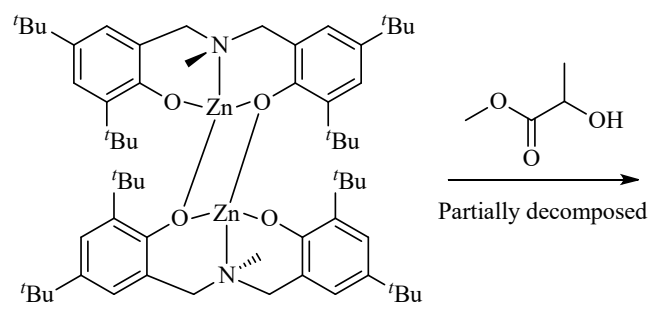

$\left(\mathbf{L}^{1}\right)_{2} \mathbf{Z n}_{2}$

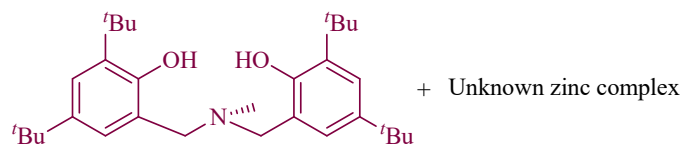

$\mathrm{H}^{2} \mathrm{~L}_{1}$

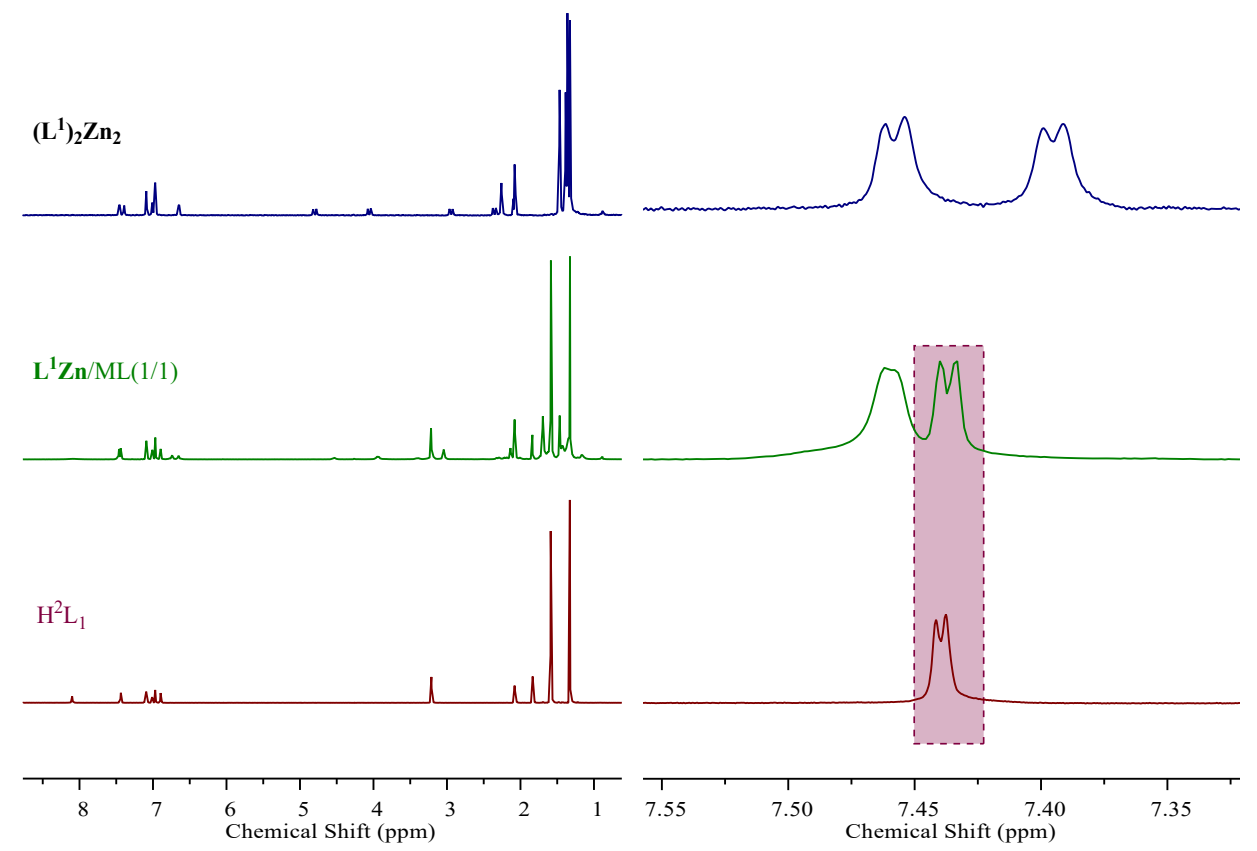

Figure S21. ${ }^{1} \mathrm{H}$ NMR spectra of the mixture of $\left(\mathbf{L}^{\mathbf{1}}\right)_{\mathbf{2}} \mathbf{Z} \mathbf{n}_{\mathbf{2}}$ and ${ }_{\mathrm{L}}-\mathrm{ML}$ which shows $\left(\mathbf{L}^{\mathbf{1}}\right)_{2} \mathbf{Z} \mathbf{n}_{\mathbf{2}}$ was partially decomposed into $\mathrm{H}^{2} \mathrm{~L}_{1}$ upon addition of $\mathrm{ML}$ to $\left(\mathbf{L}^{\mathbf{1}}\right)_{2} \mathbf{Z n}_{\mathbf{2}}$ in toluene- $d_{8}$ solution. 


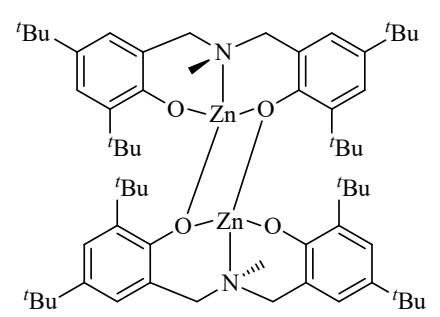

$\left(\mathbf{L}^{1}\right)_{2} \mathbf{Z} \mathbf{n}_{2}$
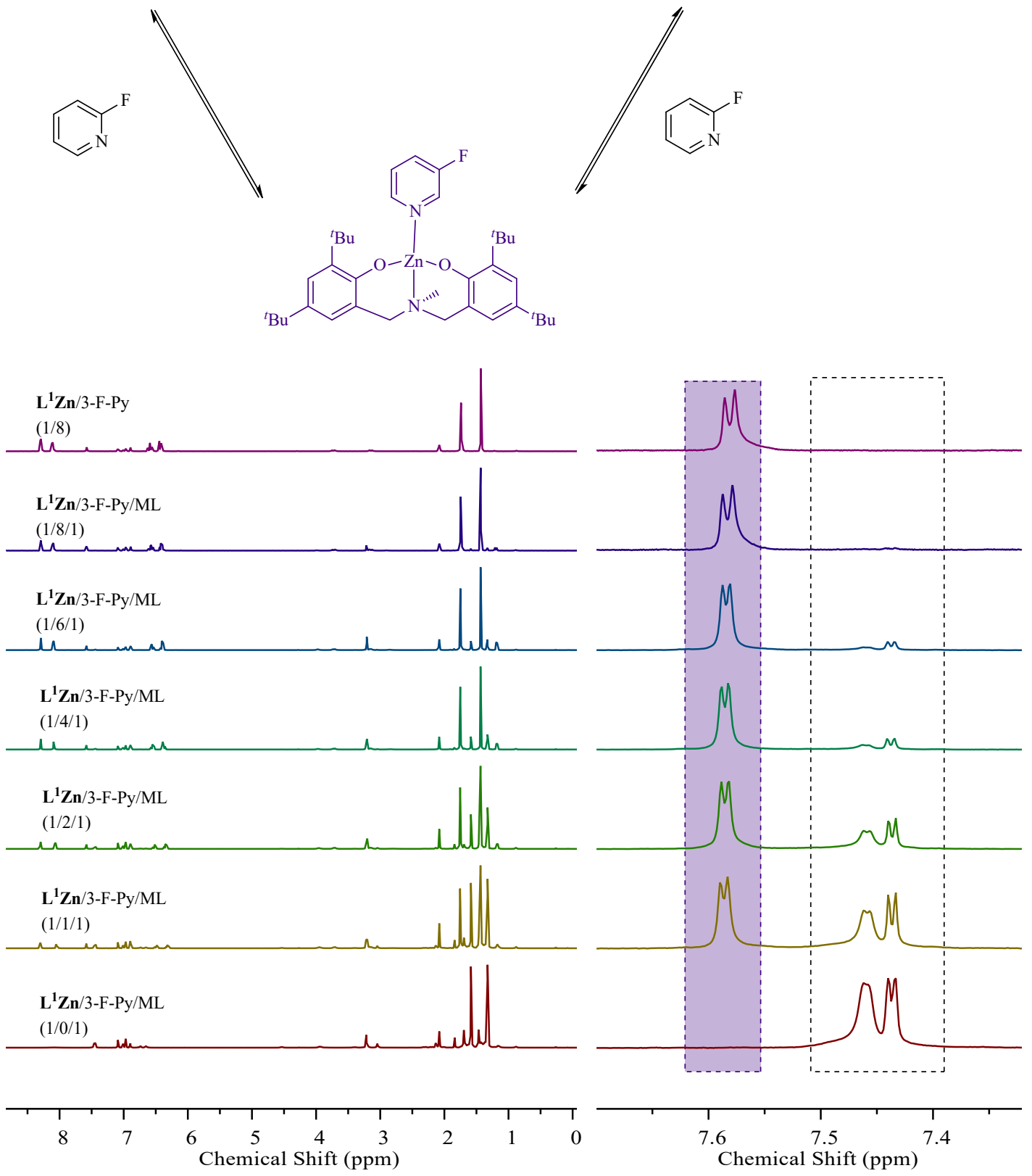

Figure S22. ${ }^{1} \mathrm{H}$ NMR spectra in toluene- $d_{8}$ to prove the reversion of complex $\mathbf{1}$ to the adduct $\mathbf{L}^{\mathbf{1}} \mathbf{Z n}$ + 3-F-py via adding different ratios of 3-F-Py to $\left[\mathbf{L}^{1} \mathbf{Z n}\right] /\left[L_{L}-M L\right](1 / 1)$ mixed solution. 


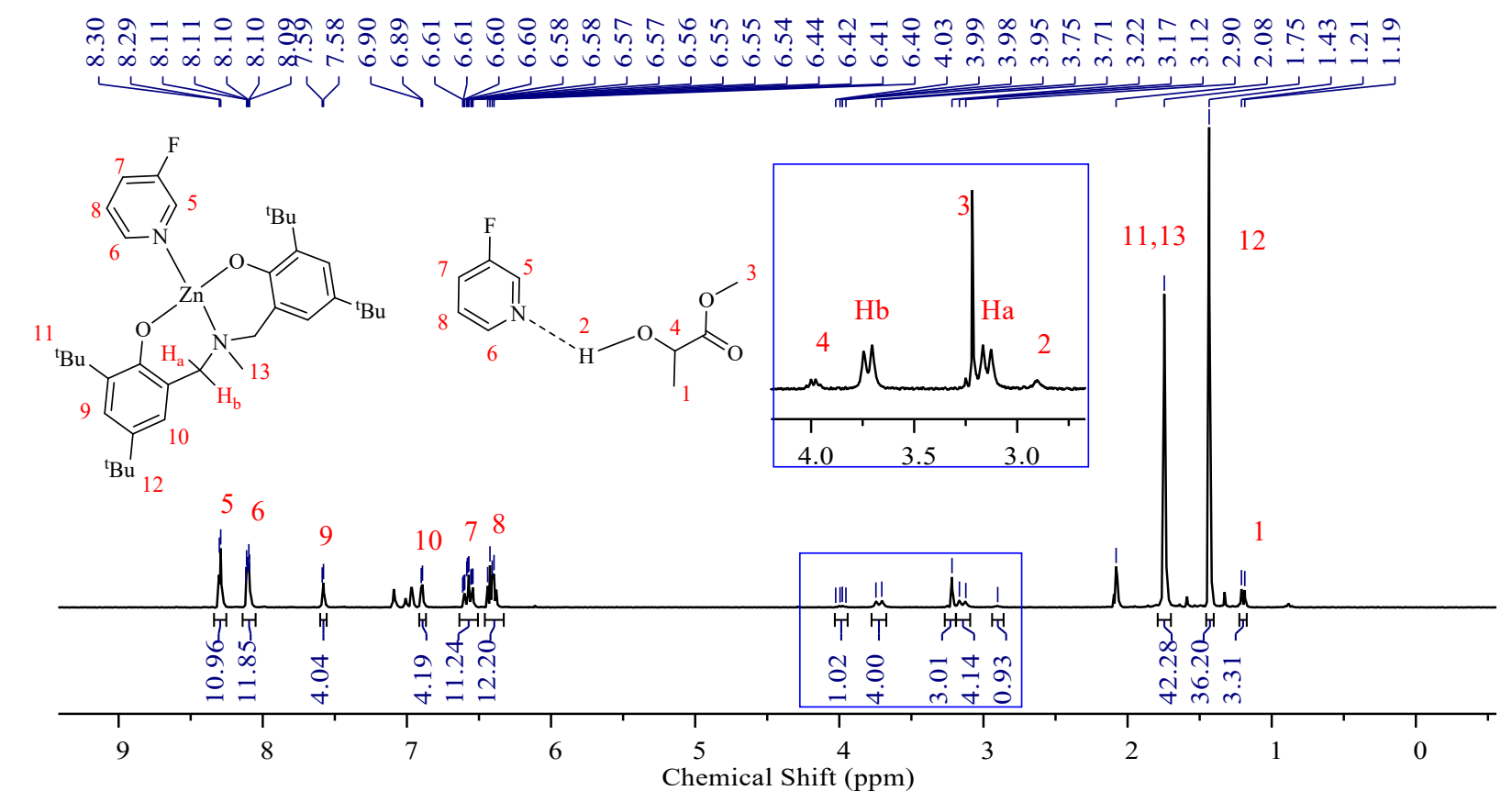

Figure S23. ${ }^{1} \mathrm{H}$ NMR spectrum of the mixture of $\left[\mathbf{L}^{1} \mathbf{Z n}\right] /[3-\mathrm{F}-\mathrm{Py}] /[\mathrm{L}-\mathrm{ML}](1 / 8 / 1)$ in toluene- $d_{8}$. 


\section{Controlled polymerization research}

Table S1. ROP of $\mathrm{L}_{-}$manOCA (entries 1-12), $\mathrm{D}^{-m a n O C A}$ (entry 14), and rac-manOCA (entry 13) by $\mathbf{1}{ }^{a}$

\begin{tabular}{|c|c|c|c|c|c|c|c|c|c|}
\hline Entry & $\begin{array}{l}{[\mathrm{Zn}]_{0} /[3-\mathrm{F}-\mathrm{Py}]_{0} /[\mathrm{L}-\mathrm{ML}]_{0} /[} \\
\mathrm{OCA}]_{0}\end{array}$ & Solvent & Time (h) & $\begin{array}{l}\text { Conv. }{ }^{b} \\
(\%)\end{array}$ & $\begin{array}{l}M_{\mathrm{n}, \mathrm{obsd}}{ }^{c} \\
(\mathrm{~g} / \mathrm{mol})\end{array}$ & $\begin{array}{l}M_{\mathrm{n}, \mathrm{NMR}}{ }^{d} \\
(\mathrm{~g} / \mathrm{mol})\end{array}$ & $\begin{array}{l}M_{\mathrm{n}, \text { calcd }^{e}} \\
(\mathrm{~g} / \mathrm{mol})\end{array}$ & $Ð$ & $P_{\mathrm{m}}^{f}$ \\
\hline 1 & 1:0:0:50 & TOL & 0.5 & 7 & - & - & - & - & - \\
\hline 2 & $1: 1: 0: 50$ & TOL & 0.5 & 26 & 7300 & - & - & 1.23 & - \\
\hline 3 & $1: 0: 1: 50$ & TOL & 0.5 & 19 & - & - & - & - & - \\
\hline 4 & $0: 1: 1: 50$ & TOL & 0.5 & 0 & - & - & - & - & - \\
\hline 5 & $1: 1: 1: 50$ & DCM & 0.5 & 88 & 5900 & 6700 & 6000 & 1.17 & 0.85 \\
\hline 6 & $1: 1: 1: 50$ & THF & 0.5 & 0 & - & - & - & - & - \\
\hline 7 & $1: 1: 1: 25$ & TOL & $15 \mathrm{~min}$ & 99 & 3100 & 3200 & 3400 & 1.06 & 0.99 \\
\hline $8^{g}$ & 1(1):1:1:100 & TOL & 2.0 & 99 & 14000 & 14300 & 13400 & 1.16 & 0.99 \\
\hline $9^{h}$ & 1(1):1:1:200 & TOL & 5.0 & 99 & 24200 & 28000 & 26600 & 1.13 & 0.99 \\
\hline $10^{g}$ & 1(1):1:1:150 & TOL & 3.0 & 99 & 21000 & 22000 & 19900 & 1.10 & 0.99 \\
\hline 11 & $1: 8: 1: 50$ & TOL & $20 \mathrm{~min}$ & 99 & 7000 & 7200 & 6700 & 1.09 & 0.99 \\
\hline $12^{i}$ & $1: 8: 1: 50$ & TOL & $20 \mathrm{~min}$ & 80 & 5200 & 5000 & 5500 & 1.06 & 0.95 \\
\hline $\begin{array}{l}13(\text { rac-manOC } \\
\text { A })^{j}\end{array}$ & $1: 8: 1: 50$ & TOL & 2.0 & 85 & 5400 & 5300 & 5700 & 1.06 & 0.70 \\
\hline $14(\mathrm{D}-\mathrm{manOCA})$ & $1: 1: 1: 50$ & TOL & 0.5 & 84 & 5400 & 5600 & 5700 & 1.11 & 0.99 \\
\hline 15 & $1: 8: 1: 100$ & TOL & $40 \mathrm{~min}$ & 99 & 11200 & 12000 & 13400 & 1.15 & 0.99 \\
\hline \multicolumn{10}{|c|}{ 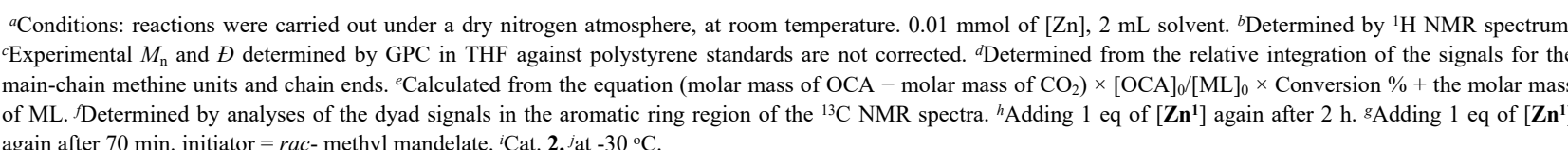 } \\
\hline
\end{tabular}

A)

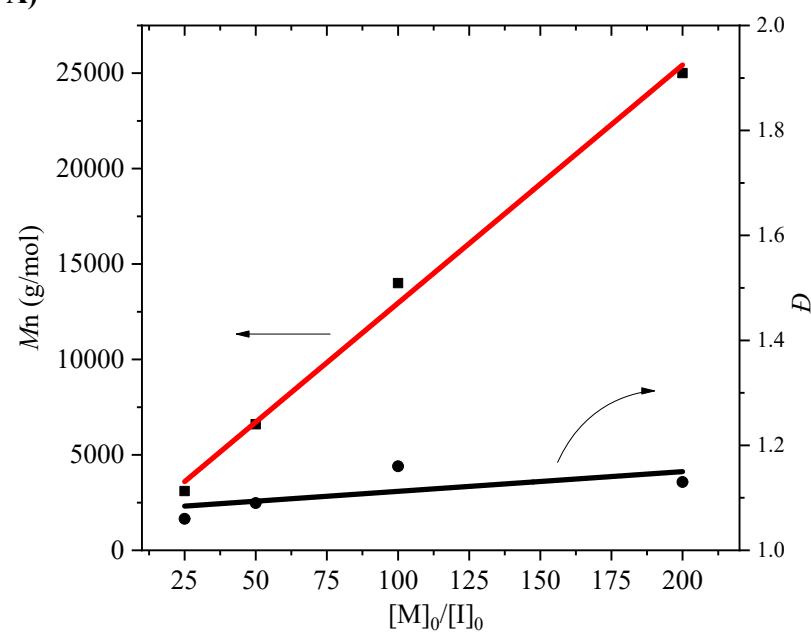

B)

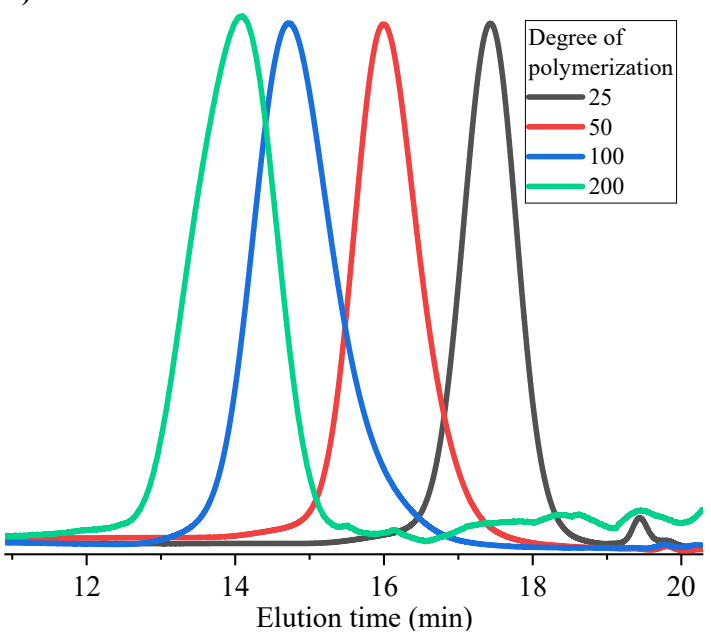

Figure S24. A): Plots of $M_{\mathrm{n}}$ and molecular weight distribution $Ð$ of poly(L-manOCA) versus $[\mathrm{M}]_{0} /[\mathrm{I}]_{0}$ ratio. B): Representative gel permeation chromatography (GPC) traces of the poly $\left(\mathrm{L}_{-}-\right.$man $\left.\mathrm{OCA}\right)$ prepared by complex $\mathbf{1}$. 


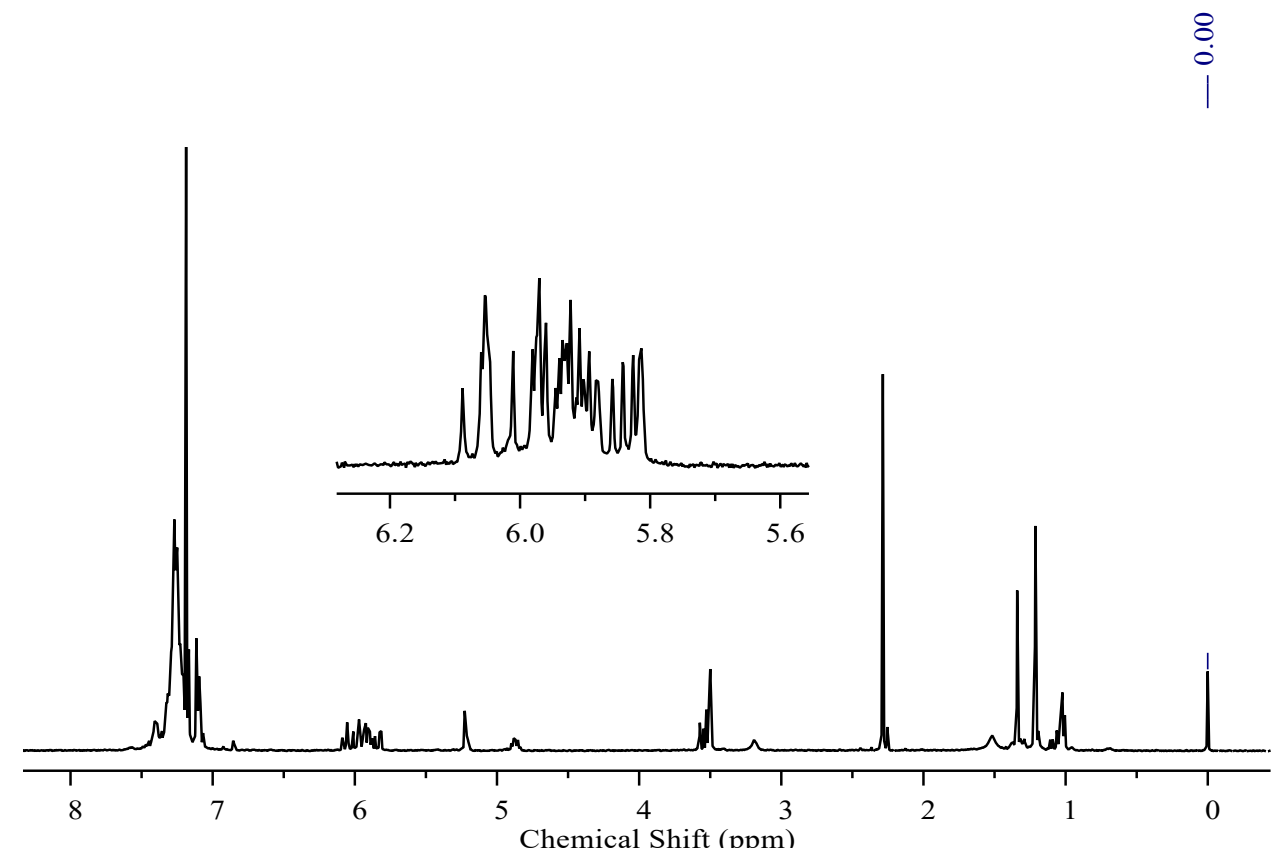

Figure S25. The ${ }^{1} \mathrm{H} \quad \mathrm{NMR}$ spectrum $\left(\mathrm{CDCl}_{3}, 400 \mathrm{M}\right)$ of poly(L-manOCA). $\left(\left[\mathbf{L}^{1} \mathbf{Z n}\right]_{0} /[3-\mathrm{F}-\mathrm{Py}]_{0} /\left[\mathrm{L}_{\mathrm{L}}-\mathrm{ML}\right]_{0} /[\mathrm{OCA}]_{0}=1: 0: 1: 50\right.$, time $\left.=6 \mathrm{~h}\right)$ 


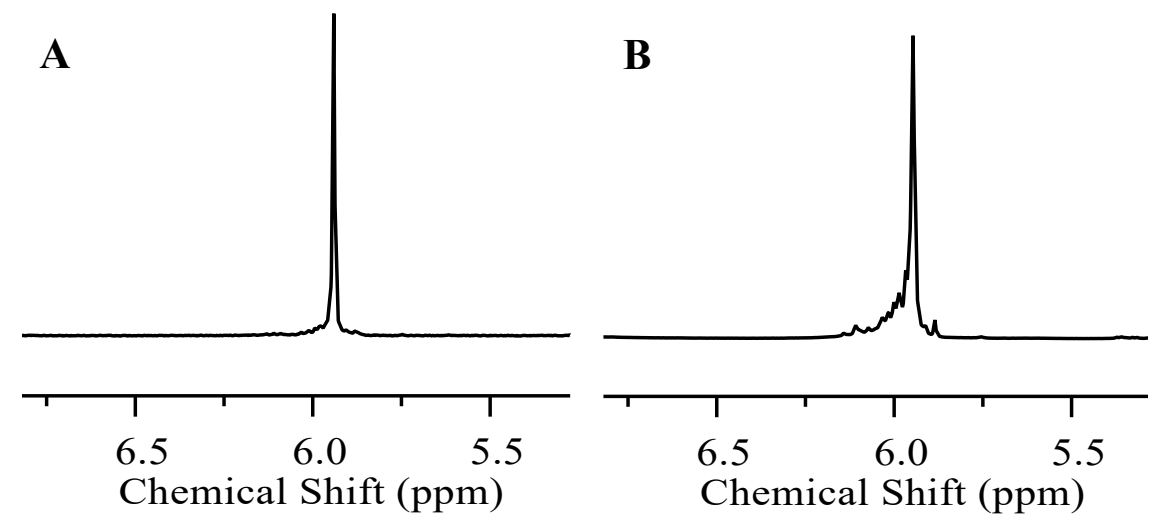

Figure S26. The methine resonances in ${ }^{1} \mathrm{H}$ NMR spectra of poly $(\mathrm{L}-$ man $\mathrm{OCA})$ obtained by ROP of ${ }_{\mathrm{L}}-\mathrm{manOCA}$ at the ratio of $[\mathrm{Zn}]_{0} /[3-\mathrm{F}-\mathrm{Py}]_{0} /[\mathrm{L}-\mathrm{ML}]_{0} /[\mathrm{OCA}]_{0}(1 / 1 / 1 / 50)$ by complex 1 at R.T. in A: TOL (Table 1, entry 1); B: DCM. (Table S1, entry 5)

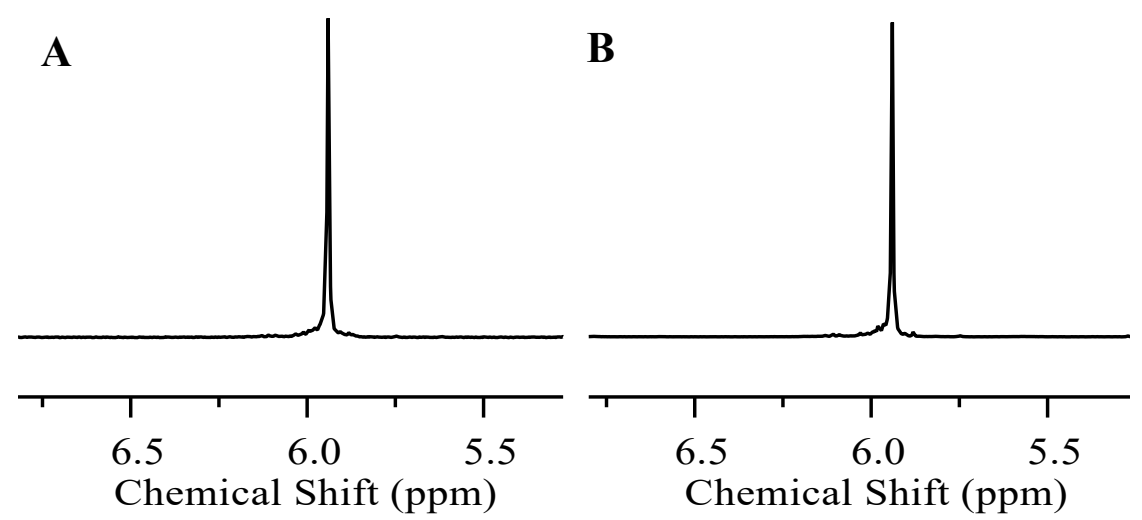

Figure S27. The methine resonances in ${ }^{1} \mathrm{H}$ NMR spectra of poly $(\mathrm{L}-\operatorname{man} \mathrm{OCA})$ obtained by ROP of ${ }_{\mathrm{L}}-\operatorname{manOCA}$ at the ratio of $\mathrm{A}:[\mathrm{Zn}]_{0} /[3-\mathrm{F}-\mathrm{Py}]_{0} /[\mathrm{L}-\mathrm{ML}]_{0} /[\mathrm{OCA}]_{0}(1 / 1 / 1 / 50)$ (Table 1 , entry 1$)$; B: $[\mathrm{Zn}]_{0} /[3-\mathrm{F}-\mathrm{Py}]_{0} /[\mathrm{L}-\mathrm{ML}]_{0} /[\mathrm{OCA}]_{0}(1 / 8 / 1 / 50)$ (Table $\mathrm{S} 1$, entry 11$)$ by complex 1 at R.T.
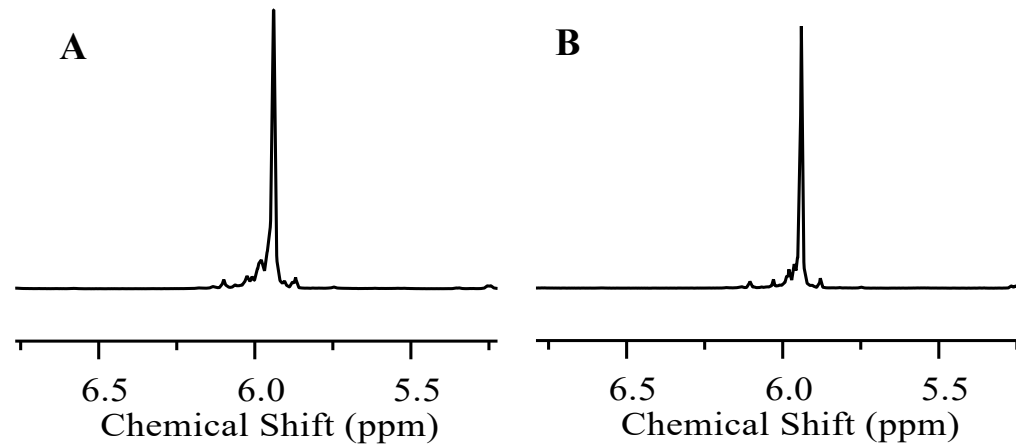

Figure S28. The methine resonances in ${ }^{1} \mathrm{H}$ NMR spectra of poly $(\mathrm{L}-\operatorname{manOCA})$ obtained by ROP of ${ }_{\mathrm{L}}-\operatorname{manOCA}$ at the ratio of $\mathrm{A}:[\mathrm{Zn}]_{0} /[3-\mathrm{F}-\mathrm{Py}]_{0} /[\mathrm{L}-\mathrm{ML}]_{0} /[\mathrm{OCA}]_{0}(1 / 1 / 1 / 50)$ (Table 1 , entry 2$)$; B: $[\mathrm{Zn}]_{0} /[3-\mathrm{F}-\mathrm{Py}]_{0} /[\mathrm{L}-\mathrm{ML}]_{0} /[\mathrm{OCA}]_{0}(1 / 8 / 1 / 50)$ (Table $\mathrm{S} 1$, entry 12$)$ by complex 2 at R.T. 

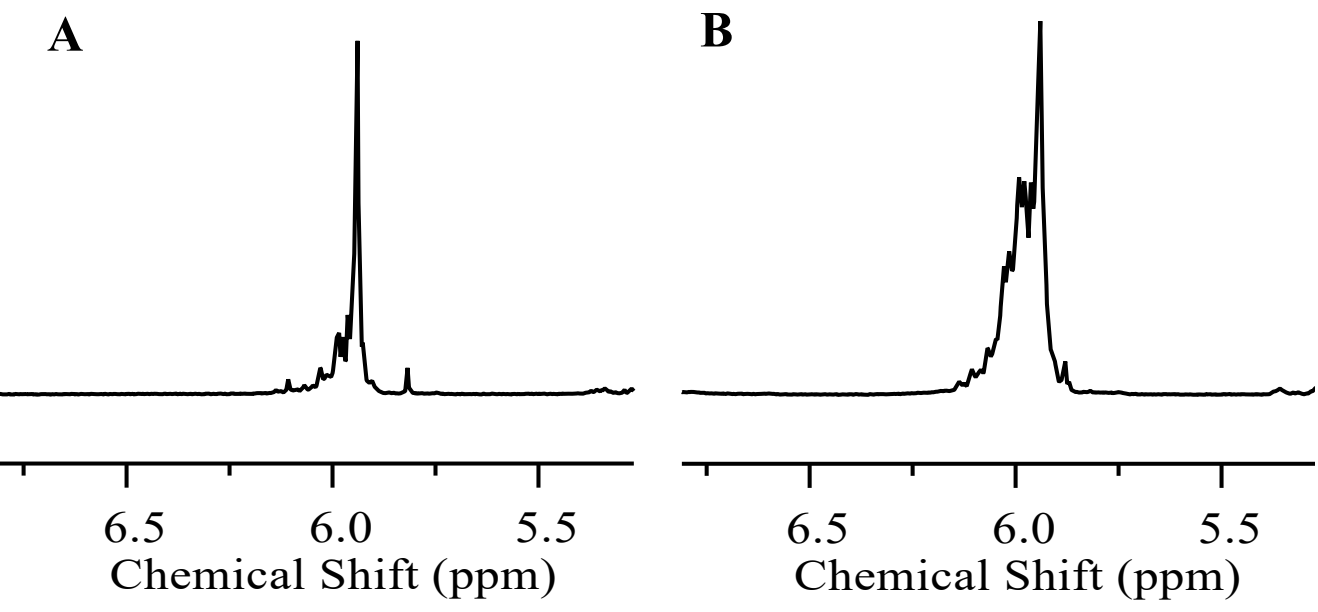

Figure S29. The methine resonances in ${ }^{1} \mathrm{H}$ NMR spectra of poly $($ rac-manOCA) obtained by ROP of rac-man $\mathrm{OCA}$ at the ratio of $\mathrm{A}:[\mathrm{Zn}]_{0} /[3-\mathrm{F}-\mathrm{Py}]_{0} /[\mathrm{L}-\mathrm{ML}]_{0} /[\mathrm{OCA}]_{0}(1 / 1 / 1 / 50)$ (Table 2 , entry 3$)$; $\mathrm{B}$ : $[\mathrm{Zn}]_{0} /[3-\mathrm{F}-\mathrm{Py}]_{0} /[\mathrm{L}-\mathrm{ML}]_{0} /[\mathrm{OCA}]_{0}(1 / 8 / 1 / 50)$ (Table $\mathrm{S} 1$, entry13) by complex 1 at $-30{ }^{\circ} \mathrm{C}$. 
Table S2. The ring-opening polymerization of ${ }_{\mathrm{L}}$-manOCA by $1 .^{a}$

\begin{tabular}{|c|c|c|c|c|}
\hline Entry & Time (min) & Conv. ${ }^{b}(\%)$ & $M_{\mathrm{n}, \mathrm{obsd}}{ }^{c}(\mathrm{~g} / \mathrm{mol})$ & 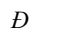 \\
\hline 1 & 5.5 & 17 & - & - \\
\hline 2 & 10 & 26 & 4000 & 1.09 \\
\hline 3 & 15.5 & 34 & 4700 & 1.12 \\
\hline 4 & 20 & 40 & 5400 & 1.12 \\
\hline 5 & 25.5 & 43 & 5800 & 1.12 \\
\hline 6 & 30 & 49 & 6100 & 1.11 \\
\hline 7 & 60 & 60 & 7200 & 1.12 \\
\hline 8 & 100 & 65 & 7800 & 1.11 \\
\hline 9 & 300 & 66 & 7700 & 1.12 \\
\hline 10 & 615 & 66 & 7800 & 1.12 \\
\hline
\end{tabular}

${ }^{a}$ Conditions: reactions were carried out under a dry nitrogen atmosphere at room temperature, $0.005 \mathrm{mmol}$ of complex $1,2 \mathrm{~mL}$ of toluene, $[\mathrm{Zn}]_{0} /[3-\mathrm{F}-\mathrm{Py}]_{0} /[\mathrm{L}-\mathrm{ML}]_{0} /[\mathrm{OCA}]_{0}=1: 1: 1: 100$. Take a small portion at regular intervals and measure the conversion. ${ }^{b}$ An aliquot was withdrawn to determine monomer conversion by ${ }^{1} \mathrm{H}$ NMR spectroscopy. ${ }^{c}$ Experimental $M_{\mathrm{n}}$ and $\varnothing$ determined by GPC in THF against polystyrene standards are not corrected.

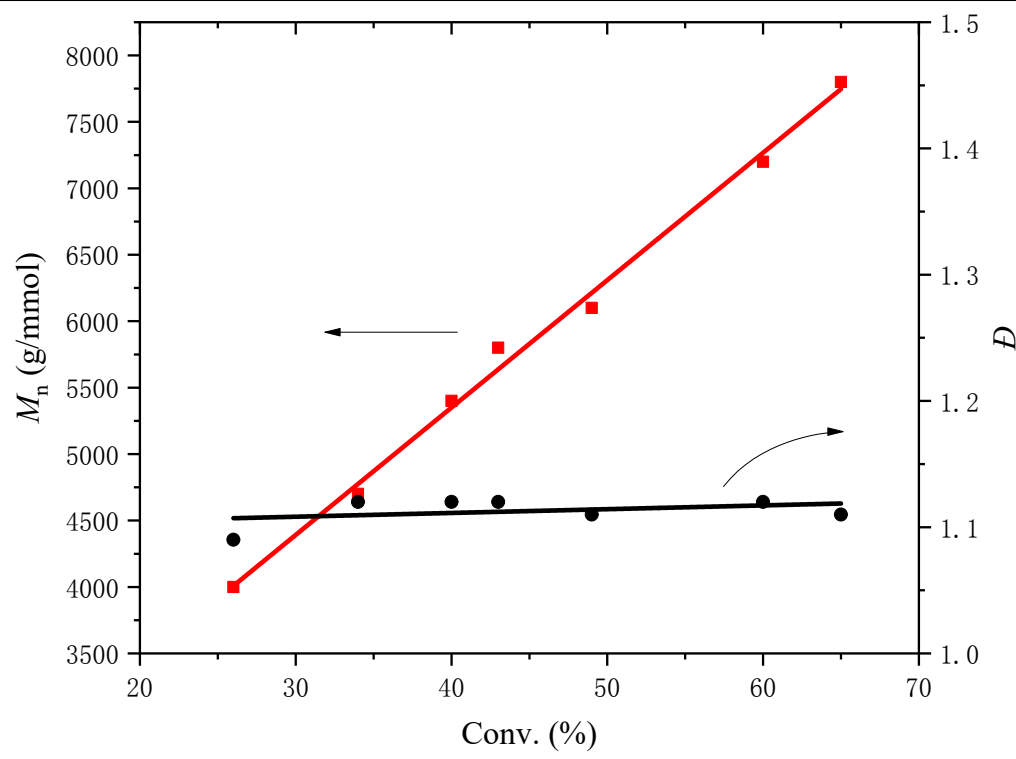

Figure S30. The relationship between $M_{\mathrm{n}}$ (Square), ( $($ (Circle) of the polymer vs conversion by table S2. 

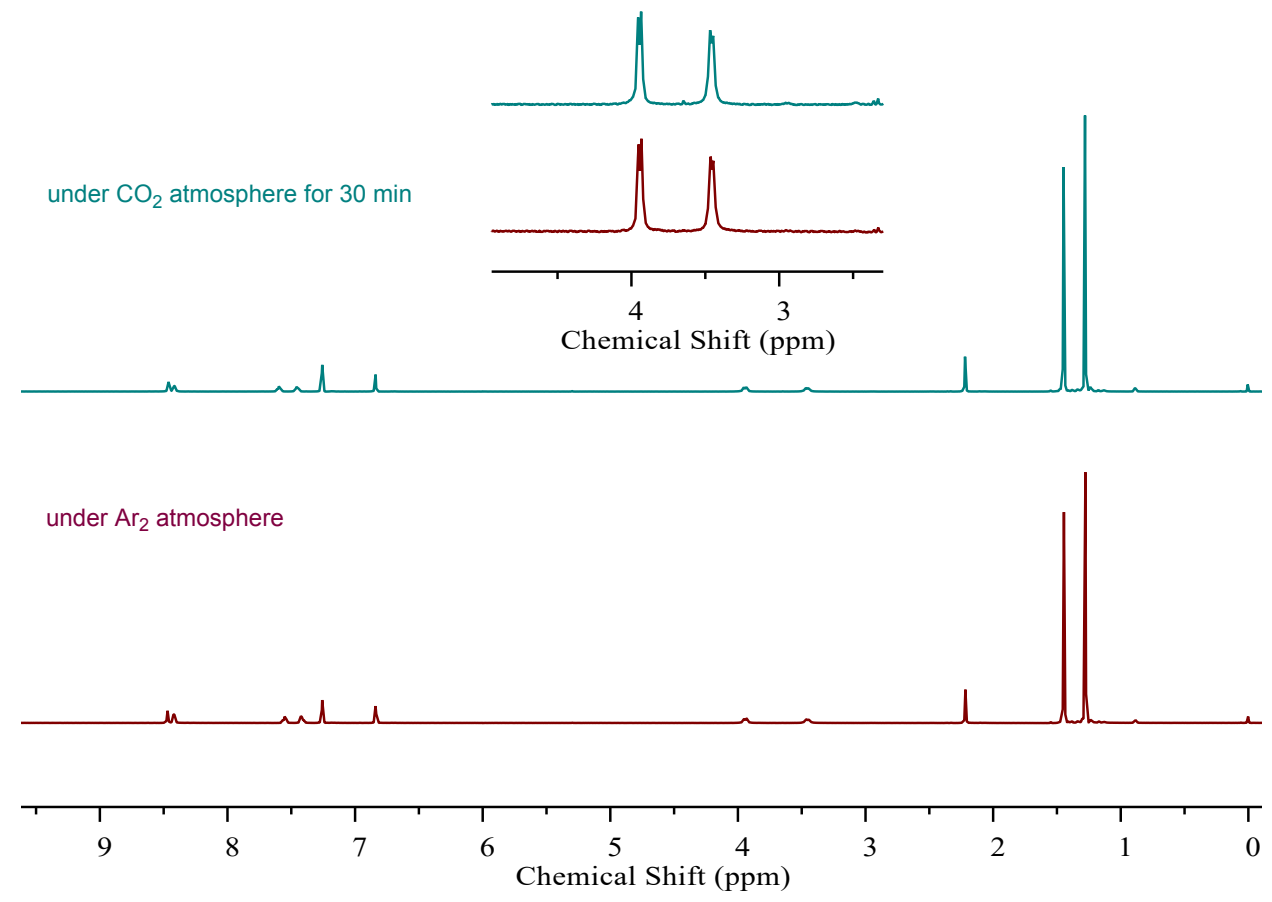

Figure S31. The ${ }^{1} \mathrm{H}$ NMR spectrum $\left(\mathrm{CDCl}_{3}, 600 \mathrm{M}\right)$ of $\mathbf{L}^{1} \mathbf{Z n}-3-\mathrm{F}-\mathrm{Pyz}$ under $\mathrm{CO}_{2}$ atmosphere for 30 min (top) and under $\mathrm{Ar}_{2}$ atmosphere (bottom). 
Table S3. The ring-opening polymerization of $\mathrm{L}_{\mathrm{L}}$ manOCA by 1 .

\begin{tabular}{|c|c|c|c|c|c|c|c|}
\hline Entry & {$[1]$} & [3-F-Py] & [L-ML] & Time (h) & Conv. $^{b}(\%)$ & $\begin{array}{c}M_{\mathrm{n}, \mathrm{obsd}}{ }^{c} \\
(\mathrm{~g} / \mathrm{mol})\end{array}$ & 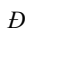 \\
\hline \multirow[t]{2}{*}{1} & 1 & 1 & 1 & 1 & 65 & 8400 & 1.15 \\
\hline & & & & 0.5 & 68 & 8500 & 1.17 \\
\hline \multirow[t]{2}{*}{2} & 1 & 1 & 1 & $1 \mathrm{~h}$ & 64 & 8500 & 1.15 \\
\hline & & +1 & & 0.5 & 66 & 8600 & 1.16 \\
\hline \multirow[t]{2}{*}{3} & 1 & 1 & 1 & $1 \mathrm{~h}$ & 70 & 8500 & 1.17 \\
\hline & +1 & & & 0.5 & 99 & 11000 & 1.17 \\
\hline
\end{tabular}

${ }^{a}$ Conditions: reactions were carried out under a dry nitrogen atmosphere at room temperature, $0.005 \mathrm{mmol}$ of $\mathbf{1}, 2$ of $\mathrm{mL}$ toluene, $[\mathrm{Zn}]_{0} /[3-\mathrm{F}-\mathrm{Py}]_{0} /[\mathrm{L}-\mathrm{ML}]_{0} /[\mathrm{OCA}]=1: 1: 1: 100$. After the polymerization was continued for 1 hour, the conversion of each reaction was measured. Then entry 1 was used as a blank, entry 2 was added with one equivalent of 3-f-Py, entry 3 was added with one equivalent of [Zn], and then the reaction was continued for another 30 minutes. ${ }^{b}$ Determined by ${ }^{1} \mathrm{H}$ NMR spectra. ${ }^{c}$ Experimental $M_{\mathrm{n}}$ and $\oslash$ determined by GPC in THF against polystyrene standards are not corrected.

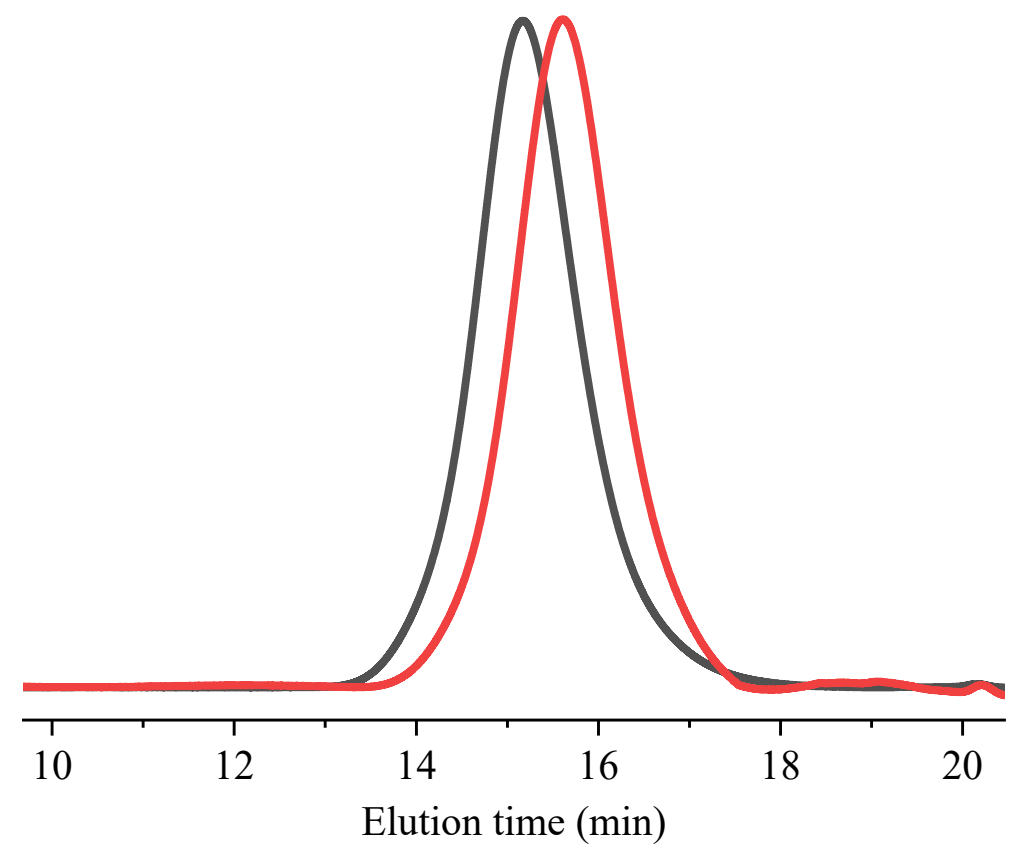

Figure S32. Gel-permeation chromatography (GPC) traces of the poly $(\mathrm{L}-$ manOCA) $\mathrm{t}=1 \mathrm{~h}(\mathrm{red}) ; \mathrm{t}=$ $1.5 \mathrm{~h}$ (black). (Table S3, entry 3). 

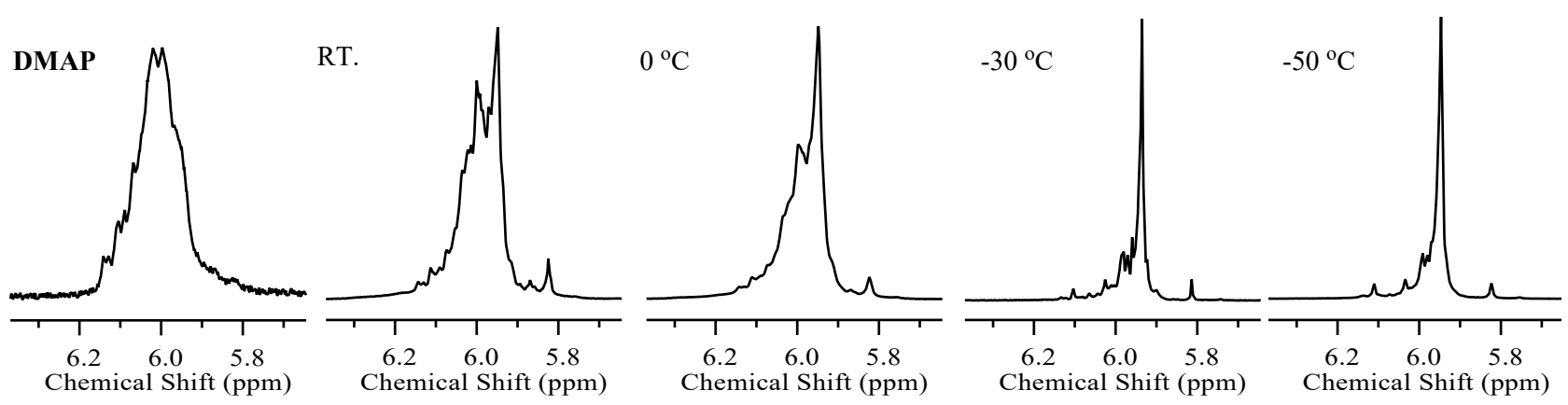

Figure S33. The methine resonances in ${ }^{1} \mathrm{H}$ NMR spectra of poly(rac-manOCA) via the ROP of rac-man $\mathrm{OCA}$ by Cat. 1 at $\mathrm{RT}$; $0{ }^{\circ} \mathrm{C} ;-30{ }^{\circ} \mathrm{C}$; $-50{ }^{\circ} \mathrm{C}$, respectively, as a comparison: poly $(r a c-m a n \mathrm{OCA})$ via the ROP of rac-manOCA by DMAP at R.T.

Cat. 1
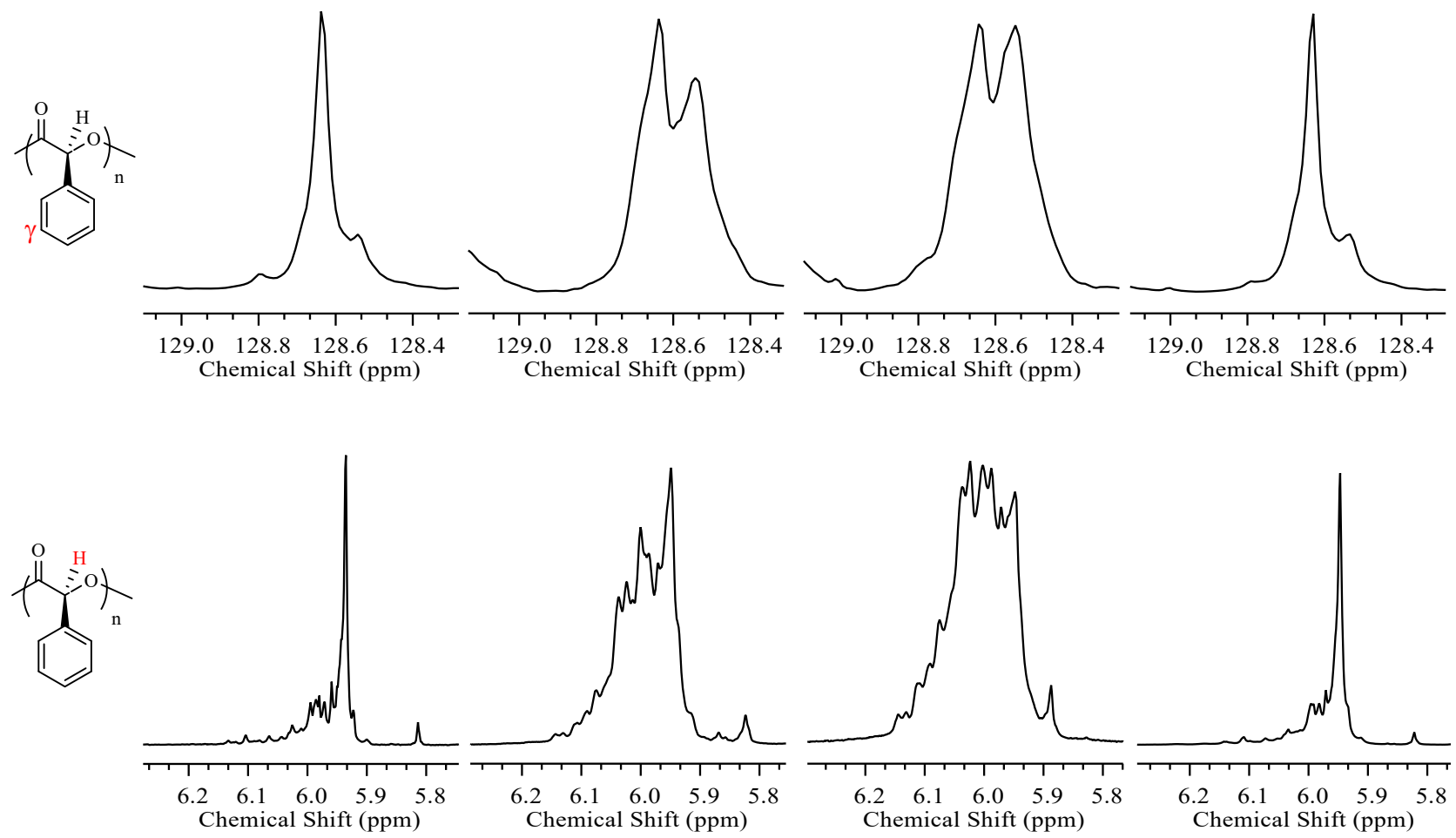

Figure S34. The $\gamma$-carbon of phenyl group resonances in ${ }^{13} \mathrm{C}$ NMR spectra (top) and the methine resonances in ${ }^{1} \mathrm{H}$ NMR spectra (bottom) of poly(rac-manOCA) via ROP of rac-manOCA by catalysts $1,2,4,5$ at $-30^{\circ} \mathrm{C}$, respectively. 
Table S4. Polymerizations of manOCA by 1 using different chiral methyl mandelates as initiators ${ }^{a}$.

\begin{tabular}{|c|c|c|c|c|c|c|c|c|c|}
\hline Entry & {$[\mathrm{OCA}]$} & $\begin{array}{l}\text { [configuration of } \\
\text { methyl } \\
\text { mandelate] }\end{array}$ & $\mathrm{T}\left({ }^{\circ} \mathrm{C}\right)$ & $\begin{array}{c}\text { Time } \\
(\mathrm{min})\end{array}$ & $\begin{array}{l}\text { Conv. }{ }^{b} \\
(\%)\end{array}$ & $\begin{array}{c}M_{\mathrm{n}, \mathrm{obsd}}{ }^{c} \\
(\mathrm{~g} / \mathrm{mol})\end{array}$ & $\begin{array}{l}M_{\mathrm{n}, \mathrm{NMR}}{ }^{d}(\mathrm{~g} / \\
\mathrm{mol})\end{array}$ & $\begin{array}{c}M_{\mathrm{n}, \text { calcd }}{ }^{2} \\
(\mathrm{~g} / \mathrm{mol})\end{array}$ & $Ð$ \\
\hline 1 & $\mathrm{~L}-\operatorname{man} \mathrm{OCA}$ & L- & RT & 30 & 85 & 5900 & 5600 & 5900 & 1.09 \\
\hline 2 & $\mathrm{D}^{-m a n O C A}$ & L- & RT & 30 & 90 & 6600 & 6400 & 6000 & 1.10 \\
\hline 3 & $\mathrm{~L}-\operatorname{man} \mathrm{OCA}$ & rac & RT & 30 & 89 & 5700 & 5600 & 6000 & 1.09 \\
\hline 4 & rac-man $\mathrm{OCA}$ & $\mathrm{rac}$ & RT & 30 & 82 & 5400 & 5600 & 5700 & 1.10 \\
\hline 5 & rac-man $\mathrm{OCA}$ & rac & 0 & $2 \mathrm{~h}$ & 80 & 4900 & 5000 & 5500 & 1.11 \\
\hline 6 & rac-man $\mathrm{OCA}$ & rac & -30 & $9 \mathrm{~h}$ & 94 & 6100 & 6000 & 6400 & 1.08 \\
\hline 7 & rac-man $\mathrm{OCA}$ & rac & -50 & $9.5 \mathrm{~h}$ & 79 & 5300 & 5000 & 5300 & 1.12 \\
\hline $8^{f}$ & rac-manOCA & $\mathrm{rac}$ & RT & 5 & 99 & 3100 & 6000 & 6700 & 1.17 \\
\hline
\end{tabular}

${ }^{a}$ Conditions: reactions were carried out under a dry nitrogen atmosphere, $0.01 \mathrm{mmol}$ of $[\mathrm{Zn}]_{\mathrm{o}}$, [Initiator] $=$ methyl mandelate, $[\mathrm{Zn}]=0.01 \mathrm{M}, 2 \mathrm{~mL}$ Toluene, $[\mathrm{Zn}]_{0} /[3-\mathrm{F}-\mathrm{Py}]_{0} /[\text { Initiator }]_{0} /[\mathrm{OCA}]=1: 1: 1: 50 .{ }^{b}$ Determined by ${ }^{1} \mathrm{H}$ NMR spectrum. ${ }^{c}$ Experimental $M_{\mathrm{n}}$ and $\doteq$ determined by GPC in THF against polystyrene standards are not corrected. ${ }^{d}$ Determined from the relative integration of the signals for the main-chain methine units and chain ends. ${ }^{e}$ Calculated from the equation (molar mass of $\mathrm{OCA}-$ molar mass of $\left.\mathrm{CO}_{2}\right) \times[\mathrm{OCA}]_{0} /[\text { Initiator }]_{0} \times$ Conversion $\%+$ the molar mass of initiator. $f \mathrm{ROP}$ of $\mathrm{rac}$-manOCA by DMAP.

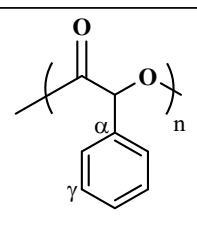

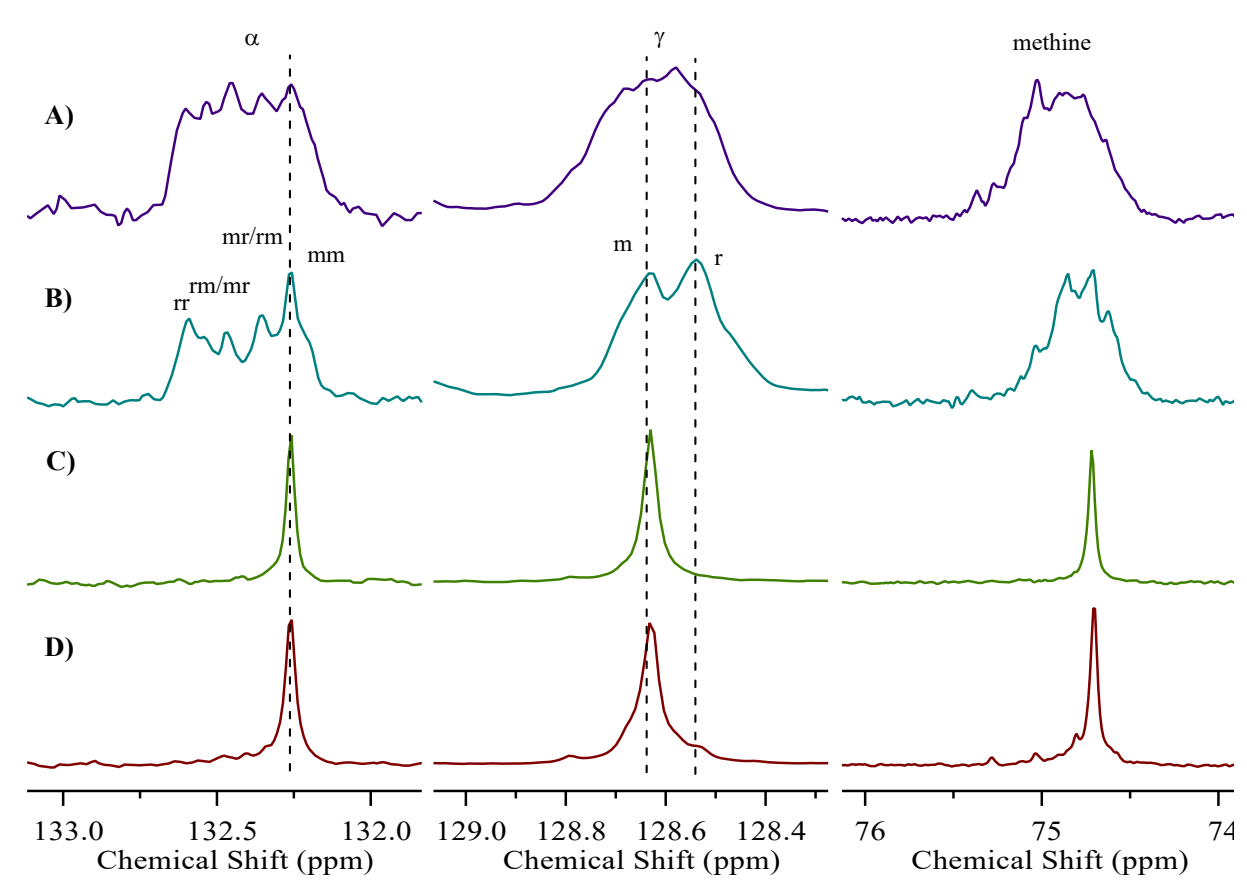

Figure $\mathrm{S} 35$. The ${ }^{13} \mathrm{C}$ NMR spectra (100 $\left.\mathrm{MHz}, \mathrm{CDCl}_{3}\right)$ of poly (manOCA)s synthesized via A) ROP of rac-man OCA by DMAP (Table S4, entry 8), B) ROP of rac-manOCA by complex 1 at RT. (Table S4, entry 4), C) ROP of ${ }_{L}-$ manOCA by 1 at RT. (Table S4, entry 3) and D) ROP of rac-man $\mathrm{OCA}$ by complex 1 at $-50^{\circ} \mathrm{C}$. (Table $\mathrm{S} 4$, entry 7 ). 

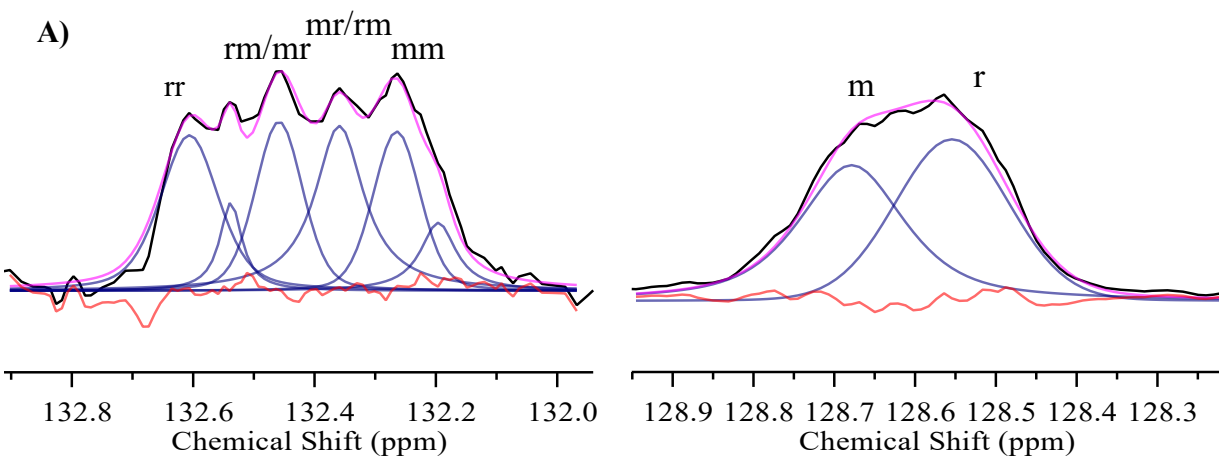

128.9128 .8128 .7128 .6128 .5128 .4128 .3

Chemical Shift (ppm)
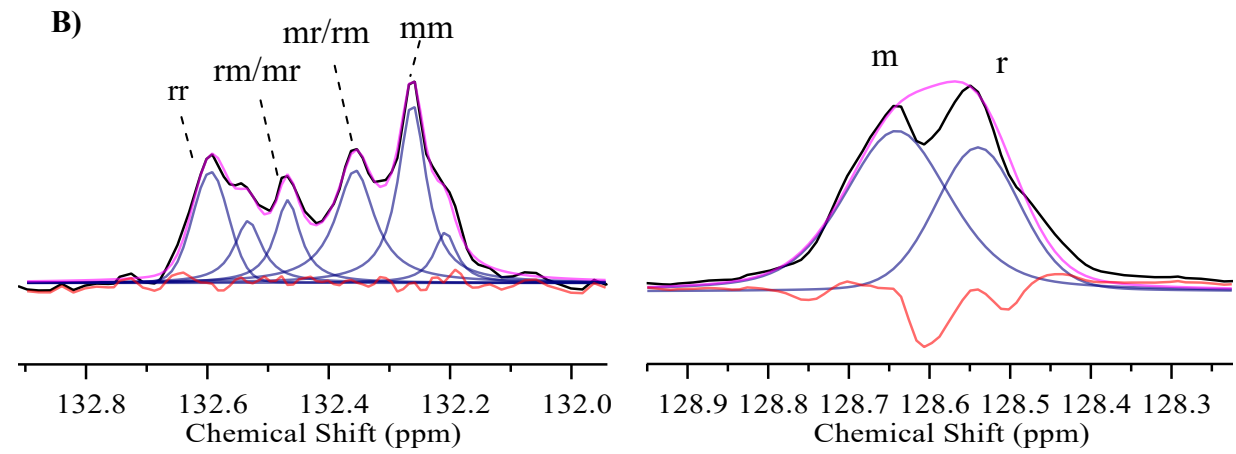

C)
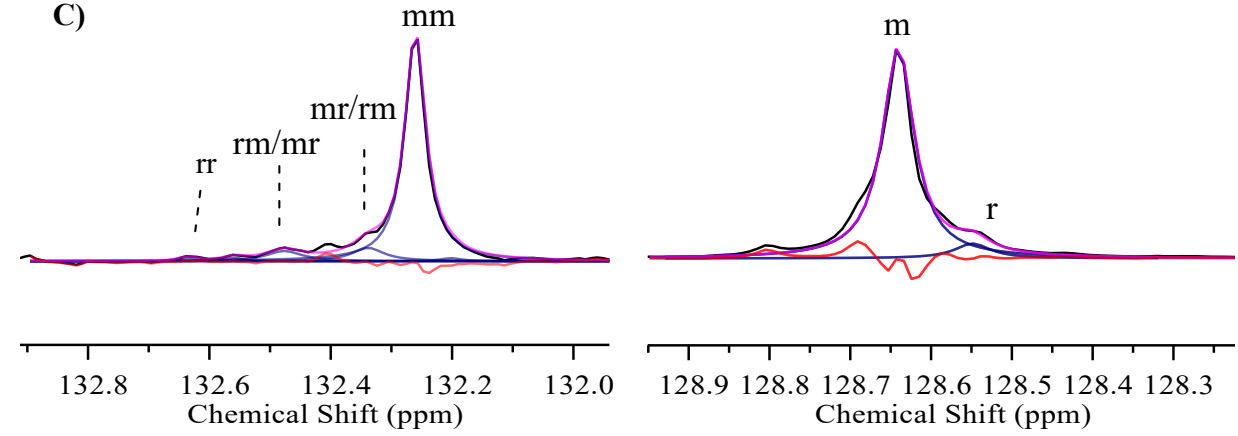

Figure S36. Regions of the main-chain $\alpha$-carbon of phenyl (left) and $\gamma$-carbon of phenyl (right) in the ${ }^{13} \mathrm{C}$ NMR spectra of poly (manOCA) from the ROP of rac-manOCA by A) DMAP (Table S4, entry 8), B) complex 1 at RT (Table S4, entry 4) and C) complex 1 at $-50{ }^{\circ} \mathrm{C}$ (Table S4, entry 7) 
Table S5. Relative stereosequence proportions in atactic poly(rac-manOCA) resulting from the ROP of rac-manOCA with DMAP (Table S4, entry 8).

\begin{tabular}{|c|c|c|c|c|c|}
\hline \multirow[t]{2}{*}{ Assignment } & \multirow[t]{2}{*}{ Probability } & \multicolumn{2}{|l|}{$\alpha$} & \multicolumn{2}{|l|}{$\gamma$} \\
\hline & & Experimental probability & $P_{\mathrm{m}}$ & Experimental probability & $P_{\mathrm{m}}$ \\
\hline Triad $\mathrm{mm}$ & $P_{\mathrm{m}}^{2}$ & $0.176, \quad 0.077$ & 0.50 & \multirow[t]{2}{*}{0.50} & \multirow[t]{2}{*}{0.50} \\
\hline $\mathrm{mr} / \mathrm{rm}$ & $P_{\mathrm{m}}{ }^{*} P_{\mathrm{r}}$ & 0.255 & 0.50 & & \\
\hline $\mathrm{rm} / \mathrm{mr}$ & $P_{\mathrm{m}}{ }^{*} P_{\mathrm{r}}$ & $0.190, \quad 0.062$ & 0.50 & \multirow[t]{2}{*}{0.50} & \multirow[t]{2}{*}{0.50} \\
\hline $\mathrm{rr}$ & $P_{\mathrm{r}}^{2}$ & 0.241 & 0.49 & & \\
\hline
\end{tabular}

Table S6. Relative stereosequence proportions in at poly(rac-manOCA) resulting from the ROP of rac-manOCA with complex 1 as a catalyst at RT. (Table S4, entry 4).

\begin{tabular}{|c|c|c|c|c|c|}
\hline \multirow[t]{2}{*}{ Assignment } & \multirow[t]{2}{*}{ Probability } & \multicolumn{2}{|l|}{$\alpha$} & \multicolumn{2}{|l|}{$\gamma$} \\
\hline & & Experimental probability & $P_{\mathrm{m}}$ & Experimental probability & $P_{\mathrm{m}}$ \\
\hline Triad $\mathrm{mm}$ & $P_{m}^{2}$ & $0.358(0.291,0.067)$ & 0.60 & \multirow[t]{2}{*}{0.61} & \multirow[t]{2}{*}{0.61} \\
\hline $\mathrm{mr} / \mathrm{rm}$ & $P_{m} * P_{r}$ & 0.256 & 0.58 & & \\
\hline $\mathrm{rm} / \mathrm{mr}$ & $P_{m}{ }^{*} P_{r}$ & $(0.123,0.102)$ & 0.61 & \multirow[t]{2}{*}{0.39} & \multirow[t]{2}{*}{0.39} \\
\hline $\mathrm{rr}$ & $P_{r}^{2}$ & 0.162 & 0.60 & & \\
\hline
\end{tabular}

Table S7. Relative stereosequence proportions in poly(rac-man OCA) resulting from the ROP of rac-manOCA with complex $\mathbf{1}$ as a catalyst at -50 ${ }^{\circ} \mathrm{C}$ (Table S4, entry 7).

\begin{tabular}{|c|c|c|c|c|c|}
\hline \multirow[t]{2}{*}{ Assignment } & \multirow[t]{2}{*}{ Probability } & \multicolumn{2}{|l|}{$\alpha$} & \multicolumn{2}{|l|}{$\gamma$} \\
\hline & & Experimental probability & $P_{\mathrm{m}}$ & Experimental probability & $P_{\mathrm{m}}$ \\
\hline Triad $\mathrm{mm}$ & $P_{m}{ }^{2}$ & 0.852 & 0.92 & \multirow[t]{2}{*}{0.924} & \multirow[t]{2}{*}{0.92} \\
\hline $\mathrm{mr} / \mathrm{rm}$ & $P_{m}{ }^{*} P_{r}$ & 0.0798 & 0.92 & & \\
\hline $\mathrm{rm} / \mathrm{mr}$ & $P_{m} * P_{r}$ & 0.0686 & 0.93 & \multirow[t]{2}{*}{0.076} & \multirow[t]{2}{*}{0.08} \\
\hline $\mathrm{rr}$ & $P_{r}^{2}$ & - & - & & \\
\hline
\end{tabular}




\section{Analysis chain end regularity}

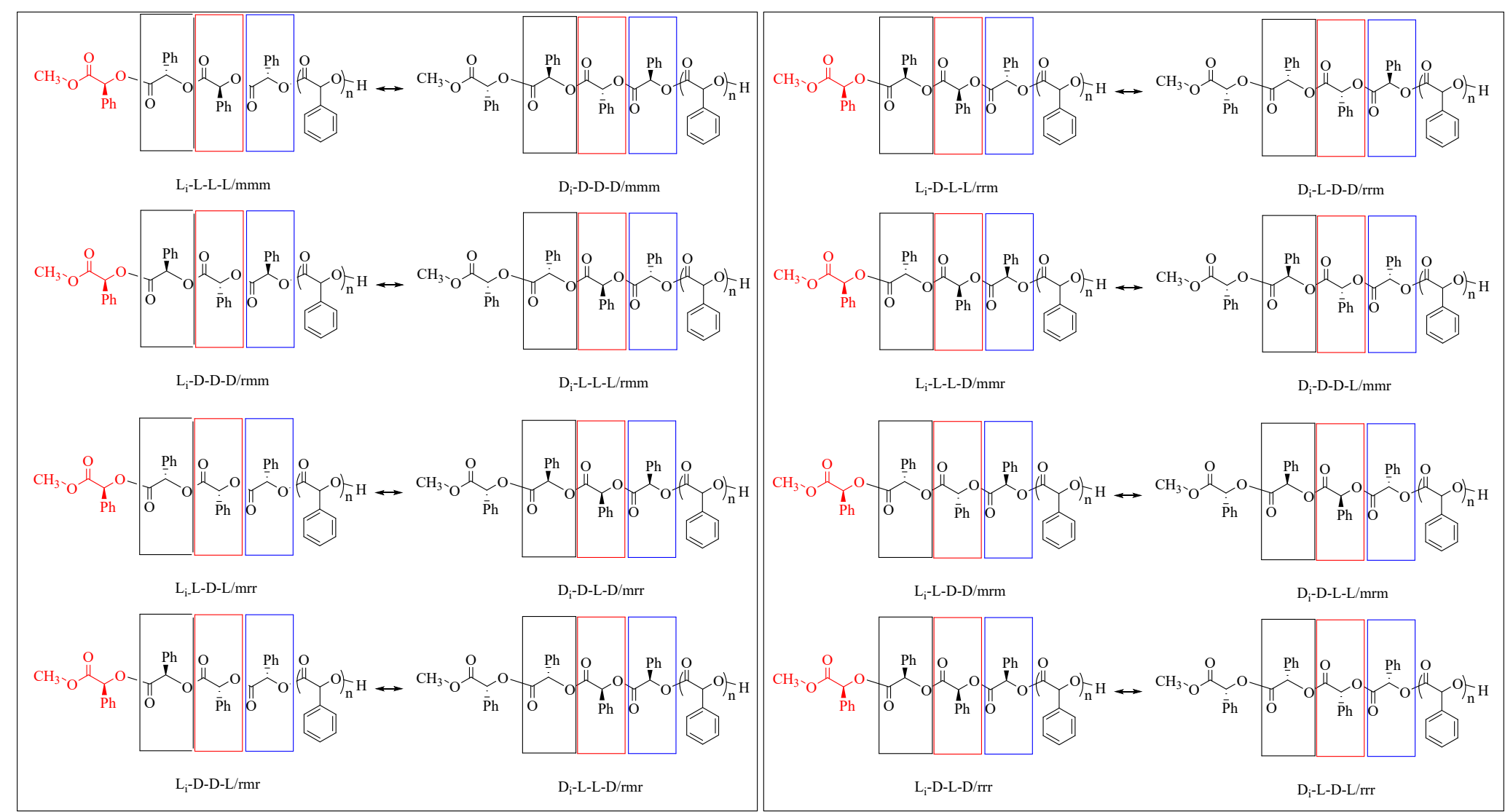

Figure S37. Structural formula of different tetrads in chain end of poly(manOCA). 


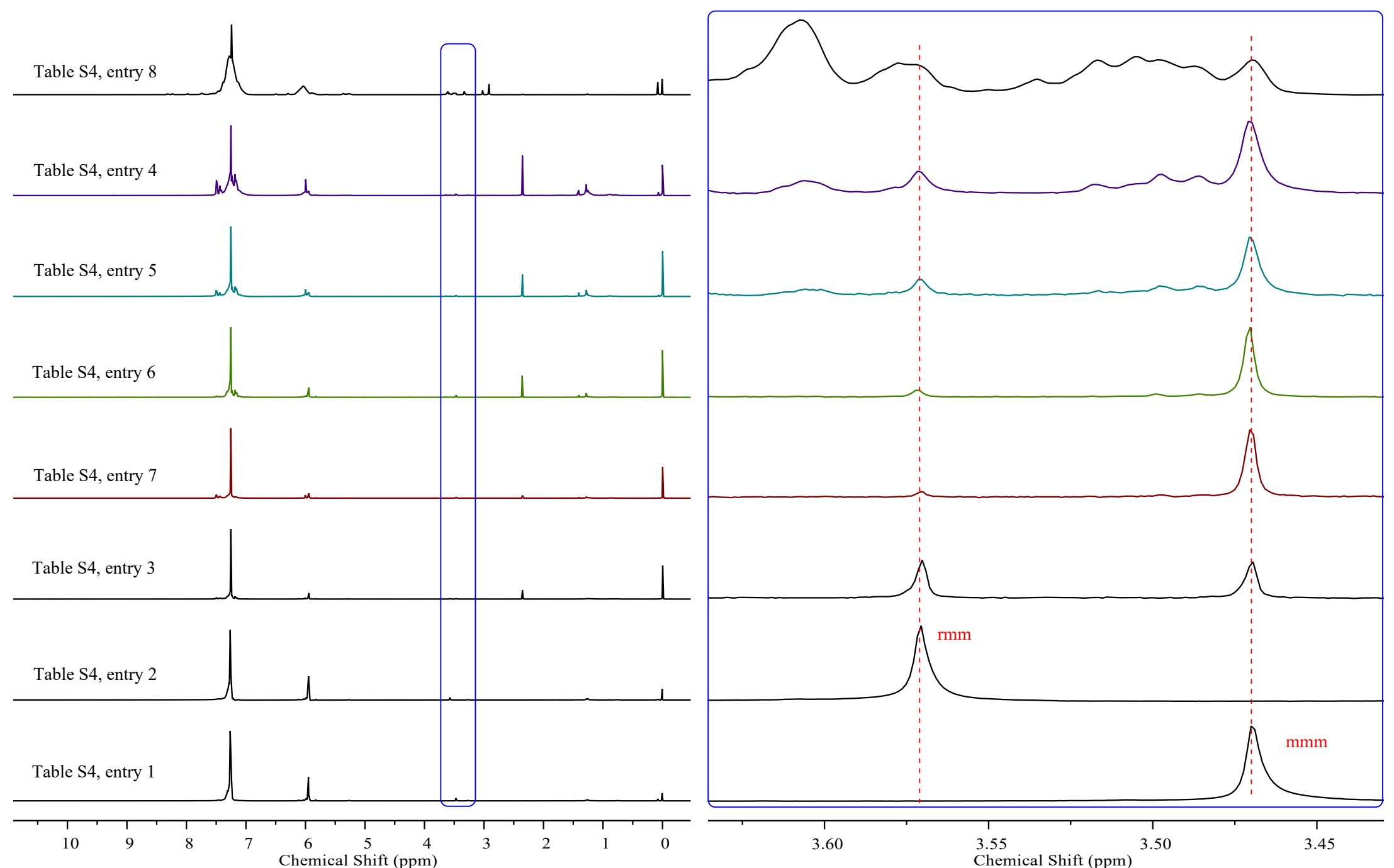

Figure S38. ${ }^{1} \mathrm{H}$ NMR spectra of methoxy region in the chain end of polymers obtained via different ROPs using different configuration of initiators in Table S4. 
A)

$$
\mathrm{mmm}, \mathrm{rrm} / \mathrm{mrr} / \mathrm{rmr}
$$

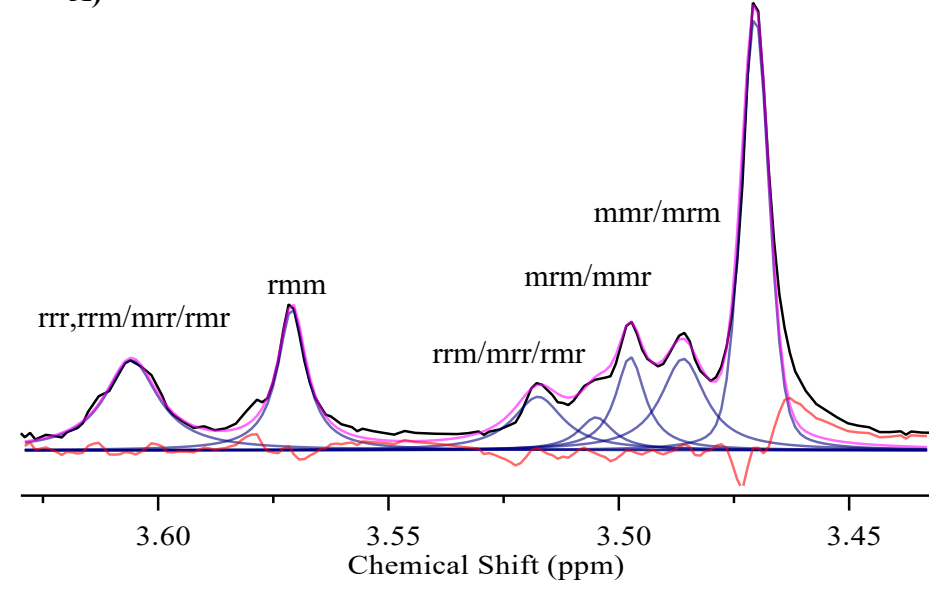

B)

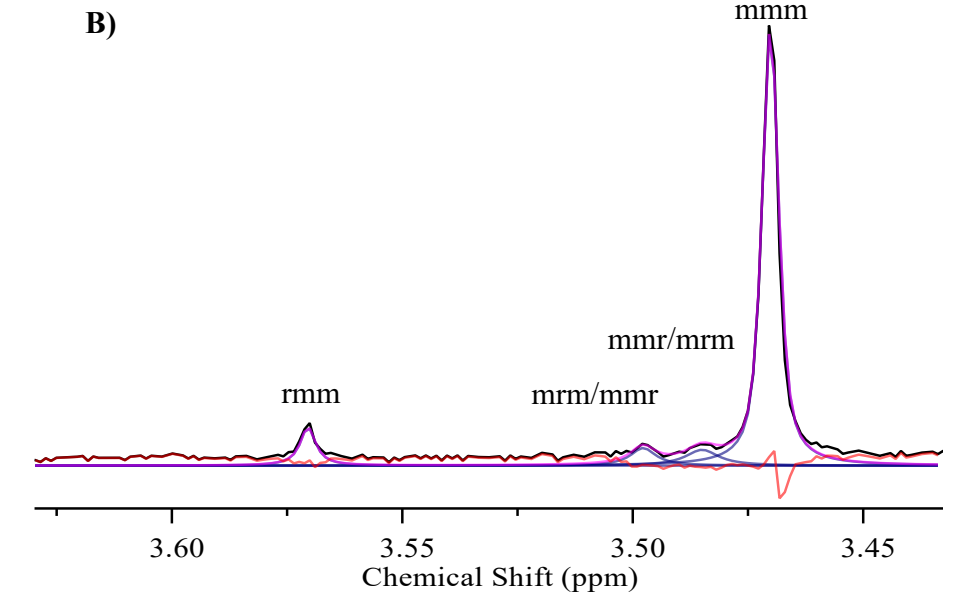

Figure S39. Regions of the methoxy in the ${ }^{1} \mathrm{H}$ NMR spectra of poly(manOCA) obtained from the ROP of rac-manOCA by a) complex 1 at RT (Table S4, entry 3) and b) complex 1 at $-50{ }^{\circ} \mathrm{C}$ (Table S4, entry 7).

Table S8. Relative stereosequence proportions in poly(rac-manOCA) resulting from the ROP of rac-manOCA with complex $\mathbf{1}$ at RT.

\begin{tabular}{|c|c|c|c|}
\hline Assignment & Probability & Experimental probability & $P_{\mathrm{m}}$ \\
\hline Tetrad $\mathrm{mmm}$ & $P_{\mathrm{m}}^{3}$ & \multirow{2}{*}{0.322} & \multirow{2}{*}{0.61} \\
\hline $\mathrm{mrr}$ & $P_{\mathrm{m}} * P_{\mathrm{r}}^{2}$ & & \\
\hline $\mathrm{mmr}$ & $P_{\mathrm{m}}^{2 *} P_{\mathrm{r}}$ & 0.143 & 0.6 \\
\hline $\mathrm{mrm}$ & $P_{\mathrm{m}}^{2 *} P_{\mathrm{r}}$ & $0.096,0.04$ & 0.6 \\
\hline $\mathrm{rmr}$ & $P_{\mathrm{m}} * P_{\mathrm{r}}^{2}$ & 0.095 & 0.6 \\
\hline $\mathrm{rmm}$ & $P_{\mathrm{m}}^{2 *} P_{\mathrm{r}}$ & 0.142 & 0.6 \\
\hline $\mathrm{rrm}$ & $P_{\mathrm{m}} * P_{\mathrm{r}}^{2}$ & \multirow{2}{*}{0.162} & \multirow{2}{*}{0.6} \\
\hline $\operatorname{rrr}$ & $P_{\mathrm{r}}^{3}$ & & \\
\hline
\end{tabular}

Table S9. Relative stereosequence proportions in poly (rac-manOCA) resulting from the ROP of rac-manOCA with 1 at $-50{ }^{\circ} \mathrm{C}$

\begin{tabular}{|r|c|c|c|}
\hline \multicolumn{1}{|c|}{ Assignment } & Probability & Experimental probability & $P_{\mathrm{m}}$ \\
\hline Tetrad $\mathrm{mmm}$ & $P_{\mathrm{m}}{ }^{3}$ & 0.806 & 0.93 \\
\hline $\mathrm{mrr}$ & $P_{\mathrm{m}}{ }^{*} P_{\mathrm{r}}{ }^{2}$ & - & - \\
\hline $\mathrm{mmr}$ & $P_{\mathrm{m}}{ }^{2} P_{\mathrm{r}}$ & 0.056 & 0.93 \\
\hline
\end{tabular}




\begin{tabular}{|r|c|c|c|}
\hline $\mathrm{mrm}$ & $P_{\mathrm{m}}{ }^{2} P_{\mathrm{r}}$ & 0.065 & 0.93 \\
\hline $\mathrm{rmr}$ & $P_{\mathrm{m}}{ }^{2} P_{\mathrm{r}}$ & - & - \\
\hline $\mathrm{rmm}$ & $P_{\mathrm{m}}{ }^{2} P_{\mathrm{r}}$ & 0.073 & 0.92 \\
\hline $\mathrm{rrm}$ & $P_{\mathrm{m}}{ }^{2} P_{\mathrm{r}}^{2}$ & - & - \\
\hline $\mathrm{rrm}$ & $P_{\mathrm{r}}{ }^{3}$ & - & - \\
\hline
\end{tabular}
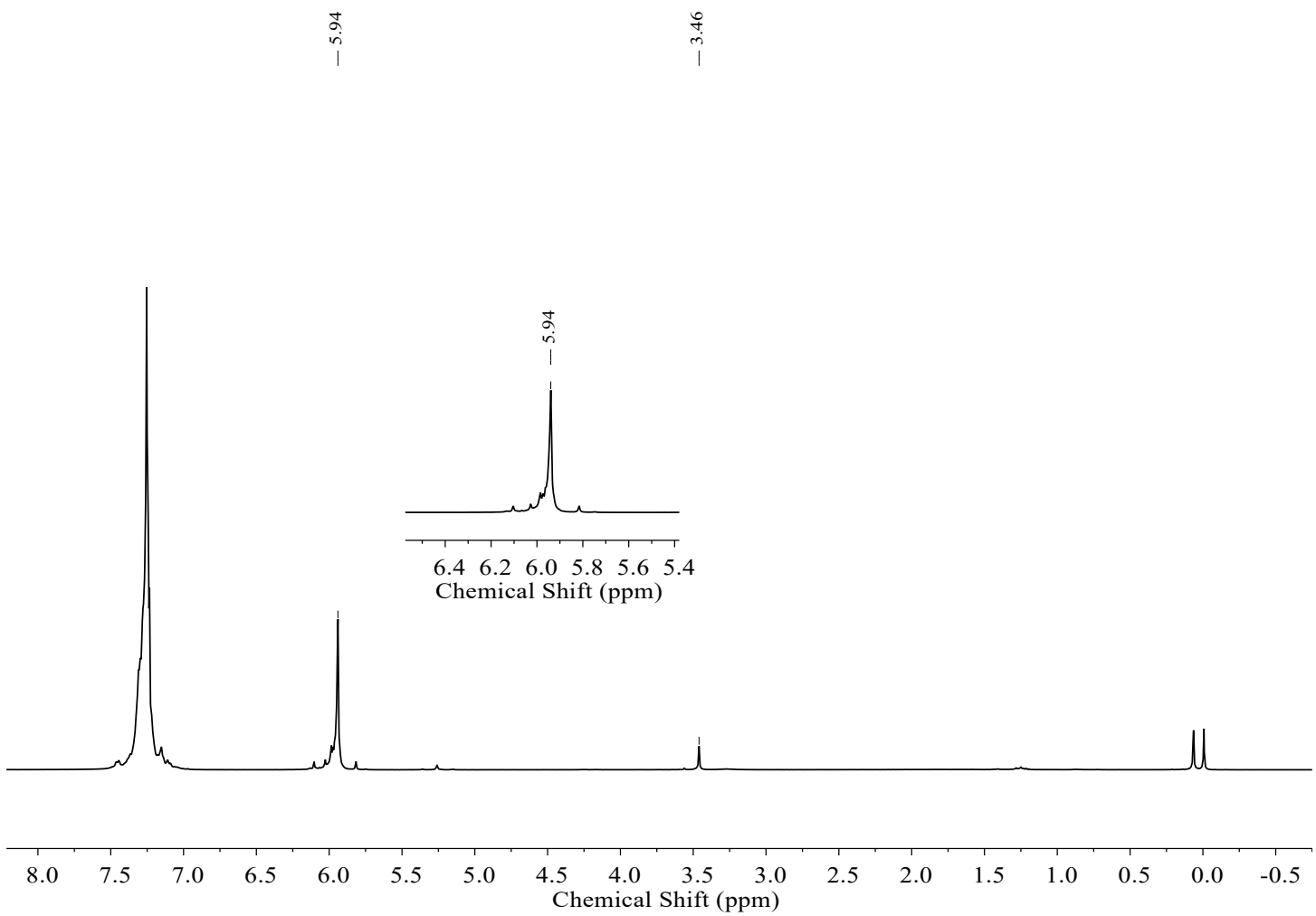

Figure S40. The ${ }^{1} \mathrm{H}$ NMR spectrum of poly(manOCA) $\left(400 \mathrm{M}, \mathrm{CDCl}_{3}\right)$ via the ROP of rac-man OCA using complex 1 as a catalyst at $-50{ }^{\circ} \mathrm{C}$ (Table 2 , entry 4$)$. 

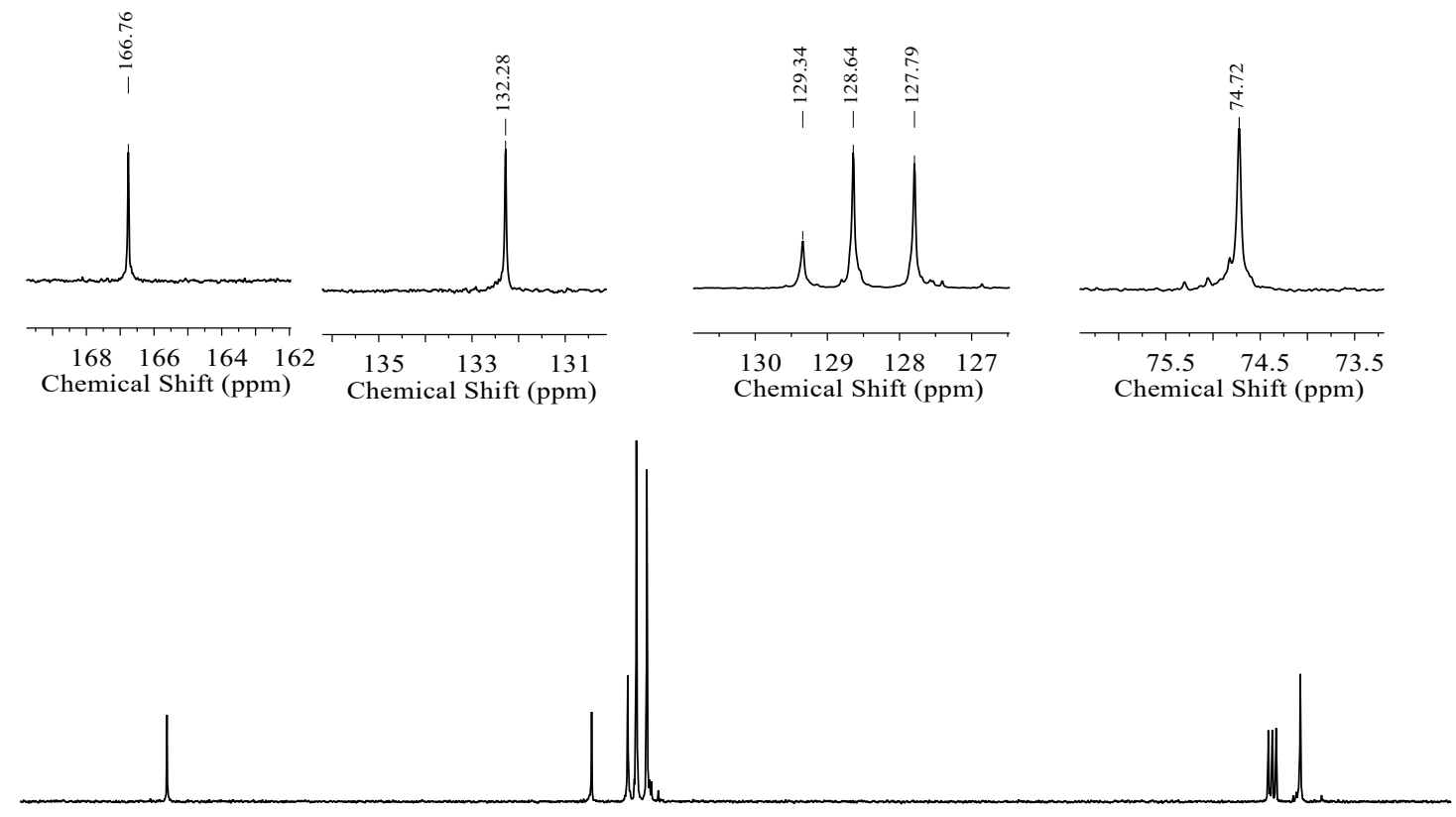

$\begin{array}{lllllllllllllllllllllll}175 & 170 & 165 & 160 & 155 & 150 & 145 & 140 & 135 & 130 & 125 & 120 & 115 & 110 & 105 & 100 & 95 & 90 & 85 & 80 & 75 & 70 & 65\end{array}$

Figure S41. The ${ }^{13} \mathrm{C}$ NMR spectrum of poly(manOCA) $\left(100 \mathrm{M}, \mathrm{CDCl}_{3}\right)$ via the $\mathrm{ROP}$ of rac-manOCA using complex 1 as a catalyst at $-50{ }^{\circ} \mathrm{C}$ (Table 2 , entry 4 ). 


\section{Crystal Structures}

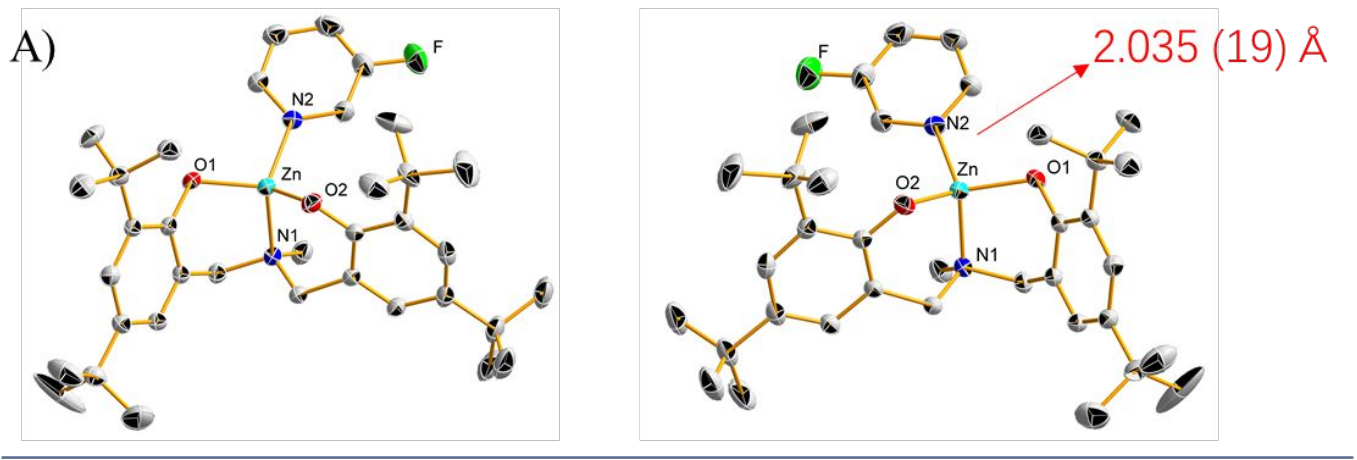

B)
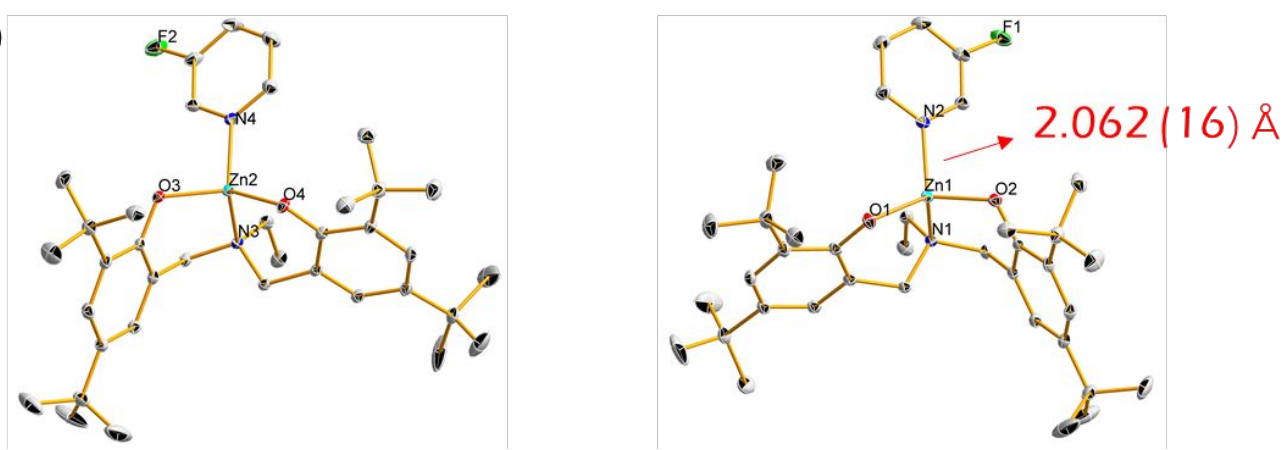

Figure S42. X-ray structures of $\mathbf{L}^{\mathbf{1}} \mathbf{Z n}+3$-F-Py (1899039) (a) and $\mathbf{L}^{2} \mathbf{Z n}+3-F-P y(1899031)$. (b) as $30 \%$ thermal ellipsoids with hydrogen atoms omitted for clarity. Selected bond distances $(\AA)$ for $\mathbf{L}^{1} \mathbf{Z n}+$ 3-F-Py: Zn1-O1, 1.9023(15); Zn1-O2, 1.8963(16); Zn1-N1, 2.0634(17); Zn1-N2, 2.0352(19). Selected bond distances $(\AA)$ for $\mathbf{L}^{2} \mathbf{Z n}+3-F-P y: Z n 1-O 1,1.8902(14)$; Zn1-O2, 1.8967(13); Zn1-N1, 2.0480(15); Zn1-N2, 2.0615(16); Zn2-O3 1.8975(13); Zn2-O4, 1.8924(13); Zn2-N3, 2.0497(15); Zn2-N4, 2.0587(16). 


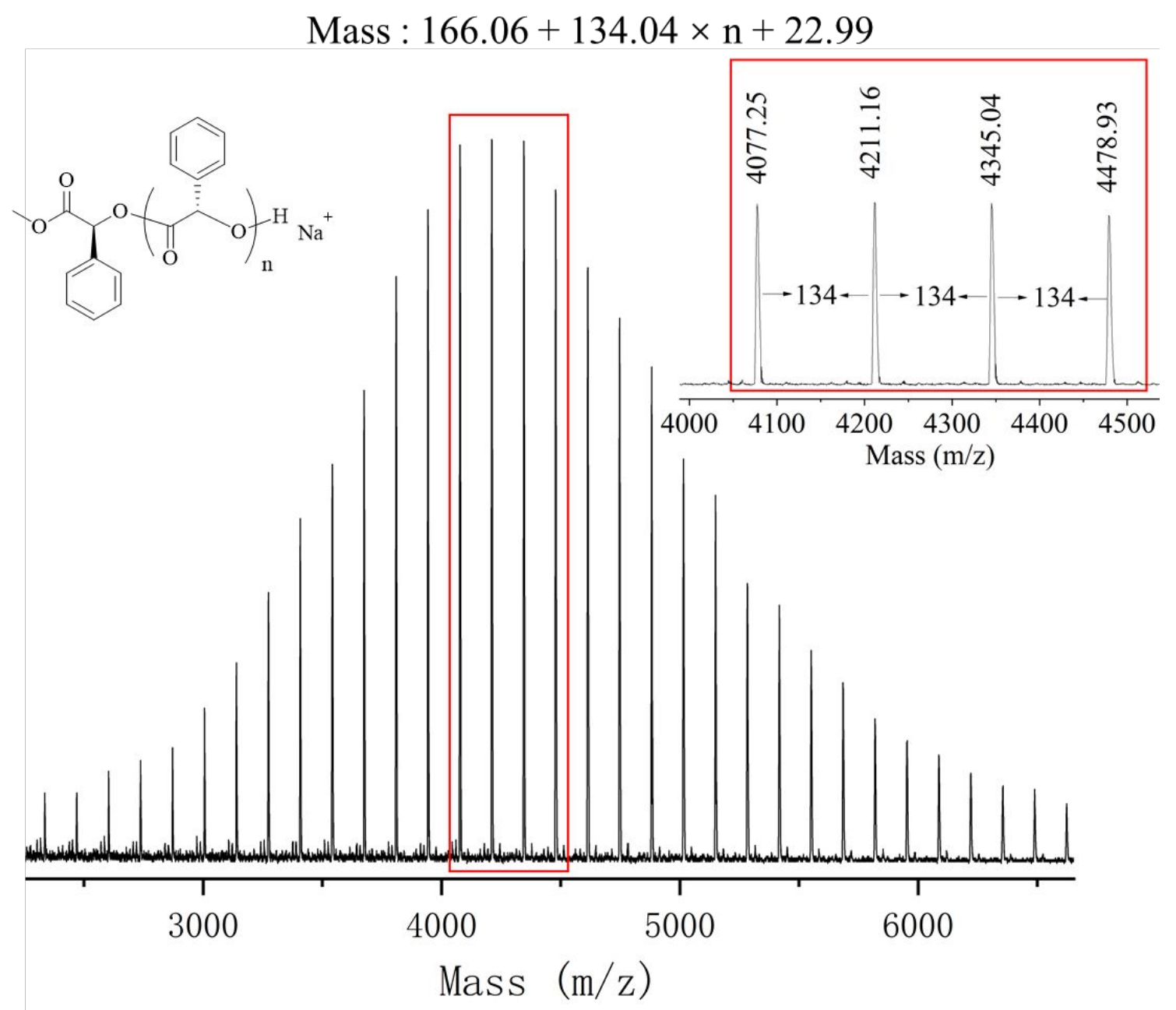

Figure S43. The MALDI-TOF mass spectrum of poly(L-manOCA) obtained using complex $\mathbf{1}$ as a catalyst (Table S4, entry 1). 


\section{Differential scanning calorimetry (DSC) and thermal gravimetric (TG).}

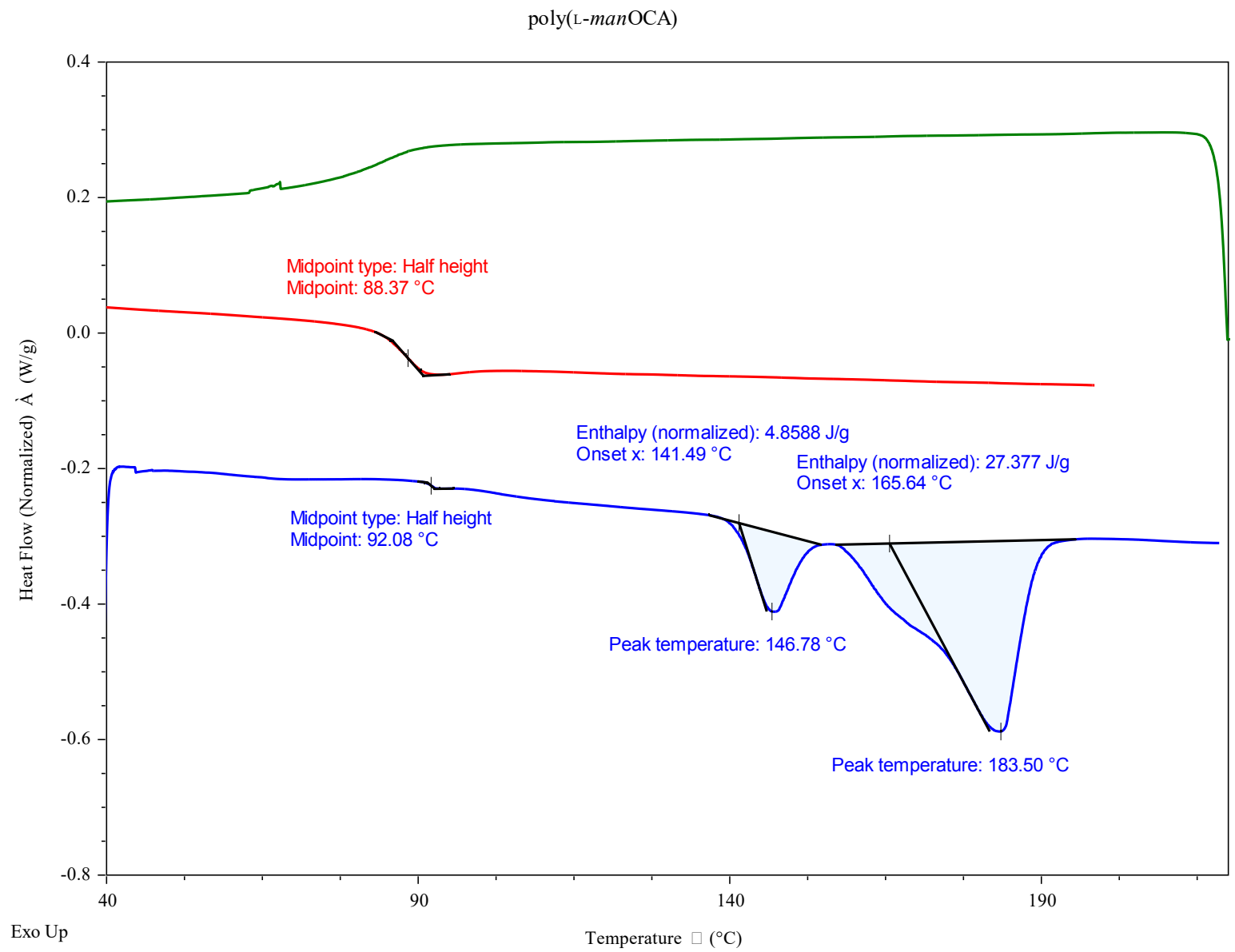

Figure S44. DSC curves of poly $\left({ }_{L}-\right.$ manOCA $)\left(M_{\mathrm{n}}=5300, D=1.10\right)$ produced via the ROP of $\mathrm{L}^{-}$-manOCA using complex 1 as a catalyst (Table 1, entry 1), $T_{\mathrm{m} 1}=147{ }^{\circ} \mathrm{C}$ (first heating scan); $T_{\mathrm{m} 2}=$ $184{ }^{\circ} \mathrm{C}$ (first heating scan); $T_{\mathrm{g}}=88^{\circ} \mathrm{C}$ (second heating scan) (Heating and cooling rate $10{ }^{\circ} \mathrm{C} / \mathrm{min}$, first heating: blue line, first cooling: green line, second heating: red line). 


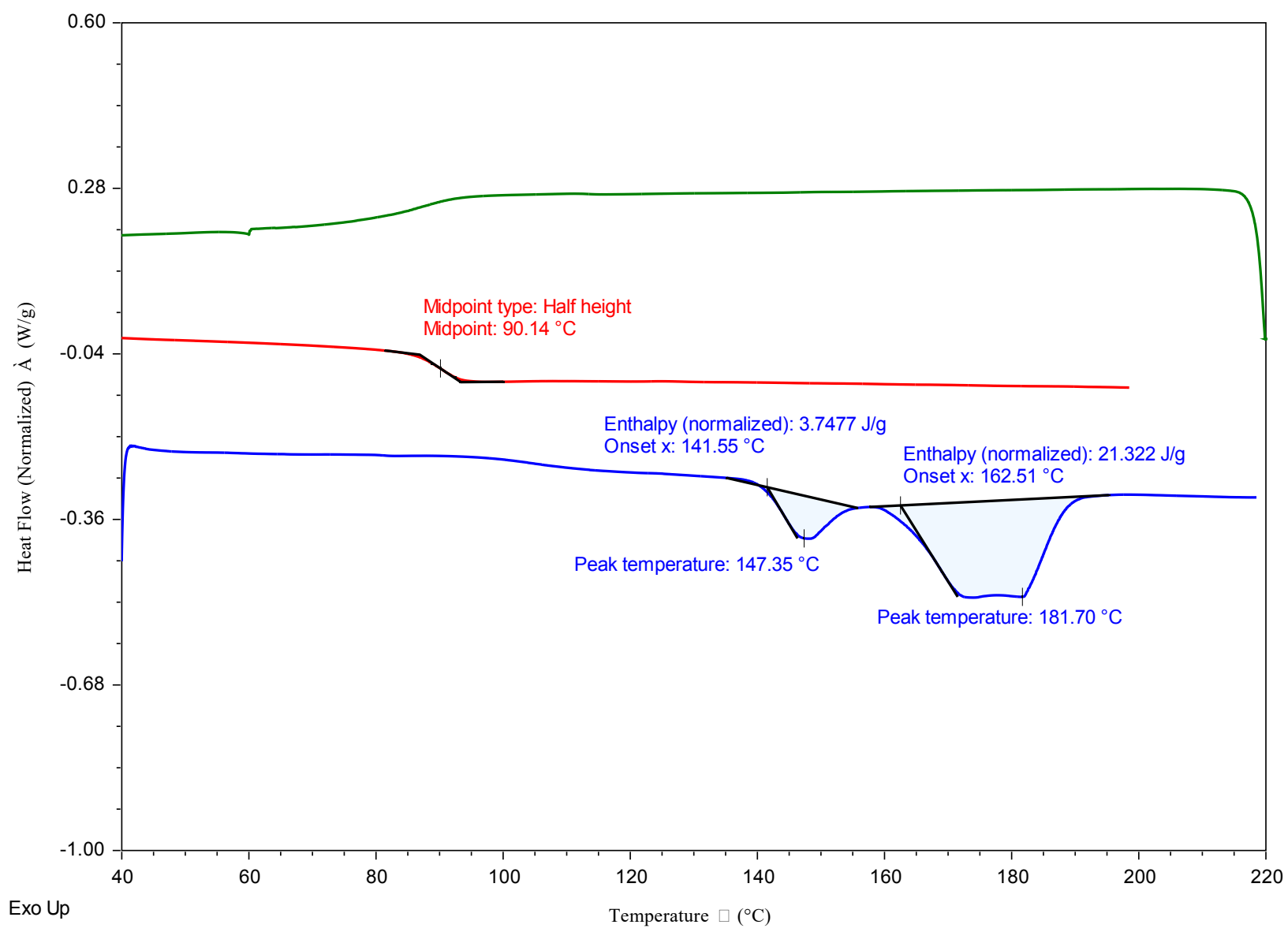

Figure S45. DSC curves of poly $(\mathrm{D}-$ manOCA $)\left(M_{\mathrm{n}}=5400, Ð=1.11\right)$ produced via the ROP of $\mathrm{D}^{-m a n O C A}$ using complex 1 as a catalyst (Table S1, entry 14), $T_{\mathrm{m} 1}=147{ }^{\circ} \mathrm{C}$ (first heating scan); $T_{\mathrm{m} 2}$ $=182^{\circ} \mathrm{C}$ (first heating scan); $T_{\mathrm{g}}=90^{\circ} \mathrm{C}$ (second heating scan). (Heating and cooling rate $10{ }^{\circ} \mathrm{C} / \mathrm{min}$, first heating: blue line, first cooling: green line, second heating: red line). 


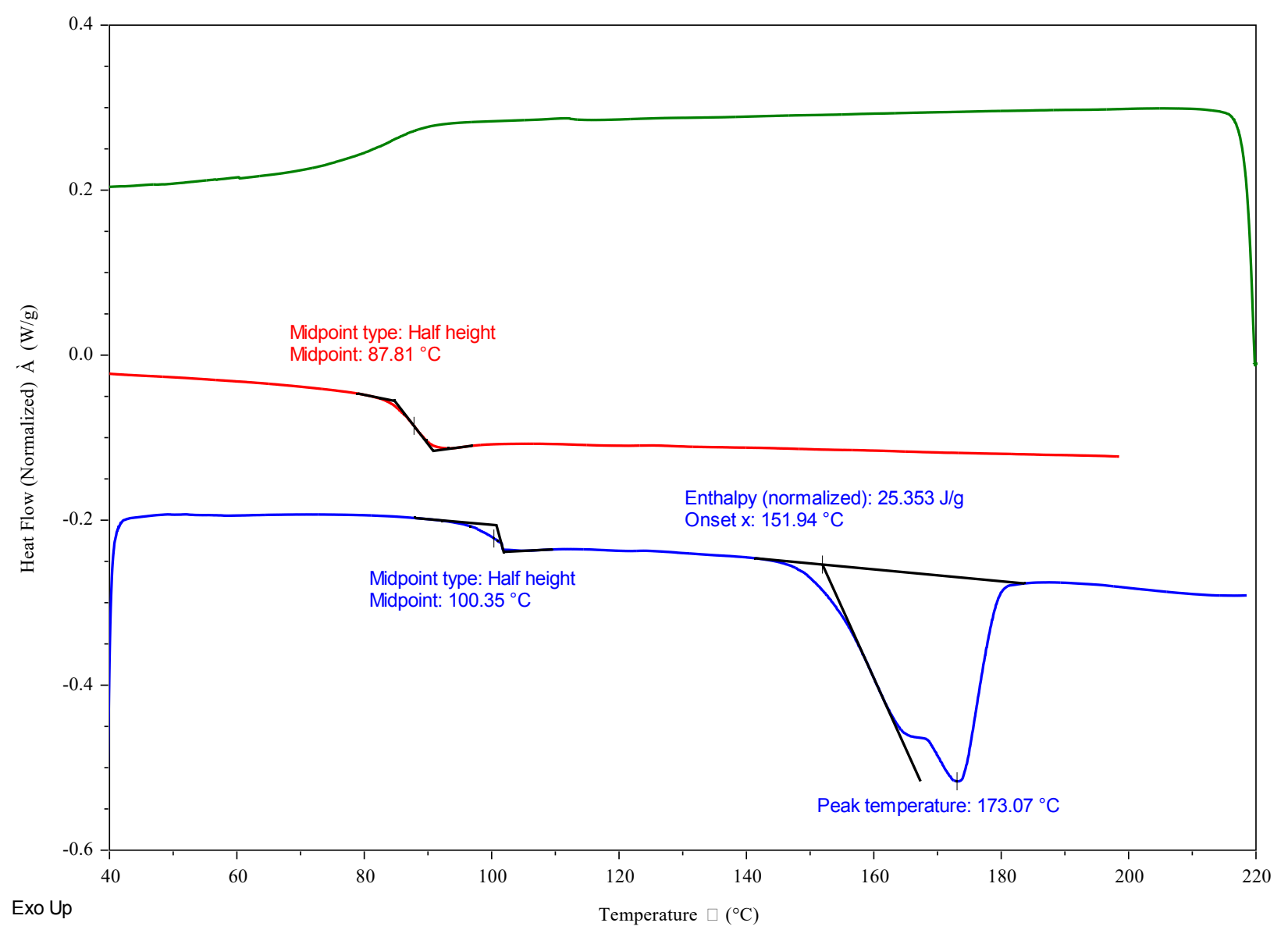

Figure S46. DSC curves of poly $(\operatorname{manOCA})\left(M_{\mathrm{n}}=5400, Ð=1.10\right)$ produced via mixing $\operatorname{poly}\left({ }_{\mathrm{L}}\right.$-manOCA $)\left(M_{\mathrm{n}}=5300, Ð=1.10\right.$, Table 1 ,entry 1$)$ and poly $(\mathrm{D}$-manOCA $)\left(M_{\mathrm{n}}=5400, Ð=\right.$ 1.11, Table S1,entry 14), $T_{\mathrm{m}}=173{ }^{\circ} \mathrm{C}$ (first heating scan), $T_{\mathrm{g}}=88^{\circ} \mathrm{C}($ second heating scan) at a $1: 1$ ratio. (Heating and cooling rate $10{ }^{\circ} \mathrm{C} / \mathrm{min}$, first heating: blue line, first cooling: green line, second heating: red line). 


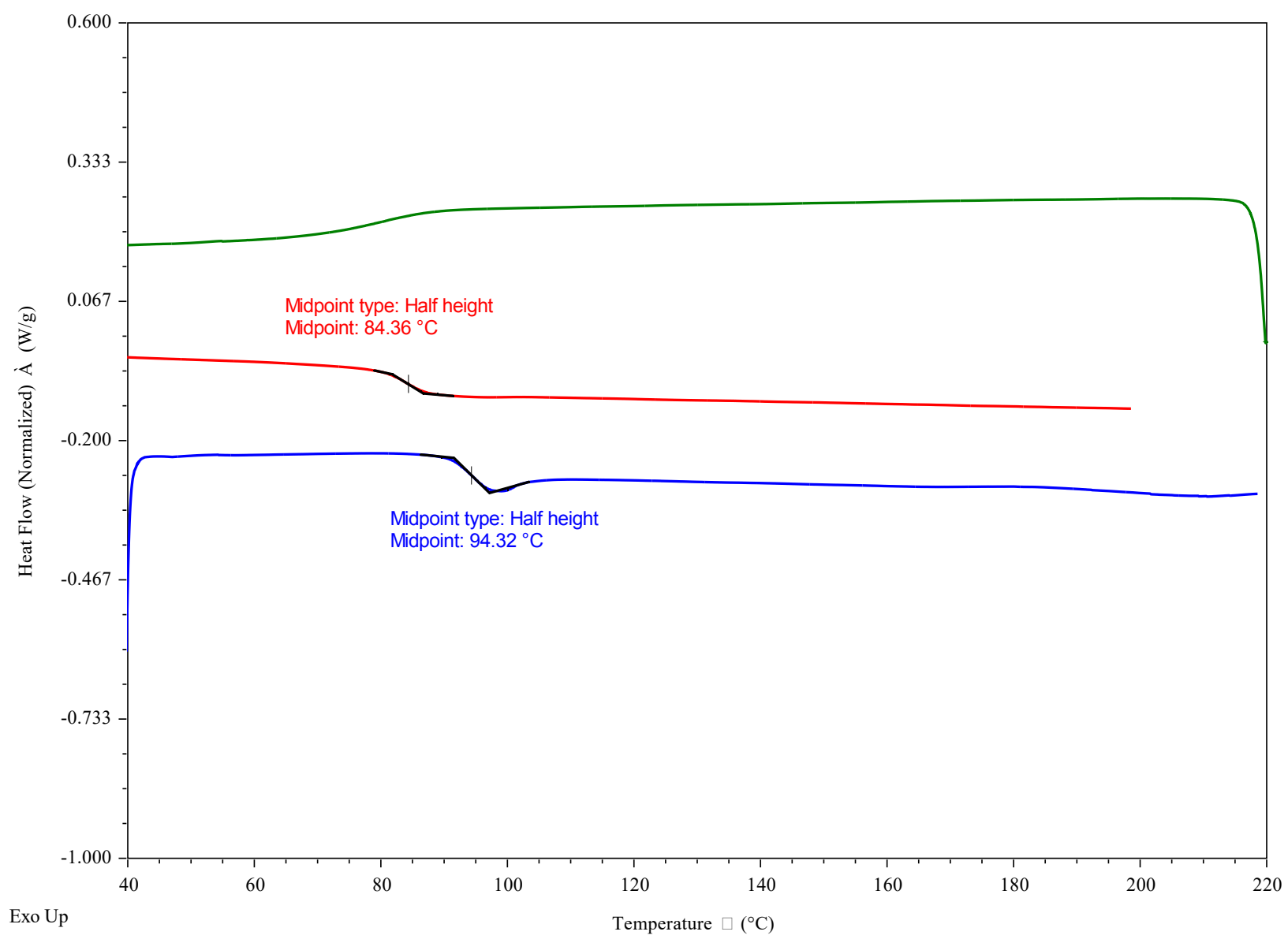

Figure S47. DSC curves of poly $(r a c-m a n O C A)\left(P_{\mathrm{m}}=0.6\right)$ produced via the ROP of rac-manOCA using complex 1 as a catalyst (Table 2 , entry 1 ), no $T_{\mathrm{m}}$ in first heating scan, $T_{\mathrm{g}}=84{ }^{\circ} \mathrm{C}$ (in second heating scan). (Heating and cooling rate $10^{\circ} \mathrm{C} / \mathrm{min}$, first heating: blue line, first cooling: green line, second heating: red line). 


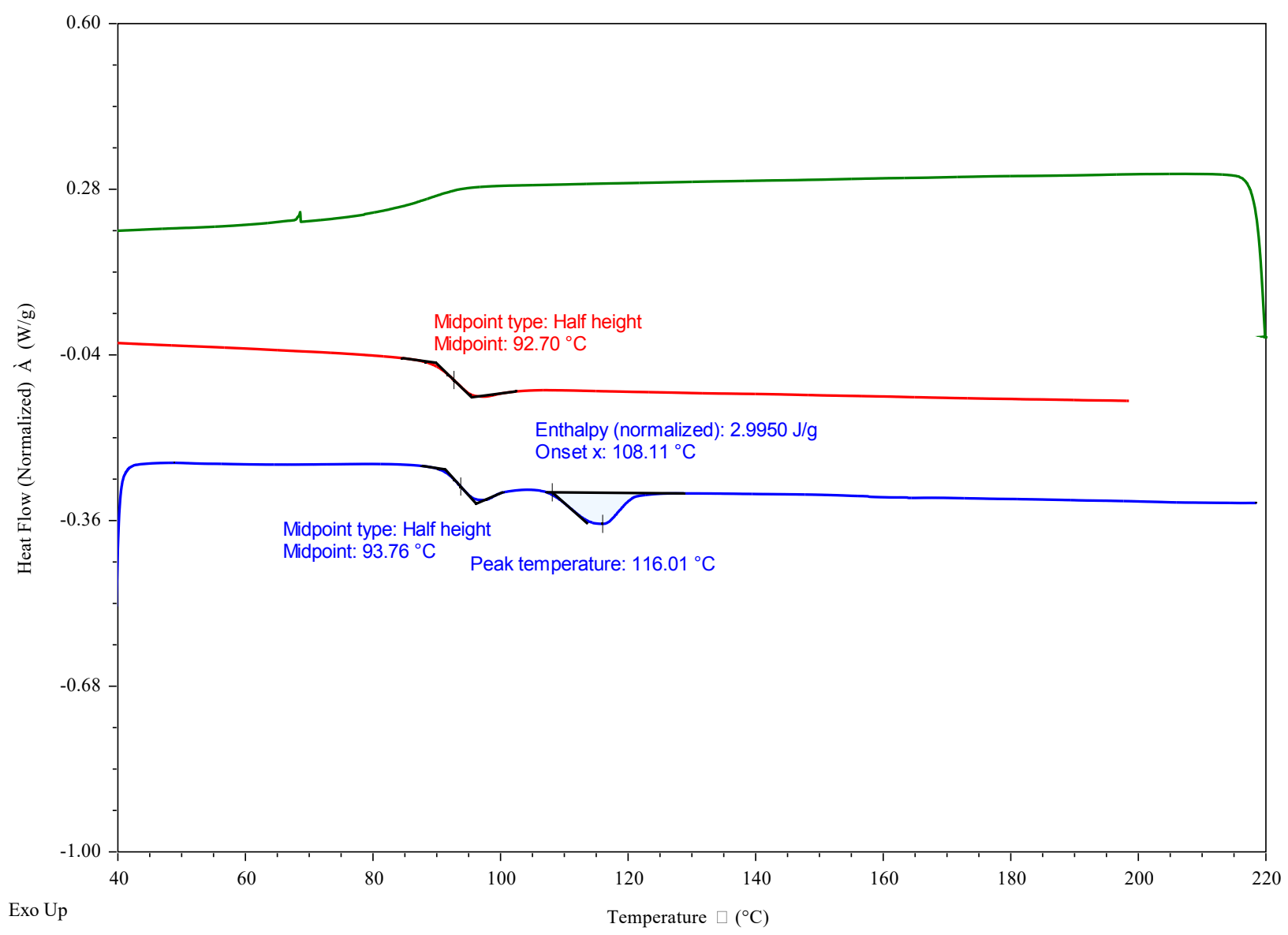

Figure S48. DSC curves of poly $(r a c-m a n O C A)\left(P_{\mathrm{m}}=0.92\right)$ produced via the ROP of $r a c-m a n \mathrm{OCA}$ using complex 1 as a catalyst (Table 2, entry 4), $T_{\mathrm{m}}=116{ }^{\circ} \mathrm{C}$ (in first heating scan), $T_{\mathrm{g}}=93{ }^{\circ} \mathrm{C}$ (in second heating scan). (Heating and cooling rate $10{ }^{\circ} \mathrm{C} / \mathrm{min}$, first heating: blue line, first cooling: green line, second heating: red line). 


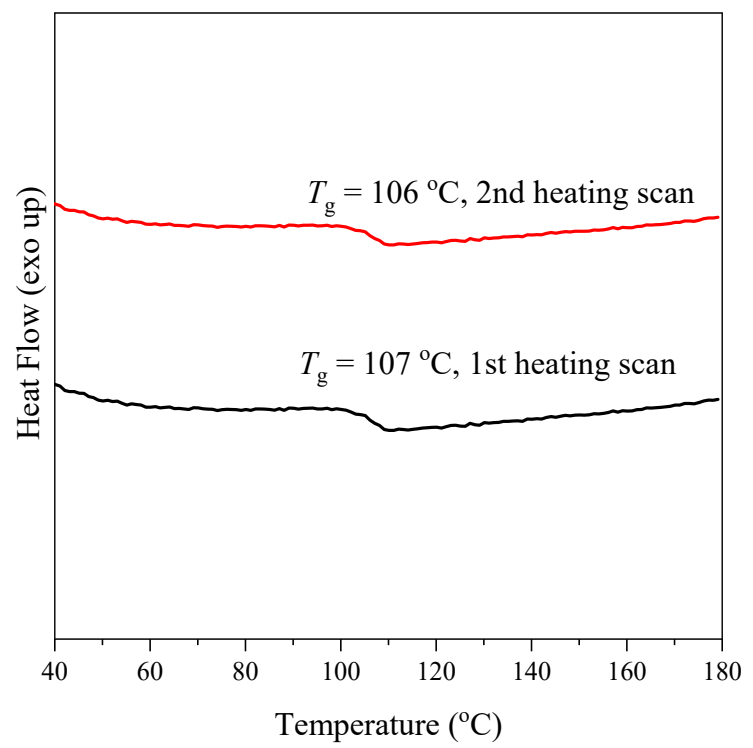

Figure S49. DSC curves of poly $\left({ }_{L}-\right.$ manOCA $)\left(M_{\mathrm{n}}=24200, \emptyset=1.13\right.$; Table 1, entry 6$)$ (the sample was recrystallized from n-hexane and $\mathrm{CH}_{2} \mathrm{Cl}_{2}$, then the samples were vacuumed at $130{ }^{\circ} \mathrm{C}$ for $3 \mathrm{~h}$ before program heating to eliminate the effects of solvents).

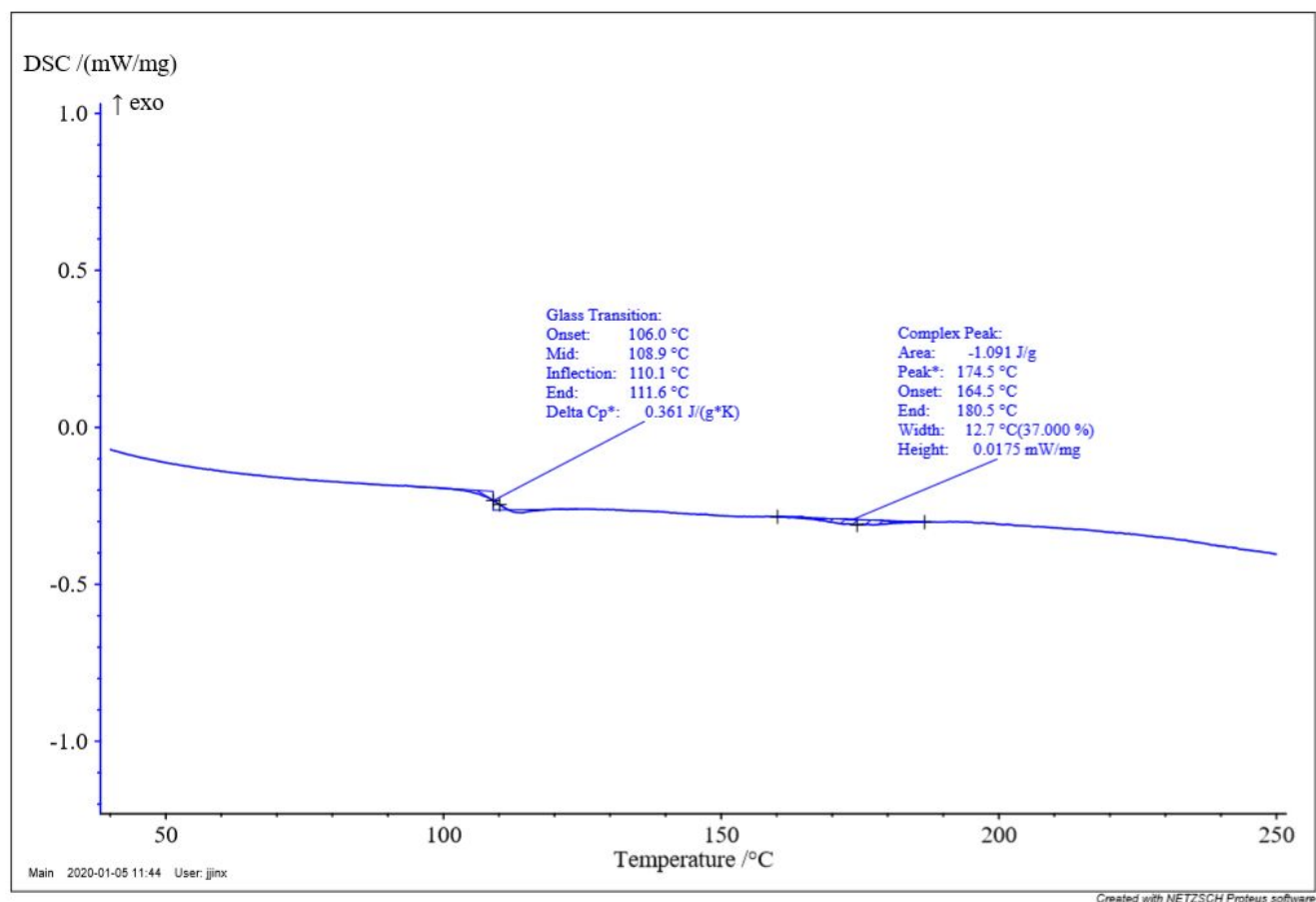

Figure S50. DSC curves of poly $\left({ }_{\mathrm{L}}-\operatorname{manOCA}\right)\left(M_{n}=24200 \mathrm{~kg} / \mathrm{mol}\right)$ produced via the ROP of $\mathrm{L}^{-}$-manOCA using complex 1 as a catalyst (Table 1, entry 6), $T_{\mathrm{g}}=109{ }^{\circ} \mathrm{C}, T_{\mathrm{m}}=174.5{ }^{\circ} \mathrm{C}$, (no solvent-induced crystallization, the samples quickly precipitate from the solvent by adding a large amount of methanol to the polymer DCM solution, then the samples were vacuumed at $130{ }^{\circ} \mathrm{C}$ for 3 $\mathrm{h}$ before program heating to eliminate the effects of solvents). 


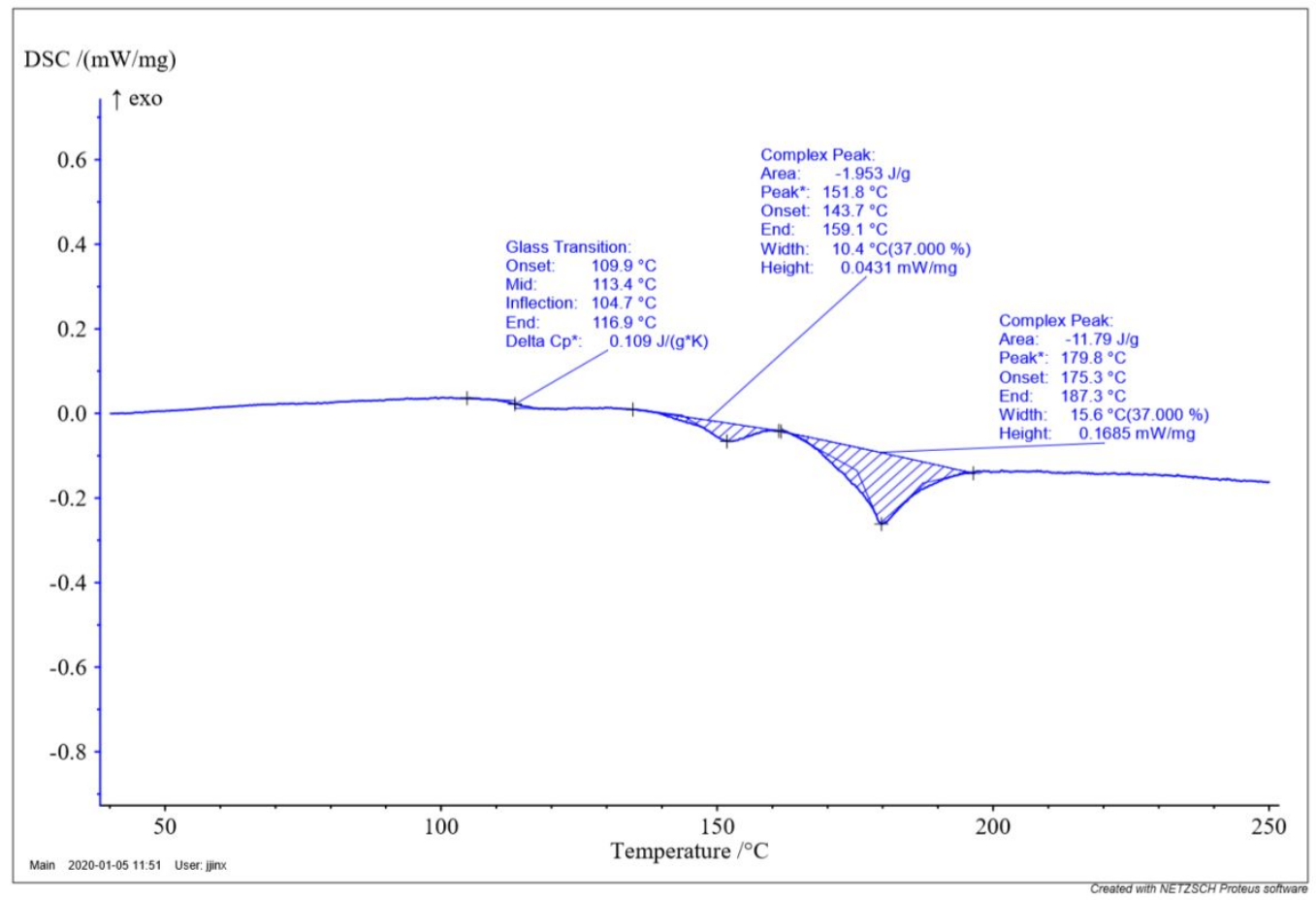

Figure S51. DSC curves of poly(L-manOCA) $\left(M_{n}=24200 \mathrm{~kg} / \mathrm{mol}\right)$ produced via the ROP of ${ }_{\mathrm{L}}$-manOCA using complex 1 as a catalyst (Table 1 , entry 6$), T_{\mathrm{m} 1}=152{ }^{\circ} \mathrm{C}, T_{\mathrm{m} 2}=180{ }^{\circ} \mathrm{C}, T_{\mathrm{g}}=113$ ${ }^{\circ} \mathrm{C}$. (after solvent-induced crystallization, the samples were vacuumed at $130{ }^{\circ} \mathrm{C}$ for $3 \mathrm{~h}$ before program heating to eliminate the effects of solvents). 


\section{Wide-angle X-ray scattering (WAXS) spectra}
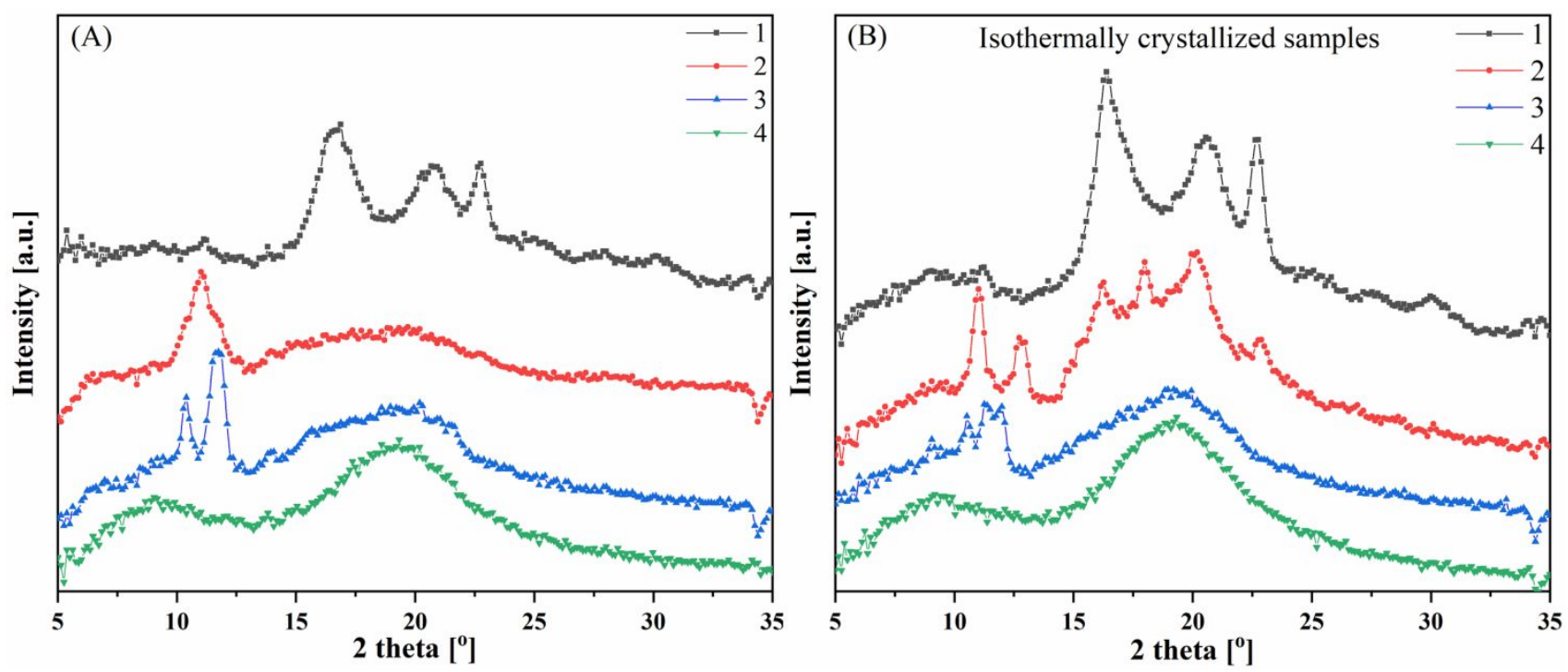

Figure S52. Wide-angle X-ray scattering (WAXS) spectra of 1: poly $\left({ }_{L}-m a n O C A\right) ~\left(M_{\mathrm{n}}=5.3 \mathrm{~kg} / \mathrm{mol}\right.$, $Ð=1.10$; Table 1 , entry 1$) ; 2$ : poly $\left({ }_{L}-m a n O C A\right)$ and poly $\left(D^{-m a n O C A}\right)$ at $1: 1$ ratio $\left(M_{\mathrm{n}}=5.3 \mathrm{~kg} / \mathrm{mol}\right.$, $Ð=1.10) ; 3$ : poly (rac-manOCA) $\left(P_{\mathrm{m}}=0.92, M_{\mathrm{n}}=5.3 \mathrm{~kg} / \mathrm{mol}, \emptyset=1.12\right.$; Table 2, entry 4); 4: poly (rac-manOCA) $\left(P_{\mathrm{m}}=0.6, M_{\mathrm{n}}=5.4 \mathrm{~kg} / \mathrm{mol}, \emptyset=1.10\right.$; Table 2, entry 1), (A): These samples were prepared by the following method: about $40 \mathrm{mg}$ poly $(\operatorname{manOCA})\left(M_{\mathrm{n}} \approx 5300\right)$ was dissolved in 2 $\mathrm{mL}$ of DCM, stirred slowly for $6 \mathrm{~h}$, then $4 \mathrm{~mL}$ of methanol was added dropwise, the solution formed two phases. After stirring for several hours, a white powder was precipitated. After Stop stirring for 30 minutes, the filtrate was poured out and the sample was vacuum dried. (B) The obtained polymers samples from (A) were vacuum dried under a $\mathrm{N}_{2}$ atmosphere at a specific temperature for 3 hours (reference to dsc sample preparation method). 


\section{In-suit WAXS spectrum}

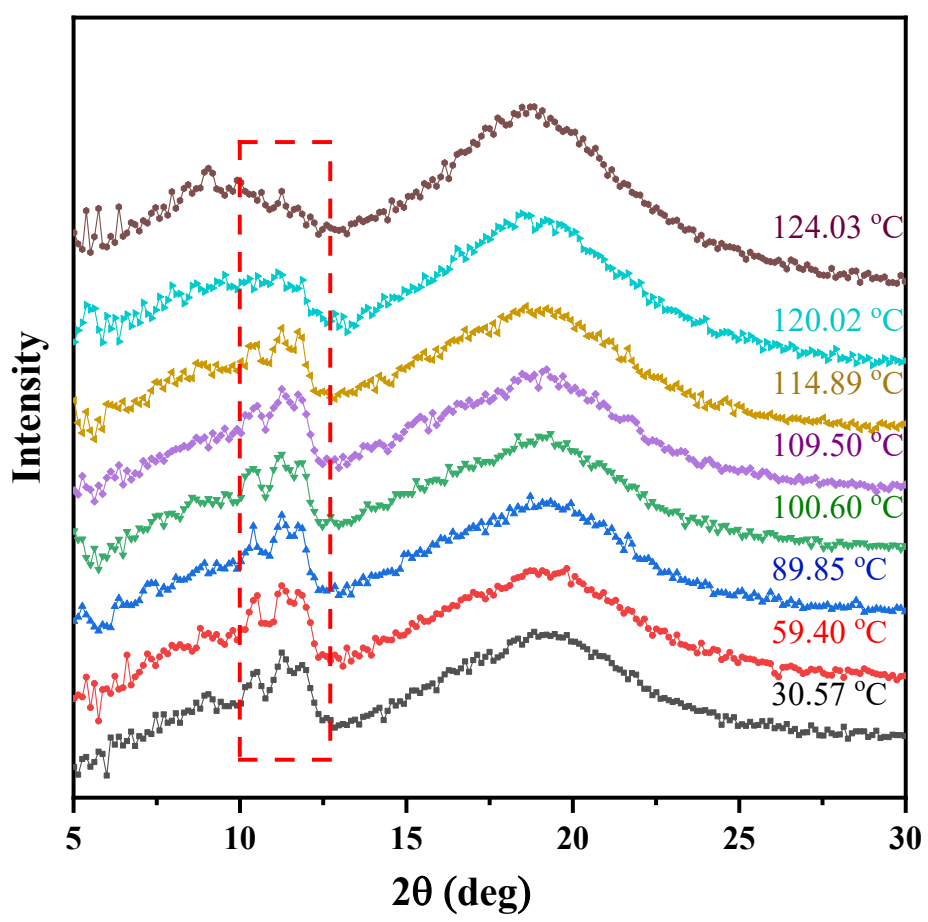

Figure S53. In-suit WAXS curves of the poly(rac-manOCA) $\left(P_{\mathrm{m}}=0.92\right)$ as a function of sample temperature (Heating rate $10{ }^{\circ} \mathrm{C} \mathrm{min}-1$, each temperature was exposed for 6 seconds). The WAXS diffraction signal $\left(2 \theta=10.52,11.26\right.$, and $\left.11.87^{\circ}\right)$ disappeared at about $120{ }^{\circ} \mathrm{C}$, which matched the DSC heating process curve well (Figure S6A). The second peak of the DSC curve was judged to be the melting peak of the sample.

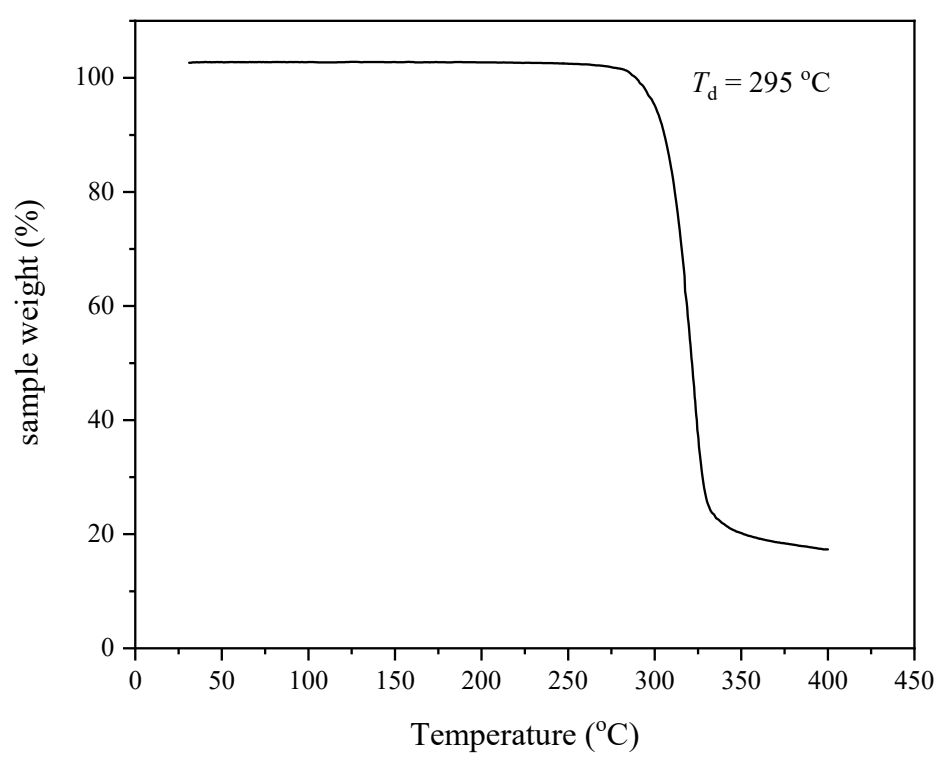

Figure S54: Thermal stability of poly $\left(\mathrm{L}_{\mathrm{L}}-\operatorname{manCOA}\right)\left(M_{\mathrm{n}}=24200\right)$ using complex 1 as a catalyst (Table 1 , entry 6$) . T_{\mathrm{d}}=295^{\circ} \mathrm{C}(5 \%$ weight loss $)$. 


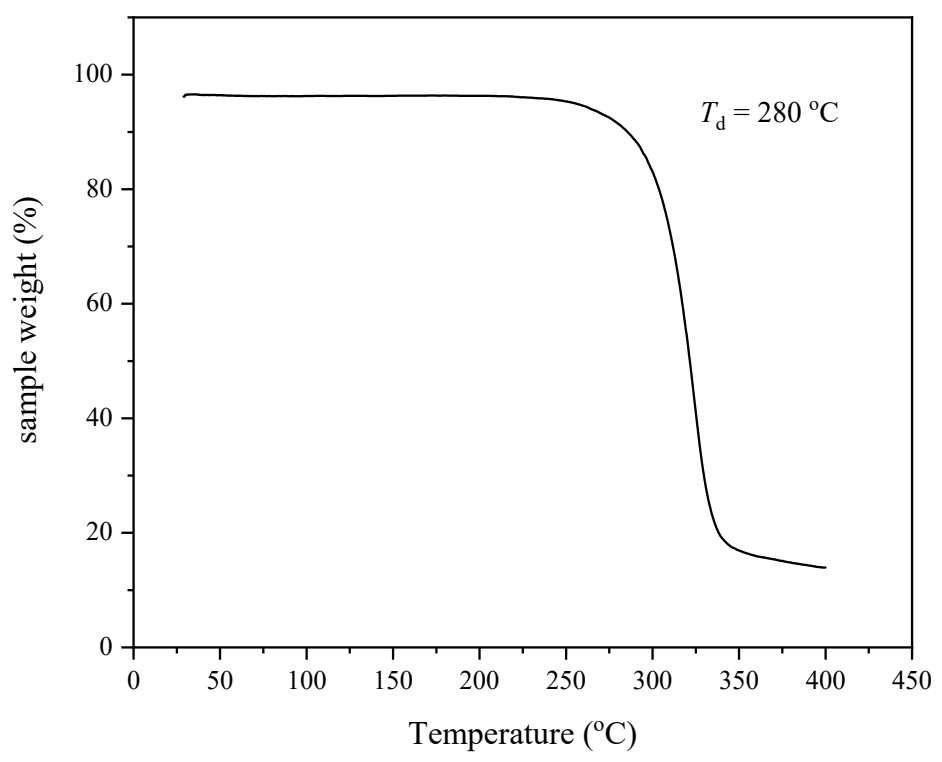

Figure S55: Thermal stability of poly $(\mathrm{rac}-\operatorname{manCOA})\left(P_{\mathrm{m}}=0.60, M_{\mathrm{n}}=5300\right)$ using complex 1 as a catalyst (Table 2 , entry 1$) . T_{\mathrm{d}}=280{ }^{\circ} \mathrm{C}(5 \%$ weight loss $)$.

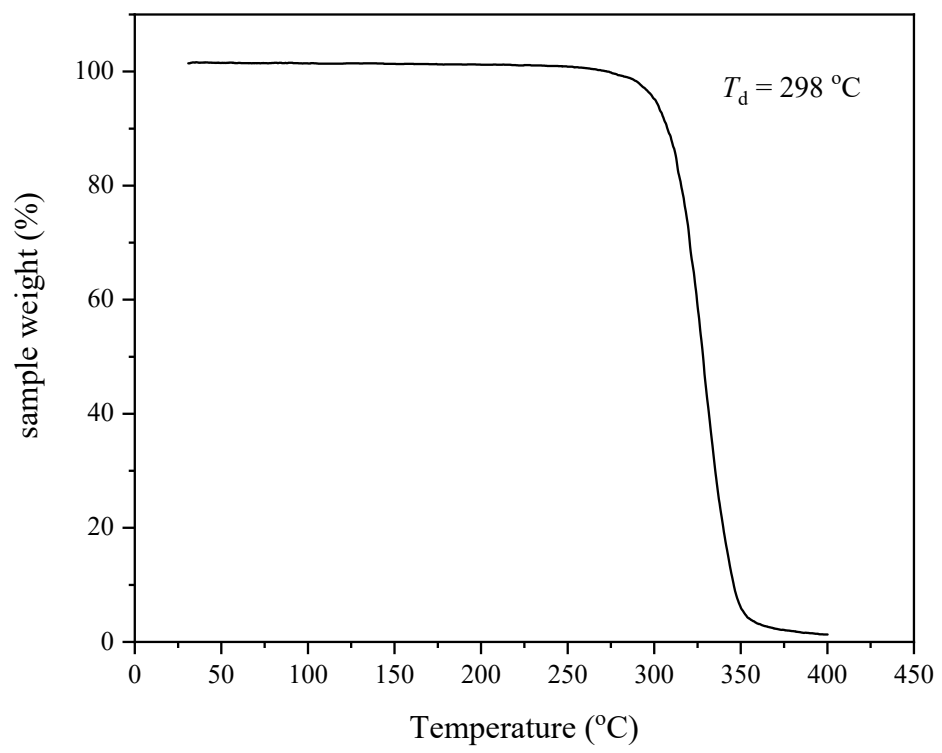

Figure S56: Thermal stability of poly $(\mathrm{rac}-\operatorname{manCOA})\left(P_{\mathrm{m}}=0.92, M_{\mathrm{n}}=5300\right)$ using complex 1 as a catalyst (Table 2 , entry 4$) . T_{\mathrm{d}}=298{ }^{\circ} \mathrm{C}(5 \%$ weight loss $)$. 


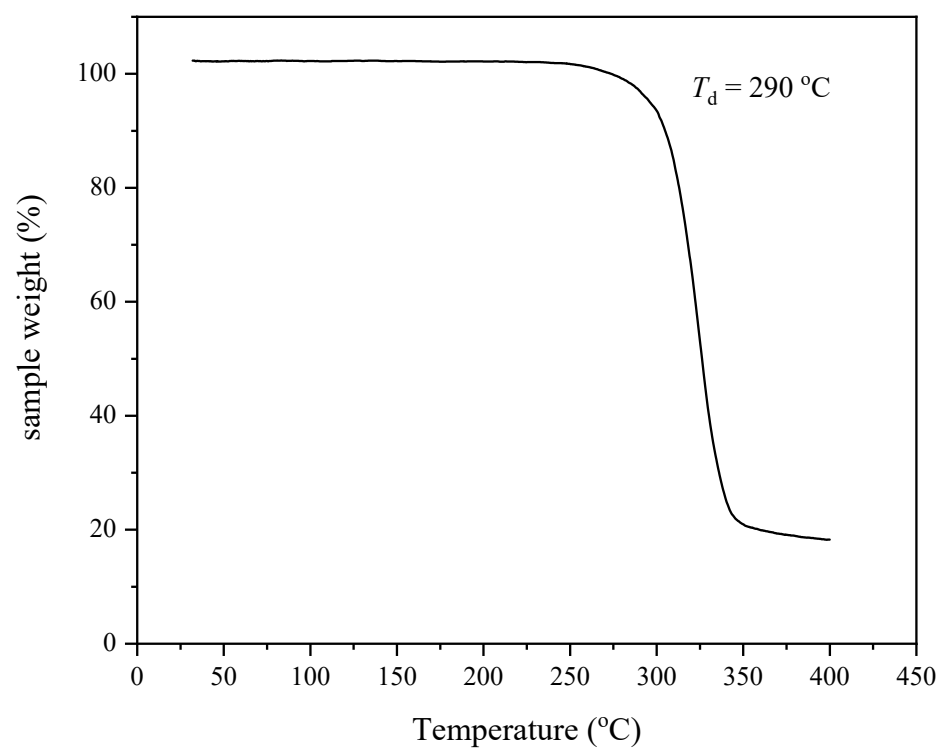

Figure S57: Thermal stability of poly $\left(\mathrm{L}_{-}-\operatorname{manCOA}\right)\left(M_{\mathrm{n}}=5300\right)$ using complex 1 as a catalyst (Table 1, entry 1) $T_{\mathrm{d}}=290{ }^{\circ} \mathrm{C}$ (5\% weight loss). 

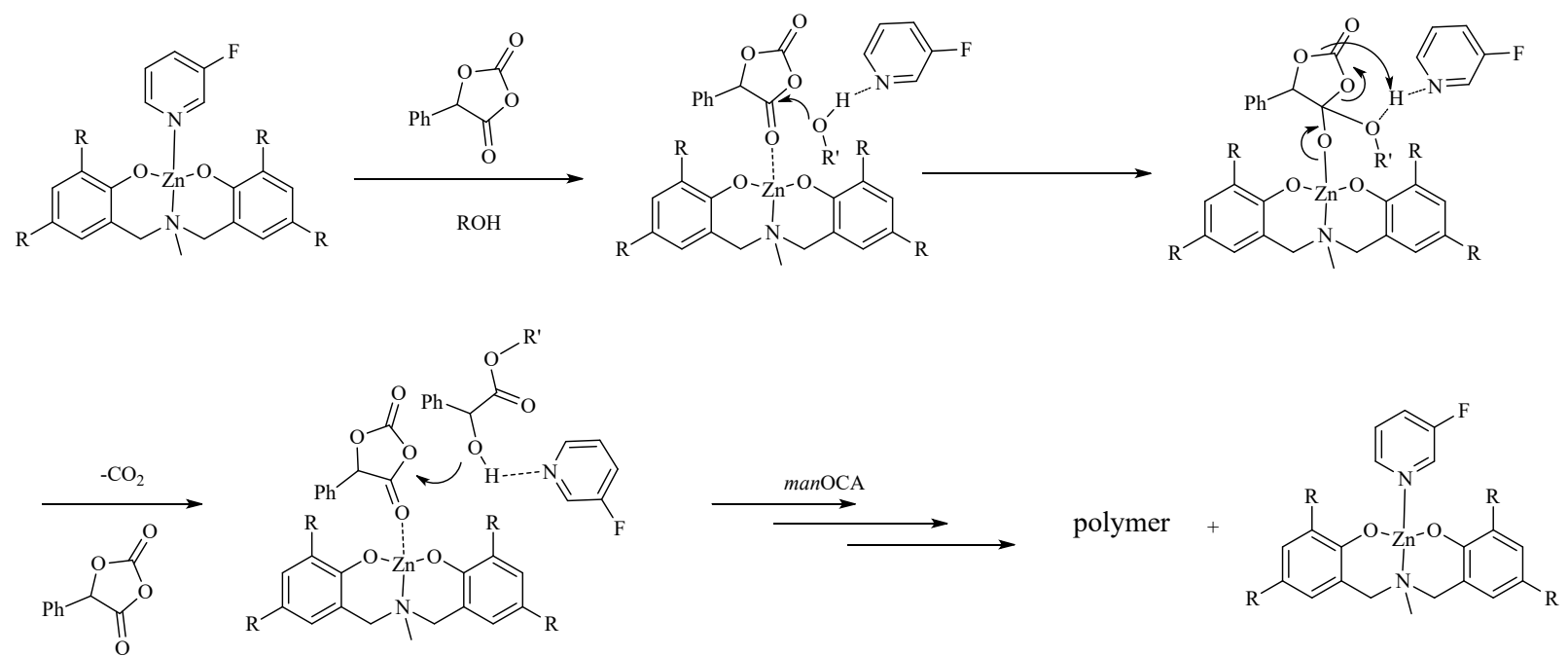

Scheme S1. proposed mechanism for the ROP of manOCA using Lewis pairs of zinc complex + 3-F-Py as a catalyst. 


\section{REFERENCES}

1. Darensbourg, D. J.; Holtcamp, M. W.; Struck, G. E.; Zimmer, M. S.; Niezgoda, S. A.; Rainey, P.;

Robertson, J. B.; Draper, J. D.; Reibenspies, J. H., Catalytic Activity of a Series of $\mathrm{Zn}(\mathrm{II})$ Phenoxides for the Copolymerization of Epoxides and Carbon Dioxide. J. Am. Chem. Soc. 1999, $121,107-116$.

2. Lee, D.-Y.; Hartwig, J. F., Zinc Trimethylsilylamide as a Mild Ammonia Equivalent and Base for the Amination of Aryl Halides and Triflates. Org. Lett. 2005, 7, 1169-1172.

3. Tang, L.; Deng, L., Dynamic Kinetic Resolution via Dual-Function Catalysis of Modified Cinchona Alkaloids: Asymmetric Synthesis of $\alpha$-Hydroxy Carboxylic Acids. J. Am. Chem. Soc. 2002, 124, 2870-2871.

4. Sheldrick, G. M., Shelxl-97. University of Göttingen: Göttingen, Germany: 1996.

5. Silvernail, C. M.; Yao, L. J.; Hill, L. M. R.; Hillmyer, M. A.; Tolman, W. B., Structural and Mechanistic Studies of Bis(phenolato)amine Zinc(II) Catalysts for the Polymerization of ع-Caprolactone. Inorg. Chem. 2007, 46, 6565-6574.

6. Kuchuk, E. A.; Zaitsev, K. V.; Mamedova, F. A.; Churakov, A. V.; Zaitseva, G. S.; Lemenovsky, D. A.; Karlov, S. S., Synthesis, Structure, and Catalytic Activity of New Aluminum and Titanium Complexes Based on Aminobisphenolate Ligands Containing Bulky Substituents. Russ. Chem. Bull. 2016, 65, 1743-1749.

7. Belostotskaya, I. S.; Komissarova, N. L.; Prokof'eva, T. I.; Kurkovskaya, L. N.; Vol'eva, V. B., New Opportunities for Duff Reaction. Russ. J. Org. Chem. 2005, 41, 703-706. 
8. Wang, D.; Lindeman, S. V.; Fiedler, A. T., Synthesis of Homo- and Heterobimetallic NiII-MII $(\mathrm{M}=\mathrm{Fe}, \mathrm{Co}, \mathrm{Ni}, \mathrm{Zn})$ Complexes Based on an Unsymmetric Ligand Framework: Structures, Spectroscopic Features, and Redox Properties. Inorg. Chim. Acta 2014, 421, 559-567. 